\title{
LA-3067-MS, Rev.
}

Informal Report

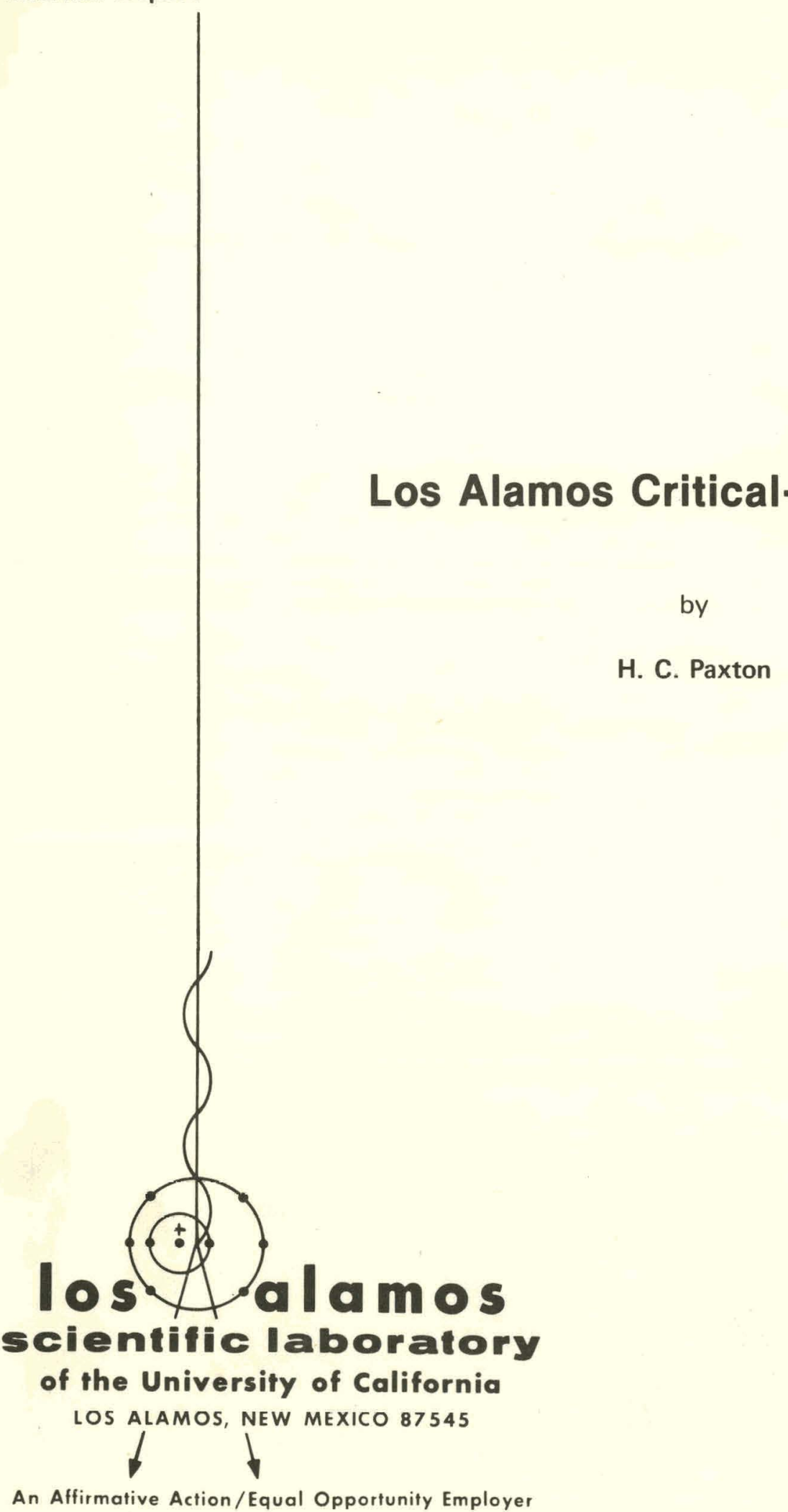

An Affirmative Action/Equal Opportunity Employer
UC-46

Reporting Date: November 1975 Issued: December 1975 


\section{DISCLAIMER}

This report was prepared as an account of work sponsored by an agency of the United States Government. Neither the United States Government nor any agency Thereof, nor any of their employees, makes any warranty, express or implied, or assumes any legal liability or responsibility for the accuracy, completeness, or usefulness of any information, apparatus, product, or process disclosed, or represents that its use would not infringe privately owned rights. Reference herein to any specific commercial product, process, or service by trade name, trademark, manufacturer, or otherwise does not necessarily constitute or imply its endorsement, recommendation, or favoring by the United States Government or any agency thereof. The views and opinions of authors expressed herein do not necessarily state or reflect those of the United States Government or any agency thereof. 


\section{DISCLAIMER}

Portions of this document may be illegible in electronic image products. Images are produced from the best available original document. 
In the interest of prompt distribution, this report was not edited by the Technical Information staff.

Printerl in the United States of America. Available from

National Technical Information Service

U.S. Department of Commerce

5285 Port Royal Ruad

Springfield, VA 2215

Price: Printed Copy $\$ 5.50$ Microfiche $\$ 2.25$

This report was prepared as an account of work sponsored

bs the United States Government. Neither the United States
nor the United States Energy Research and Development Ad-

nor the United States Energy Research and Development Ad-
ministration. nor any of their employees, nor any of their con-

tractors, subcontractors, or their employees. makes any

responsibility for the accuracy, completeness, or usefulness of

any information, apparatus, product, or process disclosed, or

represents that its use would not infringe privately owned 


\section{PAGES $\mathrm{i}$ to $\mathrm{ii}$ WERE INTENTIONALLY LEFT BLANK}




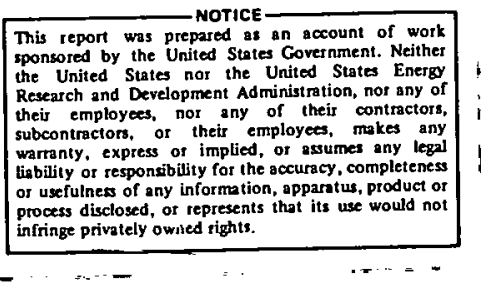

TABLE OF CONTENTS

Page

ABSTRACT •. . . . . . . . . . . . . . . . 1



\section{TABLES}

IAl Highly Enriched U Metal, Unreflected. . . . 4

IA2 U(93.4) - U(N) Metal Cylinders,

Unreflected . . . . . . . . . . . . . 5

IA3 $U(93.3)-U(N)$ Metal Cylinders,

15.00" Diameter, Unreflected. . . . . . . 6

IA4 U(93.3) - U(N) Metal Cylinders, 21" Diameter, Unreflected . . . . . . . . . . . . 7

IB1 Enriched U Metal Sphere or Pseudosphere,

$\mathrm{U}(\mathrm{N})$ Reflector. . . . . . . . . . . 8

IB2 Enriched U Metal Cylinder, Pseudocylinder

or Parallelepiped, U(N) Reflector . . . . 9

IB3 Miscellaneous Enriched U Meta1,

U(N) Reflector. . . . . . . . . . . 10

ICl Highly Enriched U Metal, Reflector of

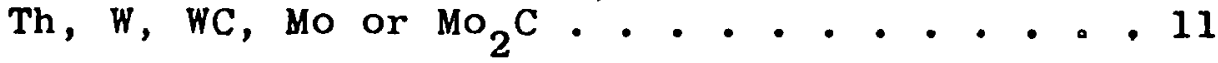

IC2 Highly Enriched U Metal, Reflector of

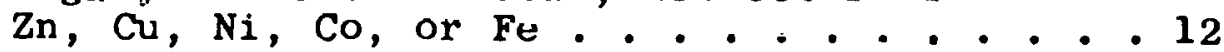

IC3 Highly Enriched U Metal, Reflector of

$\mathrm{Ti}, \mathrm{Al}, \mathrm{Al}_{2} \mathrm{O}_{3}, \mathrm{Mg}, \mathrm{Be}$, or $\mathrm{BeO} \cdot . \cdot . \cdot . \cdot .13$

IC4a Highly Enrlched U Metal, Complete

Graphite Reflector. . ...... . . . . 14 
TABLES (Continued)

Page

IC4b Highly Enriched U Metal, Partial

Graphite Reflector. . . . . . . . . . . 15

IC5 Highly Enriched U Metal, Reflector of



IC6a Highly Enriched U Metal, Complete

Reflector of $\mathrm{H}_{2} \mathrm{O}$ or Polyethylene... . . . . 17

IC6b Highly Enriched U Metal, Complete

Reflector of Paraffin........ . . . . 18

IC6c Highly Enriched U Metal, Partial Reflector

of $\mathrm{H}_{2} \mathrm{O}$, Polyethylene, Lucite, or Paraffin •. 19

IC7 Highly Enriched U, Mixed Reflector. . . . . 20

IIAI U(93.3) Metal Cylinders Diluted with

$\mathrm{Fe}, \mathrm{Ni}, \mathrm{Cu}$, or $\mathrm{Zn}, 15^{\prime \prime}$ Diameter, Unreflected. . 21

IIA2 U(93.3) Metal Cylinders Diluted with

Mo, Ta, or W, 15" Diameter, Unreflected . . . 22

IIA3 U(93.3) Metal Cylinders Diluted with

$\mathrm{Al}, \mathrm{Zr}$, or Hf, 15" Diameter, Unreflected. . . 23

IIBI U(93.3) - Graphite Cylinders, Unreflected . . 24

I IB2a Graphite-Moderated Highly-Enriched U,

Reflector of Graphite . . . . . . . . . 25

I IB2b Graphite-Moderated Highly-Enriched U,

Reflector of Be (Plus some Graphite). . . . . 26

IICl Lucite Moderated U(93.16), Unreflected. • . . 27

IIC2a Lucite Moderated U(93.16), $\geq 6^{\prime \prime}$ Thick

Lucite Reflector。. . . . . . . . . . 28

I IC2b Lucite Moderated U(93.16) Slabs, 6" Thick

Lucite Reflectors on Two Large Surfaces Only. . 29

IIC3 Polyethylene Moderated U(93.15) Parallel-

epipeds, Beryllium Reflector. 
IID1 Lucite-Graphite Moderated U(93.16),

Unreflected. . . . . . . . . . . . 31

IID2a Lucite-Graphite Moderated U(93.16),

Lucite Reflected . . . . . . . . . . . 32

IID2b Lucite-Graphite Moderated U(93.16) Slabs, 6" Thick Lucite Reflectors on Two

Large Surfaces Only. . . . . . . . . . . 33

IIEl Diffuse U(93.1) Reflected by Thick $\mathrm{D}_{2} \mathrm{O}$

or Be (Cavity Assemblies). . . . . . . . . 34

IIE2 U(93.65) $\mathrm{O}_{2} \mathrm{~F}_{2}-\mathrm{D}_{2} \mathrm{O}$ Solutions, Bare, $\mathrm{D}_{2} \mathrm{O}$ or Graphite Reflected . . . . . . . . . . 35

IIFla $\mathrm{U}(14.67) \mathrm{O}_{2} \mathrm{SO}_{4}-\mathrm{H}_{2} \mathrm{O}$ Solution, Sphere . . . . . 36

IIFlb U(93.5)-Phosphate Aqueous Solution

Cylinders, 3.0" Thick Fe Reflector . . . . . 37

IF2 Enriched-Uranium Hydride Composition . . • . 38

IIF3 Lattices of $\mathrm{U}(94)$ Metal Units, $\mathrm{H}_{2} \mathrm{O}$

Moderated, $\mathrm{H}_{2} \mathrm{O}$ Reflected . . . . . . . 39

IIIAl Plutonium-Metal spheres. . . . . . . . . . 40

IIIA.2 Plutonium in Metal-Polyethylene Reflector,

Spherical Geometry ............. . 4l

IIIA3 plutonium-Metal Cylinders. . . . . . . . . . 42

IIIBI Diluted Pu Cylinders, 6.0" Diameter, Unreflected. . . . . . . . . . . . . 43

IIIB2 Diluted Pu Cylinders, 6.0" Diameter, $2.0 "$ Thick U( 0.3) Reflector . . . . . . 44

I I IB3 Diluted Pu Cylinders, 6.0" Diameter, 4.5" Thick U( 0.3) Reflector . . . . . . 45

IIIB4 Diluted Pu Cylinders, 6.0" Diameter, $7.5 "$ Thick U(.u.3) Reflector . . . . . . 46

IIIB5 Diluted Pu Cylinders, 6.0" Diameter, 2.0" Thick Th Reflector. . . . . . . . . 47

IIIB6 Diluted Pu Cylinders, 6.0" Diameter, 4.5" Thick Th Reflector. . . . . . . . . 48 


\section{TABLES (Continued)}

Page

IIIB7 Diluted Pu Cylinders, 6.0 Diameter

7.5" Thick Th Reflector. . . . . . . . 49

IV U-233 Metal Spheres. . . . . . . . . 50

VA Pu or U-233 Metal Spheres Within

U( 93) Metal Spheres........... 51

vB Pu Metal Cylinder Within U(93.2) Metal

Cylinder, Thick U(N) Reflector . . . . . 52

REFERENCES. . . . . . . . . . . . . . . . 53 


\title{
LOS ALAMOS CRITICAL-MASS DATA
}

\author{
by \\ H.C. Paxton
}

\begin{abstract}
The original version of this report tabulates critical masses of simple systems, which have been measured through the year 1963. This revision adds data through October 1975, and modifies some of the old critical specifications that have been reevaluated. The old format and symbolism are retained to simplify reproduction.
\end{abstract}




\section{INTRODUCTION}

Numerous Los Alamos critical mass data have been published only as points on curves, frequently after adjustment to "standard" conditions (e.g., to uniform values of $\mathrm{v}^{235}$ enrichment and density), and usually without indication of reliability. Under these conditions, original data tend to become lost. It is the purpose of this compilation to retrieve original critical masses and to give some means of judging the quality of measurements.

Indexes of accuracy are probable error, if it has been estimated, the maximum mass of fissile material used in the measurement, or the maximum central-source neutron multiplication attained. For nonhydrogenous systems a multiplication of 10 usually corresponds to a core mass that is $70 \%$ to $80 \%$ of critical, 20 corresponds to $85 \%$ to $90 \%$ of the critical mass, 50 corresponds to $93 \%$ to $97 \%$, and 100 corresponds to $96-1 / 2 \%$ to $98-1 / 2 \%$. Generally, the probable error in critical mass is about one-quarter of the difference between the critical mass value and the maximum mass employed. This estimate may be valid down to an indicated probable error of $1 \%$ to $2 \%$, beyond which the probable error is usually controlled by the precision with which the composition and geometry of the system can be described. Maximum multiplication is not a reliable index of accuracy for hydrogen-moderated assemblies because of the severe influence of neutron-spectral distortion.

Not included in this compilation are numerous critical assemblies such as reactor mockups, which cannot be described adequately by simple entries in tables. Also omitted are a few critical mass estimates for which the maximum mass used 
was less than three-quarters of the critical value.

The following symbolism appears in the tables of critical masses.

$m_{c}$ - critical mass of core

$m_{\max }$ - maximum mass used, in same units as $m_{c}$

$M_{\max }$ - maximum central source neutron multiplication attained

$\mathrm{h}_{c}$ - critical height of cylindric core

d - diameter of core

L $x$ H $\times$ W - length times height times width of parallelepiped

p - density

w/o - weight percent

v/o - volume percent

$\mathrm{U}(93)$ - enriched uranium containing $93 \mathrm{w} / \mathrm{o} \mathrm{U}^{235}$

$U(N)$ - uranium with natural isotopic composition 


\section{TABLE IA1}

HIGHLY ENRICHED U METAL, UNREFLECTED

Corrected empirically for influence of supports and small ( $0.4 ")$ source cavity unless noted otherwise

\begin{tabular}{|c|c|c|c|c|c|c|c|c|}
\hline erence & shape & components & material & $\begin{array}{c}\bar{p}(\text { total } \mathrm{U}) \\
\left(\mathrm{g} / \mathrm{cm}^{3}\right) \\
\end{array}$ & $\mathrm{h}_{\mathrm{c}} / \mathrm{d}$ & $\begin{array}{c}\mathrm{m}_{\mathrm{c}} \\
\left(\mathrm{kg} \mathrm{\textrm {u } ^ { 2 3 5 }}\right)\end{array}$ & $\begin{array}{r}m_{\max } \\
\left(\mathrm{kg} \mathrm{\textrm {U } ^ { 2 3 5 }}\right) \\
\end{array}$ & $\underline{m}_{\text {max }}$ \\
\hline (1) (55) & sphere $^{a}$ & thick sections & $v(93.71)$ & 18.74 & - & $49.12 \pm 0.15$ & critica & \\
\hline (2) (55) & sphere & thick shells & $v(93.86)$ & 28.81 & - & $48.75 \pm 0.15$ & & 142 \\
\hline (3) & pseudosphere & $\sim 0.4 "$ rings $^{b}$ & $v(93.9)$ & $18.5 \pm 0.1$ & - & 50.9 & 49.8 & 150 \\
\hline (4) & psuedosphere & 20.4" rings & $v(93.9)$ & $18.5 \pm 0.1$ & - & 50.6 & 50.0 & 180 \\
\hline (4) & cy.l 4.75" dia & $\sim 0.4^{\prime \prime}$ rings $^{b}$ & $U(93.8)$ & $18.5 \pm 0.1$ & - & $>94$ & 70.8 & 13 \\
\hline (4) & cyl 5.50" dia & $\sim 0.4 "$ rings $^{b}$ & $v(93.8)$ & $18.5 \pm 0.1$ & 1.76 & 66.2 & 61.4 & 96 \\
\hline (4) & cyl 6.37" dia & $20.4^{\prime \prime}$ rings $^{b}$ & $U(94.0)$ & $18.5 \pm 0.1$ & 0.95 & 55.3 & 52.6 & 85 \\
\hline (4) & cyl $7.00^{\prime \prime}$ dia & $\sim 0.4 "$ rings $^{b}$ & $\mathrm{U}(94.0)$ & $18.5 \pm 0.1$ & 0.72 & 55.6 & 54.0 & 76 \\
\hline (4) & cyl 7.50" dia & $\sim 0.4 "$ rings $^{b}$ & $v(94.0)$ & $18.5 \pm 0.1$ & 0.61 & 58.2 & 55.9 & 54 \\
\hline (5) & cyl 15.00" dia & $0.3 \mathrm{~cm}$ plates & U(93.3) & 17.9 & 0.214 & $155.3 \pm 0.6$ & 151 & 67 \\
\hline (5) & cyl 21.00" dia & $0.3 \mathrm{~cm}$ plates & U(93.2) & 17.9 & 0.141 & $281.2 \pm 0.7$ & 270 & 68 \\
\hline
\end{tabular}

a Corrected for slight asphericity

b Uncorrected for 0.06 in $^{3}$ central source cavity; corrected empirically for effect of supports 


\section{TABLE IA2}

$U(93.4)-U(N)$ METAL CYLINDERS, UNREFLECTED

Indicated layers are combinations of $10.5^{\prime \prime}$ diameter, $0.8 \mathrm{~cm}$ thick U(93.4), and $0.6 \mathrm{~cm}$ thick $\mathrm{U}(\mathrm{N})$

Corrected from partial terminating sandwich to fractional sandwich of proper composition

Corrected for reflection effect of support

All systems critical

References (6), (55)

\begin{tabular}{|c|c|c|c|c|c|c|c|}
\hline $\begin{array}{c}\text { average } \\
\text { composition } \\
\end{array}$ & $\begin{array}{r}\text { repeatec } \\
\text { thickne } \\
\text { U(93.4) } \\
\end{array}$ & $\begin{array}{l}\text { yers, } \\
(\mathrm{cm}) \\
U(N) \\
\end{array}$ & $\begin{array}{c}\bar{p}(\operatorname{tota} 1 \mathrm{U}) \\
\left(\mathrm{g} / \mathrm{cm}^{3}\right) \\
\end{array}$ & $\begin{array}{c}\text { diameter } \\
(1 \mathrm{n} .)\end{array}$ & $\begin{array}{c}h_{c} \\
(i n .) \\
\end{array}$ & $\mathrm{h}_{\mathrm{c}} / \mathrm{d}$ & $\begin{array}{c}\mathrm{m}_{\mathrm{c}} \\
\left(\mathrm{kg} \mathrm{\textrm {U } ^ { 2 3 5 }}\right)\end{array}$ \\
\hline$v(53,6)$ & $0.8^{a}$ & 0.6 & 18.7 & 10.50 & 5.10 & 0.581 & $86.8 \pm 1 / 2 \%$ \\
\hline$u(37.7)$ & 0.8 & $1.2^{b}$ & 18.75 & 10.50 & 10.04 & 0.956 & $100.7 \pm 1 / 2 \%$ \\
\hline$U(29.0)$ & 0.8 & $1.8^{b}$ & 18.8 & $11.42 \mathrm{av}^{\mathrm{c}}$ & 13.45 & 1.178 & $123.0 \pm 1 \%$ \\
\hline
\end{tabular}



b starts with $0.6 \mathrm{~cm} U(\mathbb{N})$ at base of stack

c Basic stack of plates extended by blocks of $U(94)$ and $U(N)$ in proper proportion 
TABLE IA3

U(93.3) - U(N) METAL CYLINDERS, 15.00" DIAMETER, UNREFLECTED

Indicated layers, combinations of $0.3 \mathrm{~cm}$ thick $U(93.3)$ and $U(N), 0.6 \mathrm{~cm} U(N)$ or $1.5 \mathrm{~cm} U(N), 5 t a r t$ with $U(N)$ at bottom and end with portion of sandwich at top

Average composition is that of final stack

Corrected for influence of supports of split stack

Communicated by G. A. Jarvis

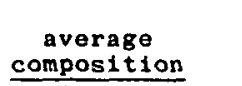

U (93.3)

U $(86.4)$

U $(83.4)$

U $(80.5)$

U (77.7)

U (75.1)

U (70.5)

U $(65.5)$

U (64.4)

U $(56.6)$

$U(57.1)^{c}$

U $(50.5)$

$\mathrm{U}(50.7)^{\mathrm{c}}$

U $(47.0)$

U (47.1)

v $(44.2)$

v $(38.0)$

U $(31.6)$

U (28.9)

U (23.9)

U (21.3)

U $(19.3)$ repeated layers, th 1ckness (cm)
U(93.3) U(N)

0.3

3.6

2.4

1.8

1.5

1.2

0.9

3.6

0.6

2.4

2.1

1.8

1.5

0.6

0.3

1.2

0.9

0.3

0.6

$\left(\begin{array}{l}0.3 \\ 0.3\end{array}\right)$

$(0.3)$

$\left(\begin{array}{l}0.3 \\ 0.3\end{array}\right)$

$\left(\begin{array}{l}0.3 \\ 0.3\end{array}\right)$
0

0.3

0.3

0.3

0.3

0.3

0.3

1.5

0.3

1.5

1.5

1.5

1.5

0.6

0.3

1.5

1.5

0.6

1.5

$\left(\begin{array}{l}1.5 \\ 0.3\end{array}\right)^{b}$

$\left(\begin{array}{l}1.5 \\ 0.6\end{array}\right)^{b}$

$(0.6)$

$\left(\begin{array}{l}1.5 \\ 0.9\end{array}\right)^{b}$ $\bar{p}$ (total U)

$\frac{\begin{array}{c}\left(\mathrm{g} / \mathrm{cm}^{3}\right) \\ 18.06\end{array}}{18}$

18.08

17.95

17.98

17.98

18.19

18.16

18.33

18.21

18.37

18.34

18.35

18.44

18.42

18.25

18.49

18.19

18.51

.18 .32

.18 .65

18,62

18.66 $\mathrm{h}_{\mathrm{c}}$

(in.)

3.18

3.36

3.50

3. 60

3.70

3.77

4.00

4.05

4.34

4.60

4.66

5.25

5.25

5.53

5.61

5.92

7.02

8.23

9.63

11.73

11.15

17.85 $\underline{h_{c} / d}$

0.212

0.224

0.233

0.240

0.247

0.252

0.266

0.270

0.289

0.306

0.311

0.350

0.350

0.369

0.374

0.394

0.468

0.548

0.642

0.782

0.943

1.190 $\frac{\left(k \dot{g} u^{235}\right)}{155.2^{a}}$

152.1

151.6

150.8

149.8

149.1

148.2

140.8

147.6

138.5

141.2

140.9

142.2

138.8

139.8

140.1

142.9

139.2

147.5

151.6

162.6

185.8

a Corrections not as detalled as those for next-to-last item in Table IAl

b The $1.5 \mathrm{~cm} U(N)$ plate was at base of stack; it alternates with the thinner U(N) in successive sandwiches

c Extra U(93.3) plates at top of stack 


\section{TABLE IA4}

U(93.3) - U(N) METAL CILINDERS, 21" DIAMETER, UNREFLECTED

Repeated layers of interleaved $U(93.3)$ and $U(N)$

Referencas (55), (57)

\begin{tabular}{|c|c|c|c|c|c|}
\hline $\begin{array}{c}\text { average } \\
\text { composition }\end{array}$ & $\begin{array}{l}\text { repeated layers, } \\
\text { thickness (in.) }\end{array}$ & $\begin{array}{l}\operatorname{mass} / 1 \text { ayer } \\
(\mathrm{kg} U)\end{array}$ & $\begin{array}{c}\bar{\rho}(\operatorname{tota1} U) \\
\left(\mathrm{g} / \mathrm{cm}^{3}\right)\end{array}$ & $\begin{array}{r}\mathrm{m}_{\mathrm{C}} \\
\left(\mathrm{kg} \mathrm{U}^{235}\right)\end{array}$ & $\begin{array}{l}m_{\max } \\
(\mathrm{kg} \mathrm{U} 235) \\
\end{array}$ \\
\hline $\mathrm{U}(16.01)$ & $\begin{array}{ll}0.591 & U(N) \\
0.118 & U(93.3)\end{array}$ & 76.1 & 18.68 & $232 \pm 2$ & 224 \\
\hline $\mathrm{U}(14.11)$ & $\begin{array}{l}0.709 \mathrm{U}(\mathrm{N}) \\
0.118 \mathrm{U}(93.3)\end{array}$ & 87.0 & 18.41 & $258 \pm 2$ & 252 \\
\hline $\mathrm{U}(12.32)$ & $\begin{array}{l}0.591 U(N) \\
0.118 U(93.3) \\
0.236 U(N)\end{array}$ & 100.4 & 18.64 & $312 \pm 2$ & 307 \\
\hline $\mathrm{U}(10.90)$ & $\begin{array}{ll}0.709 & U(N) \\
0.118 & U(93.3) \\
1.182 & U(N) \\
0.118 & U(93.3)\end{array}$ & 224.8 & 18.63 & $540 \pm 13$ & 412 \\
\hline
\end{tabular}


TABLE IBI

EVRICHED U METAL SPHERE OR PSEUDOSPHERE, U(N) REFLECTOR

\begin{tabular}{|c|c|c|c|c|c|c|c|c|c|}
\hline \multirow[b]{2}{*}{ reference } & \multicolumn{4}{|c|}{ core } & & \multirow{2}{*}{$\left(\mathrm{kg} \mathrm{v^{235 } )}\right.$} & \multirow[b]{2}{*}{$M_{\max }$} \\
\hline & shape & components & material & $\begin{array}{l}\bar{D}(\operatorname{total} U) \\
\left(\mathrm{g} / \mathrm{cm}^{3}\right) \\
\end{array}$ & shape & $\begin{array}{l}\text { reflector } \\
\text { thickness } \\
\text { (in.) } \\
\end{array}$ & $\bar{p}\left(\mathrm{~g} / \mathrm{cm}^{3}\right)$ & & \\
\hline$(7)(55)$ & sphere & hemispheres & $u(93.24)$ & 18.62 & sphere & 7.09 & 19.0 & $16.63 \pm 0.04$ & critical \\
\hline$(2)(55)$ & sphere & nesting shells & $v(93.9)$ & 18.69 & sphere & 3.93 & 19.00 & $18.61 \pm 0.09$ & 167 \\
\hline (2) & sphere & nesting shells & $\mathrm{U}(93.9)$ & 18.75 & sphere & 3.52 & 19.0 & $19.2 \pm 0.2$ & 53 \\
\hline (2) (55) & sphere & nesting shells & $v(93.99)$ & 18.67 & sphere & 1.742 & 18.67 & $24.96 \pm 0.12$ & 141 \\
\hline (2) (55) & sphere & nesting shells & $v(93.91)$ & 18.70 & sphere & 0.683 & 19.00 & $34.31 \pm 0.17$ & 156 \\
\hline (4) & pseudosphere & $0.4 "$ rings & $\mathrm{U}(93.8)$ & $18.5^{a}$ & pseudosphere & 1.87 & 18.7 & 24.6 & 160 \\
\hline (4) & pseudosphere & 20.4" rings & $U(93.8)$ & $18.5^{\mathrm{a}}$ & pseudosphere & 0.99 & 18.7 & 32.4 & 34 \\
\hline (8) & pseudasphere & 1/2" min blocks & $U(94)$ & 18.7 & pseudosphere & $11 \mathrm{av}$ & 19.0 & 16.2 & critical \\
\hline (9) & pseudosphere & 1/2" min blocks & $v(94.13)$ & 18.7 & pseudosphere & 9 av & 19.0 & $16.39 \pm 0.07$ & critical \\
\hline (9) & pseudosphere & $1 / 2 "$ min blocks & $U(80.5)^{b}$ & 18.7 & pseudosphere & $8-3 / 4$ av & 19.0 & 18.3 & critical \\
\hline (9) & pseudosphere & 1/2" min blocks & $\mathrm{U}(67.6)^{\mathrm{b}}$ & 18.75 & pseudosphere & $8-1 / 2$ av & 19.0 & 20.8 & critical \\
\hline (9) & pseudospnere & 1/2" min blocks & $\mathrm{U}(56.6)^{\mathrm{b}}$ & 18.75 & pseudosphere & $8-1 / 2$ av & 19.0 & 21.2 & critical \\
\hline (9) & pseudosphere & 1/2" min blocks & $v(47.3)^{b}$ & 18.8 & pseudosphere & $7-3 / 4$ av & 19.0 & 27.1 & critical \\
\hline (9) & pseudosphere & $1 / 2 "$ min blocks & $\mathrm{U}(94)$ & $16.0^{c}$ & pseudosphere & $8-3 / 4$ av & 19.0 & 19.7 & critical \\
\hline (9) & pseudosphere & $1 / 2 "$ min blocks & $U(94)$ & $15.8^{c}$ & pseudosphere & $8-3 / 4$ av & 19.0 & 20.1 & critical \\
\hline (9) & pseudosphere & 1/2" min blocks & $U(94)$ & $13.1^{\mathrm{c}}$ & pseudosphere & $8-1 / 4$ av & 19.0 & 25.3 & critical \\
\hline (9) & pseudosphere & $1 / 2^{\prime \prime}$ min blocks & $U(94)$ & $9.35^{c}$ & pseudosphere & $7-1 / 4$ av & 19.0 & 37.0 & critical \\
\hline (10) & pseudosphere & $1 / 2 "$ min blocks & $U(78.7)$ & 217.8 & sphere & 19 o.d. & 19.0 & 21.9 & critical \\
\hline
\end{tabular}

ancorrected for 0.06 in. ${ }^{3}$ central source cavity

b Average concentration of mixed $1 / 2$ " cubic units of $U(94)$ and $U(N)$

c Average density with $1 / 2$ " cubic voids distributed throughout core; corrected experimentally for effect of tubular al spacers within voids 
TABLE 182

ENZI GHED U METAL CYLINDER, PSEUDOCYLINDEF, OR PARALLEJEPIPED, U(N) RETLECTOR (LAST ITEM DEPLETED U)

\begin{tabular}{|c|c|c|c|c|c|c|c|c|c|c|}
\hline \multirow[b]{2}{*}{ reference } & \multicolumn{4}{|c|}{ core } & \multicolumn{3}{|c|}{ reflector } & \multirow[b]{2}{*}{ 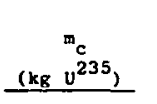 } & \multirow[b]{2}{*}{$\begin{array}{r}\begin{array}{r}m_{\max } \\
\left(k_{8}\right. \\
\left.\mathrm{v}^{235}\right)\end{array} \\
\end{array}$} & \\
\hline & shape & dimenstons $(: n)$. & material & $\begin{array}{l}\overline{0}(\text { tctal } \mathrm{U}) \\
\left(\mathrm{g} / \mathrm{cm}^{3}\right)\end{array}$ & shape & $\begin{array}{c}\text { av thickness } \\
(\text { in. })\end{array}$ & $\begin{array}{r}\overline{0} \\
\left(\mathrm{~g} / \mathrm{cm}^{3}\right) \\
\end{array}$ & & & $H_{\max }^{M_{\max }}$ \\
\hline (8) & pseudocylinder ${ }^{a}$ & $4.00 \times-4.5$ av dia & $U(94)$ & 18.7 & pseudosphere & $-9 \mathrm{av}$ & $19 . \mathrm{C}$ & 16.9 & critical & \\
\hline 18) & parallelepiped & $4.00 \times 4.00 \times 23.5$ & $v(94)$ & 18.7 & pseudosphere & 29 av & 19.6 & 16.9 & critical & \\
\hline (8) & parallelepiped ${ }^{2}$ & $5.00 \times 5.00 \times 22.5$ & $v(94)$ & 18.7 & pseudosphere & $\sim 8-3 / 4$ av & $19 . \bullet$ & 18.2 & critical & \\
\hline (8) & parallelepiped & $7.50 \times 7.50 \times \sim 1.5$ & $U(94)$ & 13.7 & pseudosphere & $28-1 / 4$ av & $19 . n$ & 25.4 & 24.3 & \\
\hline (8) & parallelepiped ${ }^{\mathrm{a}}$ & $7.50 \times 3.00 \times 2=.0$ & $U(94)$ & 18.7 & pseudosphere & $\sim 8-1 / 2$ av & 19.3 & 19.6 & critical & \\
\hline (8) & parallelepiped $^{a}$ & $6.60 \times 3.50 \times 2=.0$ & $U(94)$ & 18.7 & pseudosphere & $\sim 8-3 / 4$ av & 19.2 & 18.2 & critical & \\
\hline (4) & cylinder ${ }^{b}$ & $3.98 \mathrm{d1a}, \mathrm{h}_{\mathrm{c}} / \mathrm{d}-3.51$ & $v(93.7)$ & 18.5 & cylinder & 1.12 & 18.2 & 49.5 & 243 & 67 \\
\hline (4) & cylinder ${ }^{b}$ & $3.98 \mathrm{dia}, \mathrm{r}_{\mathrm{c}} / \mathrm{d}=2.15$ & $v(93.7)$ & 18.5 & cylinder & 1.87 & 13.7 & 30.4 & 230 & 200 \\
\hline (4) & cylinder ${ }^{b}$ & $4.75 \mathrm{dxa}, n_{c} / \mathrm{d}=1.38$ & $U(93.8)$ & 18.5 & cylinder & 1.12 & 18.7 & 33.0 & -31.5 & 59 \\
\hline (4) & $c y l i n d e r^{b}$ & $4.25 \mathrm{dta}, \mathrm{b}_{\mathrm{c}} / \mathrm{d}-1.03$ & U(93.8) & ¿ 8.5 & cylioder & 2.00 & 187 & 24.6 & $\sim 24$ & 100 \\
\hline (4) & cylinder ${ }^{b}$ & $5.50 \mathrm{dia}, h_{c} / d=0.84$ & $U(93.8)$ & 18.5 & cylinder & 1.12 & 187 & 31.3 & 231 & 96 \\
\hline (4) & cylinder ${ }^{b}$ & $5.50 \mathrm{dia},{ }_{\mathrm{c}} / \mathrm{d}-0.67$ & $U(93.8)$ & 28.5 & cylisder & 8.00 & 38.7 & 25.0 & 224 & 96 \\
\hline (4) & cylinder ${ }^{b}$ & $6.37 \mathrm{dia}, h_{c} / d=0.565$ & $U(94.0)$ & 18.5 & cylinder & $: .12$ & 18.7 & 32.4 & 231.5 & 66 \\
\hline (4) & $c y l$ inder ${ }^{b}$ & $6.37 \mathrm{dia} \cdot \mathrm{h}_{\mathrm{c}} / \mathrm{d}=0.47$ & $U(94.0)$ & 18.5 & cylinder & 2.00 & $=8,7$ & 27.4 & 226 & 43 \\
\hline (4) & cylinder ${ }^{b}$ & $7.00 \mathrm{dia}, h_{c} / d=0.46$ & $U(94,0)$ & 18.5 & cylinder & 1.12 & 18.7 & 35.3 & & 38 \\
\hline (4) & cylinder ${ }^{b}$ & $7.50 \mathrm{dia}, \mathrm{h}_{\mathrm{c}} / \mathrm{d}=0.41$ & $u(94.0)$ & 18.5 & cylinder & 1.12 & $1 \varepsilon .7$ & 38.0 & & 107 \\
\hline (4) & Fseudocylinder ${ }^{a}$ & 3.0 av dia, $h_{c} / d-3.08$ & $v(94)$ & 18.7 & pseudosphere & $\sim$ av & $1 \varepsilon .9$ & 21.3 & 20.7 & \\
\hline (4) & pseudocylinder ${ }^{a}$ & 4.0 ay dia, $h_{c^{\prime}} / d-1.00$ & $U(94)$ & 18.7 & pseudosphere & 29 av & $1 E .9$ & 16.66 & crittcal & \\
\hline (4) & pseudocylinder ${ }^{a}$ & 6.5 av dia, $h_{c} / d=0.31$ & $v(94)$ & 18.7 & pseudosphere & $28-1 / 2$ av & 12.9 & 20.3 & 19.6 & \\
\hline (4) & pseudocylinder ${ }^{a}$ & 8.3 av cia, $h_{c} / d=0.18$ & $v(94)$ & 18.7 & pseudosphere & $-7-3 / 4$ av & 23.9 & 25.7 & 24.9 & \\
\hline (11) & cylinder ${ }^{c}$ & $5.25 \mathrm{di \varepsilon}, h_{c} / c=1.25$ & v(93.3) & 18.75 & cylinder & 0.500 & 13.8 & $40.7 \pm 0.1$ & & 1000 \\
\hline (11) & $=y l$ inder ${ }^{c}$ & $5.25 \mathrm{dia}, \mathrm{h}_{\mathrm{c}} / \mathrm{c}=0.965$ & $u(93.3)$ & 18.75 & cylinder & 1.000 & 13.8 & $31.4 \pm 0.1$ & & 500 \\
\hline (12) & cylinder ${ }^{d}$ & $15.00 \mathrm{dia}, \mathrm{h}_{\mathrm{c}} \cdot \mathrm{d}=0.91$ & $v\{93.4)$ & 17.7 & cylinder & 3.00 & 13.9 & $65.4 \pm 1.0$ & & 52 \\
\hline (12) & cylinder ${ }^{e}$ & $3.24 \mathrm{~d} 1 \mathrm{a}, h_{c} / d=8.6$ & $U(93.2)$ & 18.7 & cylinder & 2.75 & LB.9 & $65.5 \pm 1.0$ & & 52 \\
\hline (13)(5s) & eylinder ${ }^{f}$ & $15.00 \mathrm{d1a} \times 12.75$ & $0(16.10)$ & 18.75 & cylinder & 3.00 & 0.0 & $110.6 \pm 0.6$ & critical & \\
\hline
\end{tabular}

"Core of 1,2" min blocks

b Core of $\sim 0.4 "$ nesting riggs; uncorrected for 0.06 in $^{3}$ central source cavity

c Core of discs 1.20" to $0.075 "$ thick: mc corrected empirically for incidental reflectlon, diaphragm supporting pirt of assembly, avd

d Core of $0.3 \mathrm{~cm}$ discs of $\mathrm{j}(93.4)$, $\mathrm{m}_{\mathrm{e}}$ corrected tor influence of support structure

Core or thick plates; reflector $U$ depleted to $20.3 \% \mathrm{U}^{235}$

' Core of alternating $0.3 \mathrm{~cm}$ discs of $v(93.36)$ aod $2.5 \mathrm{~cm}$ olses of $v(\mathrm{M})$ 
TABLE IB3

MISCELLANEOUS ENRICHED U METAL, U RBFIEETOR ${ }^{a}$

reference

(14)

(14)

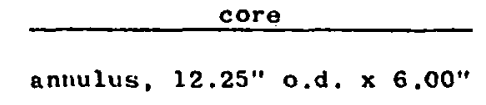

1.d. $x$ 3.01" high, stack of

$1 / 2$ " and 1/4" thick rings

U(93.4), $\bar{D}(\mathrm{U})=18.7 \mathrm{~g} / \mathrm{cm}^{3}$

annulus, 12.25 " o.d. $\times 6.00^{\prime \prime}$

1.d. $x$ 2.03" high, stack of

$2 / 2$ " and $1 / 4$ " thick rings

v(93.4), $\bar{p}(U)-18.7 \mathrm{~g} / \mathrm{cm}^{3}$

pseudocylinder, 13.74" av

d1am $\times 12.00^{\prime \prime}$ av compo-

sition: ${ }^{C} 18.1 \mathrm{v} / 0 \mathrm{U}(93.6)$,

$\overline{0}=3.38 \mathrm{~g} / \mathrm{cm}^{3} ; 13.6 \mathrm{v} / 0$

$U(N), \overline{0}=2.58 \mathrm{~g} / \mathrm{cm}^{3}: 12.8$

$v / 0 \mathrm{Fe}, \overline{\mathrm{p}}=0.92 \mathrm{~g} / \mathrm{cm}^{3}$;

52.3 v/O Al, $\overline{0}-1.40 \mathrm{~g} / \mathrm{cm}^{3}$;

4.2 v/o void

cylinder, 21.00" diam $\times 22.00 "$

long, av composition $v(20.15)$,

$\bar{p}(U)=18.78 \mathrm{~g} / \mathrm{cm}^{3}$ (homogeneous

metal on axis surrounded by

Intcrleaved 0.118 " thick

$U(93.3)$ and $U(N)$ to average

same $U^{235}$ enrichment)

\section{refloctor}

$1.00^{\prime \prime}$ th1ck, U(N), $\bar{D}=19.0$

$\mathrm{g} / \mathrm{cm}^{3}$, completely envelops core

3.00" thick, $U(N), \bar{D}=19.0$

$\mathrm{g} / \mathrm{cm}^{3}$, completely envelops core

$\left(m_{\max }-51.6\right)$

pseudocylinder 5.0" av thickness,

$\bar{D}-18.9 \mathrm{~g} / \mathrm{cm}^{3}$
$52.2 \pm 0.3$

93.0

$\left(u_{\max }-225\right)$

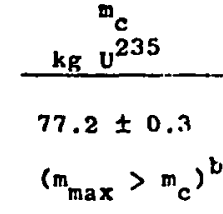

.

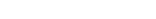

$U(0.30), 6.00 "$ thlck

on periphery, 8.00"

thick on ends

\footnotetext{
Onlisted, is a nonuniform assombly of mixed plates and rings of $U(93.4)$ and U(N) that enclose a noar-central cylindrical cavity. 15.0" diam $x$ 11.8"; outsldo dimonsions of the assembly aro 21.0" diam x 2l" high (S. J. Bilestrini, G. A. Jarvis, J. D. Orndoff, Decembor 1961). Average composition bounding cavity is U(27), 1-1/2" thick U(N) rings form top and bottom of cylinder At critical, the total mass is n.l.100 kg U(N) and $339 \mathrm{~kg} \mathrm{U}(93.1)$. Uncorrected for $1 / 4$ " thick oteel plate supporting portion above cavity.

${ }^{b}$ Corroctod for small gap botwenn assombly halvos.

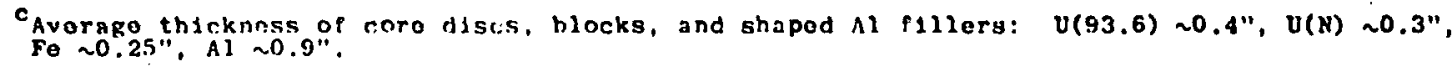


TABLE ICI

HIGHLY EERICHED U METAL, RERLECTOR OF T., W. WC, MO OR $\mathrm{MO}_{2} \mathrm{C}$

\begin{tabular}{|c|c|c|c|c|c|c|c|c|c|c|c|}
\hline \multirow[b]{2}{*}{ ref } & \multicolumn{4}{|c|}{ core } & \multicolumn{4}{|c|}{ reflector } & \multirow[b]{2}{*}{ 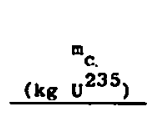 } & \multirow[b]{2}{*}{$\begin{array}{r}\begin{array}{r}m_{\max } \\
\left(\mathrm{kg} \mathrm{U}^{235}\right)\end{array} \\
\end{array}$} & \multirow[b]{2}{*}{$u_{\max }$} \\
\hline & shapz & $\begin{array}{c}\text { dimensions } \\
(\text { in.) }\end{array}$ & ma:erial & $\begin{array}{l}\bar{D}(\operatorname{total} U) \\
\left(\mathrm{g} / \mathrm{cm}^{3}\right) \\
\end{array}$ & material & shape & $\begin{array}{c}\text { thickness } \\
\text { (1n.) }\end{array}$ & $\begin{array}{l}\bar{p}(\text { total } 1) \\
\left(\mathrm{g} / \mathrm{cm}^{3}\right) \\
\end{array}$ & & & \\
\hline (11) & spherc & $\begin{array}{l}\text { (nesting } \\
\text { shells) }\end{array}$ & $U(93.9)$ & 18.6 & Tn & sphere & 1.81 & 11.48 & $34.7 \pm 0.2$ & 34.2 & 162 \\
\hline $\begin{array}{l}\text { (16) } \\
\text { (57) }\end{array}$ & cylinder & $\begin{array}{l}5.967 \mathrm{dia} \\
h_{c} / d-0.59\end{array}$ & $v(93,16)$ & 18.75 & Th & $\begin{array}{l}\text { (21.0" equi- } \\
\text { lateral cyl) }\end{array}$ & & 11.38 & $28.0 \pm 0.3$ & 26.9 & \\
\hline (11) & sphere & $\begin{array}{l}\text { (nesting } \\
\text { shells) }\end{array}$ & $v(93.9)$ & 18.75 & W-alloy & sphere & 2.00 & 17.35 & $24.1 \pm 0.2$ & & 159 \\
\hline (11) & sphere & $\begin{array}{l}\text { (nesting } \\
\text { shells) }\end{array}$ & $U(93.9)$ & 18.75 & $M-a 110 y^{a}$ & sphere & 4.00 & 17.35 & 19.4 & 18.3 & 44 \\
\hline (11) & cylinder & $\begin{array}{l}5.25 \text { dia, } \\
h_{c} / d-1.25 b\end{array}$ & $v(93.3)$ & 18.75 & $H$-alloy ${ }^{c}$ & cylinder & 0.500 & 17.3 & $40.6 \pm 0.8$ & . & 1250 \\
\hline (11) & cylinder & $\begin{array}{l}5.25 \mathrm{dia} \\
\mathrm{h}_{\mathrm{c}} / \mathrm{d}=0.97^{\mathrm{b}}\end{array}$ & U(93.3) & 18.75 & $17-a 110 y^{c}$ & cylinder & 1.000 & 17.3 & $31.75 \pm 0.1$ & & 128 \\
\hline (17) & crlinde- & $4.25 \mathrm{dia}$ & U(93.5) & 18.7 & H-alloy & cylinder & $\begin{array}{l}2.00 \text { but } \\
\text { one end } 3.00\end{array}$ & 17.3 & 27.36 & critical & \\
\hline (8) & sphere & $\begin{array}{l}\text { (shc1ls) } \\
0.83 \text { i.d. }\end{array}$ & U(93.9) & 18.45 & wC & pseudosphere & $2.9 \mathrm{av}$ & 214.7 & 18.7 & 15.1 & 13 \\
\hline$i 81$ & sphere & $\begin{array}{l}(\text { she } 11 \mathrm{~s}) \\
0.83 \mathrm{i} . \mathrm{d} .\end{array}$ & U:93.9) & 18.45 & $w c$ & pseudosphere & $4.5 \mathrm{av}$ & 214.7 & 16.6 & 15.1 & 29 \\
\hline (8) & sphere & $\begin{array}{l}\text { (she11s) } \\
0.83 \text { i.d. }\end{array}$ & $U(93.9)$ & 18.45 & wC & pseudosphere & $6.5 \mathrm{av}$ & 214.7 & 16.3 & 15.1 & 36 \\
\hline (10) & pseudosphere & $\begin{array}{l}(1 / 2 * \text { min } \\
\text { blocks) }\end{array}$ & $U(78.5)$ & 17.8 & $\mathrm{WC}$ & (14" cube) & & $14 .:$ & $20.8^{\mathrm{e}}$ & eritical & \\
\hline (17) & cylinder & $4.25 \mathrm{dia}$ & $u(93.5)$ & 18.7 & wC & cylinder & 2.00 & $214 .:$ & 24.4 & 23.6 & 80 \\
\hline (11) & cylinder & $\begin{array}{l}5.25 \text { dia } \\
h_{c} / d-1.29^{b}\end{array}$ & $\tau(93.3)^{\circ}$ & 18.75 & $\begin{array}{l}\text { Mo } \\
(99.8 w / 0)\end{array}$ & cylinder & 0.500 & 10.33 & 41.7 & & 210 \\
\hline (11) & cylinder & $\begin{array}{l}5.25 \text { dia. } \\
h_{c} d d=1.0 .1 b\end{array}$ & U(93.3: & 18.75 & $\begin{array}{l}\text { Mo } \\
(99.8 \text { w/o) }\end{array}$ & cylinder & 1.000 & $10 . .53$ & 32.9 & & 141 \\
\hline (11:) & eylinder & $\begin{array}{l}5.25 \mathrm{dia} \\
\mathrm{h}_{\mathrm{c}} / \mathrm{d}=1.23^{\mathrm{b}}\end{array}$ & T(93.3: & 18.75 & $\mathrm{HO}_{2} \mathrm{C}^{\mathrm{f}}$ & cylinder & 0.500 & 9.57 & 39.9 & & 270 \\
\hline$(11)$ & cylinder & $\begin{array}{l}5.25 \mathrm{dia} \\
\mathrm{h}_{\mathrm{c}} / \mathrm{d}=0.95^{\mathrm{b}}\end{array}$ & U(93.3) & 18.75 & $\mathrm{HO}_{2} \mathrm{C}^{f}$ & cylinder & 1.000 & 9.57 & 30.9 & & 110 \\
\hline
\end{tabular}

- Composition 90 w/a 11,7 w/o NI, 3 w/o Cu

b Core of dises 1.22 " to $0.075 "$ thick: mc corrected empirically for incidental reflection, diaphragm supporting part of assemply, and
0.05 in. $3^{3}$ central cavity



d Composition 92 w/a $\mathrm{k}, 5.5$ w/o $\mathrm{Ni}, 2.5 \mathrm{w} / \mathrm{C}$ ou

For cylindurs in this reflector, $m_{c} / m_{c}$ (sphere) $=0.98,0.96,0.93$ when $h_{c} / d-0.92,0.63,1.60$. respectively

f Composition 95 to $96 \% / 0 \mathrm{NO}_{2}=4$ to 5 w/O Ni 
TABLE IC2

HIGHLY ENRICHED U METAL; REFLECTOR OF Zn, Cu, Ni, Co, OR Fe

\begin{tabular}{|c|c|c|c|c|c|c|c|c|c|c|c|}
\hline \multirow[b]{2}{*}{ ref } & \multicolumn{4}{|c|}{ core } & \multicolumn{4}{|c|}{ reflector } & \multirow[b]{2}{*}{$\begin{array}{r}\mathrm{m}_{\mathrm{c}} \\
\left(\mathrm{kg} \mathrm{U}^{235}\right) \\
\end{array}$} & \multirow[b]{2}{*}{$\begin{array}{r}\begin{array}{r}m_{\max } \\
\left(k_{B} \mathrm{U}^{235}\right)\end{array} \\
\end{array}$} & \multirow[b]{2}{*}{$u_{\max }$} \\
\hline & _ shape & $\begin{array}{c}\text { dimensions } \\
\text { (in.) }\end{array}$ & material & $\begin{array}{l}\bar{D}(\text { total } \mathrm{U}) \\
\left(\mathrm{g} / \mathrm{cm}^{3}\right) \\
\end{array}$ & material & shape & $\begin{array}{c}\text { thickness } \\
\text { (in.) }\end{array}$ & $\begin{array}{l}\bar{D}(\text { total }) \\
\left(\mathrm{g} / \mathrm{cm}^{3}\right)\end{array}$ & & & \\
\hline (11) & spherc & $\begin{array}{l}\text { (nesting } \\
\text { shells) }\end{array}$ & $u(93.9)$ & 18.7 & 2n & sphere & 2.00 & 7.04 & 30.0 & 28.5 & 52 \\
\hline (11) & sphere & $\begin{array}{l}\text { (nesting } \\
\text { shells) }\end{array}$ & $u(93.9)$ & 18.5 & $\mathrm{zn}$ & sphere & 4.075 & 7.04 & $25.4 \pm 0.3$ & 23.9 & 46 \\
\hline $\begin{array}{l}(11) \\
\text { (35) }\end{array}$ & sphere & $\begin{array}{l}\text { (nesting } \\
\text { shells) }\end{array}$ & $U(93,8)$ & 28.38 & $\mathrm{cu}$ & sphere & 1.980 & 8.88 & $26.4 \pm 0.2$ & & 118 \\
\hline $\begin{array}{l}\text { (11) } \\
\text { (5s) }\end{array}$ & sphere & $\begin{array}{l}\text { (nesting } \\
\text { shells) }\end{array}$ & $U(94.0)$ & 18.43 & $c u$ & sphere & 4.158 & 8.88 & $20.8 \pm 0.2$ & & 141 \\
\hline (11) & cylinder & $\begin{array}{l}5.25 \mathrm{dia} \\
\mathrm{h}_{\mathrm{c}} / \mathrm{d}-\mathrm{l} .29^{\mathrm{a}}\end{array}$ & $v(93.3)$ & 18.75 & $a^{b}$ & cylinder & 0.500 & 8.87 & $42.16 \pm 0.1$ & & 330 \\
\hline (11) & cylinder & $\begin{array}{l}5.25 \text { dia. } \\
h_{c}^{\prime} d-1.03^{a}\end{array}$ & $U(93.3)$ & 18.75 & $\omega^{b}$ & cylinder & 1.000 & 8.87 & $33.44 \pm 0.1$ & & 190 \\
\hline (8) & pseudospherc & $\begin{array}{l}(1 / 2 " \text { min } \\
\text { blocks) }\end{array}$ & $\mathrm{v}(94.0)$ & 18.7 & "A"-Ni & pseudosphere & $8-3 / 4$ av & 8.88 & 19.9 & critical & \\
\hline $\begin{array}{l}\text { (11) } \\
\text { (5s) }\end{array}$ & sphere & $\begin{array}{l}\text { (nesting } \\
\text { shells) }\end{array}$ & $v(93.8)$ & 28.38 & $-A^{\prime \prime}-H 1$ & sphere & 1.945 & 8.90 & $27.5 \pm 0.4$ & 25.9 & 42 \\
\hline (11) & cylinder & $\begin{array}{l}5.25 \text { dia. } \\
h_{c} / d=1.29^{a}\end{array}$ & $0(93.3)$ & 18.75 & Ni (elect) & cylinder & 0.500 & 8.79 & 42.0 & & 170 \\
\hline (11) & cylinder & $\begin{array}{l}5.25 \mathrm{dia}, \\
\mathrm{h}^{\prime} \mathrm{d}^{\prime}-1.04^{\mathrm{a}}\end{array}$ & $v(93.3)$ & 18.75 & N1 (elect) & cylinder & 1.000 & 8.79 & 34.0 & & 190 \\
\hline (11) & cylinder & $\begin{array}{l}5.25 \text { dia. } \\
h_{c} / d=1.27^{a}\end{array}$ & $U(93.3)$ & 18.75 & Co (reag) & cylinder & 0.500 & 8.72 & 41.5 & & 102 \\
\hline (11) & cylinder & $\begin{array}{l}5.25 \text { dia } \\
h_{c} / d=1.02^{a}\end{array}$ & U(93.3) & 18.75 & Co (reag) & cylinder & 1.000 & 8.72 & 33.3 & & 117 \\
\hline (11) & sphere & $\begin{array}{l}\text { (nesting } \\
\text { shells) }\end{array}$ & $u(93.9)$ & 18.6 & Fe (cast) & sphere & 2.00 & 7.16 & $29.7 \pm 0.3$ & 28.5 & 59 \\
\hline (11) & sphere & $\begin{array}{l}\text { (nesting } \\
\text { shells) }\end{array}$ & U(93.9) & 18.4 & Fe (cast) & sphere & 4.00 & 7.16 & $26.0 \pm 0.2$ & & 143 \\
\hline (18) & sphere & $\begin{array}{l}\text { (thick } \\
\text { shelis) }\end{array}$ & $U(93.9)$ & 18.52 & steel & (60" cube) & & -7.7 & 23.4 & & 64 \\
\hline (11) & cylindẹ & $\begin{array}{l}5.25 \text { dia. } \\
h_{c} / d=1.42^{a}\end{array}$ & $U(93.3)$ & 18.75 & $\mathrm{Fe}^{\mathrm{C}}$ & cylinder & 0.500 & 7.78 & $46.3 \pm 0.2$ & & 105 \\
\hline (11) & cylinder & $\begin{array}{l}5.25 \text { dia, } \\
h_{c} / d=1.18^{a}\end{array}$ & $v(93.3)$ & 18.75 & $\mathrm{Fe}^{\mathrm{c}}$ & cylinder & 1.000 & 7.78 & $38.38 \pm 0.1$ & & 340 \\
\hline (17) & cylinder & $4.25 \mathrm{dia}$ & U(93.5) & 18.7 & $\mathbf{P e}^{\mathbf{c}}$ & cylinder & 4.00 & 7.78 & 33.6 & 26.9 & 13 \\
\hline
\end{tabular}

a Core of giscs $1.20 "$ to $0.075 "$ thick; mc corrected empirically for locidental reflection, diaphragen supporting part of assembly, and
0.05 in. 3 central cavity

Cast or, 12 to 1 wo inpuriey

c Steel, SAE 1020 
TABLE ICZ

HIGHLY ENRICHED $"$ METAL, REPLECTOR OF $\mathrm{T} i, \wedge 1, \wedge_{2} \mathrm{O}_{3}, \mathrm{Mg}_{\mathrm{g}}, \mathrm{Be}, \mathrm{OR}$ BeO

\begin{tabular}{|c|c|c|c|c|c|c|c|c|c|c|}
\hline \multirow[b]{2}{*}{ ref } & \multicolumn{4}{|c|}{ core } & \multicolumn{4}{|c|}{ reflectur } & \multirow[b]{2}{*}{$\left(\mathrm{kg}^{\mathrm{m}} \mathrm{v}^{235}\right)$} & \multirow[b]{2}{*}{$M_{\max }$} \\
\hline & shape & $\begin{array}{c}\text { dimensions } \\
(\text { in. })\end{array}$ & matertal & $\begin{array}{l}\overline{0}(\text { total U) } \\
\left(\mathrm{g} / \mathrm{cm}^{3}\right) \\
\end{array}$ & materlal & Shapo & $\begin{array}{c}\text { thickness } \\
\text { (in.) }\end{array}$ & $\begin{array}{l}\bar{F}(\operatorname{totan}) \\
\left(\mathrm{g} / \mathrm{cm}^{3}\right)\end{array}$ & & \\
\hline (11) & cylinder & $\begin{array}{l}5.25 \text { dia } \\
h_{c} / d-1.61^{B}\end{array}$ & $U(93.3)$ & 18.75 & $\mathrm{Ti}^{\mathrm{b}}$ & cylinder & 0.500 & 4.50 & $52.4 \pm 0.6$ & 16 \\
\hline (11) & cylinder & $\begin{array}{l}5.25 \mathrm{diu}, 38^{\mathrm{a}} \\
\mathrm{h}_{\mathrm{c}} / \mathrm{d}-1.3 \mathrm{~s}^{2}\end{array}$ & $v(03,3)$ & 18.75 & $T^{b}$ & cylinder & 1.000 & 4.50 & $45.0 \pm 0.1$ & 125 \\
\hline (19) & sphere & $\begin{array}{l}\text { (nesting } \\
\text { shells) }\end{array}$ & $v(93.18)$ & 28.40 & A1 (2014) & sphere & $\begin{array}{l}2.610 \\
\pm 0.03\end{array}$ & 2.82 & $34.71 \pm 0.1$ & 170 \\
\hline (11) & cylinder & $\begin{array}{l}5.25 \text { dia, } \\
h_{c} / d \cdot 1.59^{a}\end{array}$ & $v(93.3)$ & 18.75 & 11 (2s) & cylinder & 0.500 & 2.70 & $52.0 \pm 0.6$ & 17 \\
\hline (11) & oylintar & 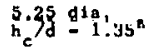 & $v(93.3)$ & 18.75 & $A 1$ (2S) & cylinder & 1.000 & 2.70 & $44.1 \pm 0.1$ & 200 \\
\hline (11) & cylindor & $\begin{array}{l}5.25 d 1 \mathrm{a}, \\
\mathrm{h}_{\mathrm{c}} / \mathrm{d}-1.40^{\mathrm{a}}\end{array}$ & $v(93.3)$ & 18.75 & $\mathrm{Al}_{2} \mathrm{O}_{3}$ & cylinder & 0.500 & 2.76 & 45.5 &. .100 \\
\hline (11) & cylinder & $\begin{array}{l}5.25 d 1 a \\
h_{c} / d-1.14^{a}\end{array}$ & $\mathrm{v}(93.3)$ & 18.75 & ${ }^{A} 1_{2} O_{3}$ & cylinder & 1.000 & 2.76 & 37.2 & -150 \\
\hline (11) & cylinder & $\begin{array}{l}5.25 d 1 \mathrm{a} \\
\mathrm{h}_{c} / d=1.66^{\mathrm{a}}\end{array}$ & $v(93.3)$ & 18.75 & $\mathrm{Hg}_{\mathrm{g}}(\mathrm{FS}-1)$ & cylinder & 0.500 & 1.77 & $54.2 \pm 0.7$ & 13 \\
\hline (11) & cylinder & $\begin{array}{l}5.25 \mathrm{dia} \\
\mathrm{h}_{\mathrm{c}} / \mathrm{d}-1.46^{\mathrm{a}}\end{array}$ & $v(93,3)$ & 18.75 & $M g(F S-1)$ & cylinder & 1.000 & 1.77 & $47.7 \pm 0.3$ & 34 \\
\hline (11) & sphere & $\begin{array}{l}\text { (nesting } \\
\text { shells) }\end{array}$ & $v(93.9)$ & 18.5 & Be & sphere & 1.85 & 1.84 & $22.2 \pm 0.2$ & 100 \\
\hline (11) & sphere & $\begin{array}{l}\text { (nesting } \\
\text { shells) }\end{array}$ & $v(93.9)$ & 18.75 & $\mathrm{Bc}$ & sphere & 1.89 & 1.84 & 21.6 & 24 \\
\hline (11) & sphere & $\begin{array}{l}\text { (nesting } \\
\text { shells) }\end{array}$ & $v(93.6)$ & 18.6 & $\mathrm{Be}$ & sphere & 4.84 & 1.84 & $13.1 \pm 0.2^{c}$ & 143 \\
\hline (11) & cylinder & $\begin{array}{l}5.25 \text { dia } \\
h_{c} / d-1.19^{a}\end{array}$ & $v(93.3)$ & 18.75 & Be (ouv) & cylinder & 0.500 & 1.84 & $38.89 \pm 0.1$ & 480 \\
\hline (II) & cylinder & $\begin{array}{l}5.25 d i a, \\
{ }_{c} / d=0.90^{a}\end{array}$ & $v(93,3)$ & 28.75 & De (QHV) & cylinder & 1.000 & 1.84 & $39.38 \pm 0.1$ & 210 \\
\hline (12) & cylinder & $\begin{array}{l}15.00 d 1 a \\
h_{c} / d=0.131\end{array}$ & $v(93.4)$ & 17.7 & Be & cylinder & 1.00 & 1.80 & $93.9 \pm 0.9$ & 25 \\
\hline (12) & cylinder & $\begin{array}{l}15.00 \mathrm{dia} \\
h_{c} / d-0.090\end{array}$ & $v(93,4)$ & 17.7 & Be & cylinder & 2.00 & 1.80 & $64.9 \pm 1.0$ & 23 \\
\hline (12) & cylinder & $\begin{array}{l}15.00 d 10 \\
h_{c} / d=0.068\end{array}$ & $v(93.4)$ & 17.7 & Be & cylinder & 3.00 & 1.80 & $49.0 \pm 1.0$ & 35 \\
\hline (12) & eylinder & $\begin{array}{l}15.00 d i a \\
h_{c} / d-0.053\end{array}$ & $u(93.4)$ & 17.7 & Be & cylinder & 4.00 & 1.80 & $37.8 \pm 0.5$ & 13 \\
\hline (12) & cylinder & $\begin{array}{l}15.00 \mathrm{dia} \\
h_{c} / d-0.042\end{array}$ & $U(93.4)$ & 17.7 & Be & cylinder & 5.00 & 1.80 & $30.4 \pm 0.5$ & 15 \\
\hline (11) & pgaudresphere & $\begin{array}{l}\left(1 / 2 " 2^{m} g^{n}\right. \\
\text { blocks) }\end{array}$ & $U(94)$ & 18.7 & Beo & psuudosphere & $2.35 \mathrm{av}$ & 2.69 & 19.7 & 85 \\
\hline (11) & pseudosphere &  & $U(94)$ & 18.7 & Beo & pseudosphere & $3: 520$ & 2.69 & 16.5 & 105 \\
\hline (10) & pseudusphere & $\begin{array}{l}(1 / 2 " \min \\
\text { blocks) }\end{array}$ & $u(82.7)$ & 17.8 & $\mathrm{BeO}$ & (24" cube) & & 0.2 .69 & 10.3 & oritical \\
\hline
\end{tabular}

are of discs $1,20 "$ to $0.075 "$ thick; mc corrected empirically for incidertal reflection, diaphragm supporting part of assembly.

b Composition $96.5 \% / \mathrm{Ti}, 2.5 \% \mathrm{Cr}, 1 \% / \mathrm{Fe}$

c with $0.010^{\circ}$ cd botweon core and reflector, $m_{c}-14.0 \times g U^{235}$ (1)

duncorrected for 0.06 in. $^{3}$ central source cavity 
TABLE IC4a

HIGHLY ENRICHED U METAL, COMPLETE GRAPHITE REFLECTOR

Graphite is grade Cs-312 except as noted

\begin{tabular}{|c|c|c|c|c|c|c|c|c|c|}
\hline \multirow[b]{2}{*}{ re? } & \multicolumn{4}{|c|}{ core } & \multicolumn{3}{|c|}{ reflector } & \multirow[b]{2}{*}{$\left(\mathrm{kg} \mathrm{u}^{\mathrm{m}} \mathrm{c}\right)$} & \multirow[b]{2}{*}{${ }_{\max }^{M}$} \\
\hline & shape & $\begin{array}{c}\text { dimensions } \\
(\text { in.) }\end{array}$ & material & $\begin{array}{l}\bar{D}(\text { total } \mathrm{U}) \\
\left(\mathrm{g} / \mathrm{cm}^{3}\right) \\
\end{array}$ & shape & $\begin{array}{l}\text { thickness } \\
\text { (in.) }\end{array}$ & $\frac{\bar{n}}{\left(\mathrm{~s} / \mathrm{cm}^{3}\right)}$ & & \\
\hline$(20)$ & sphere & $\begin{array}{l}\text { (nesting } \\
\text { she11s)a }\end{array}$ & $U(93.9)$ & 18.7 & sphere & 2.00 & 1.67 & $29.6 \pm 0.3$ & 58 \\
\hline$(20):$ & sphere. & $\begin{array}{l}\text { (nesting } \\
\text { shellg)a }\end{array}$ & $U(93.9)$ & 18.7 & sphere & 4.00 & 1.67 & $\begin{array}{c}24.3 \pm 0.2 \\
.\end{array}$ & 150 \\
\hline (20) & sphere & $\begin{array}{l}\text { (nesting } \\
\text { shells)a }\end{array}$ & $U(93.9)$ & 18.45 & sphere & 6.00 & 1.67 & $21.5 \pm 0.2$ & 150 \\
\hline$(20)$ & sphero & $\begin{array}{l}\text { (nesting } \\
\text { shells) }\end{array}$ & $v(93.9)$ & 18.75 & sphere & 8.00 & 1.67 & $19.5 \pm 0.3$ & 42 \\
\hline (20) & sphere & $\begin{array}{l}\text { (nesting } \\
\text { sholls) }\end{array}$ & $U(93.9)$ & 18.5 & pseudosphere & 17 av & 1.66 & 17.0 & 48 \\
\hline $\begin{array}{c}(20) \\
\ldots\end{array}$ & cylindar & $\begin{array}{l}3.25 d 1 a,{ }^{c} \\
h_{c} / d=2.95\end{array}$ & $v(93.7)$ & 18.5 & pseudosphereb & 17 av & 1.66 & 22.5 & 17 \\
\hline$(20)$ & pseudocylinder & $\begin{array}{l}3.62 \text { av diad } \\
h_{c} / d=1.85 d\end{array}$ & $U(94)$ & 18.7 & pseudosphere ${ }^{b}$ & 17. av & 1.68 & 20.1 & 40 \\
\hline$(20)$ & cylinder & $\begin{array}{l}3.98 \text { dia, } \\
h_{c} / d=1.30^{c}\end{array}$ & $v(93.7)$ & 18.5 & pseudosphere ${ }^{b}$ & 17 av & 1.68 & 18.3 & 109 \\
\hline$(20)$ & cylinder & $\begin{array}{l}4.75 \mathrm{~d} 1 \mathrm{a} \\
\mathrm{h}_{\mathrm{c}} / \mathrm{d}=0.815^{c}\end{array}$ & $U(93.7)$ & 18.5 & pseudosphere & 17 av & 1.66 & 17.5 & 82 \\
\hline$(20)$ & cylinder & $\begin{array}{l}5.50 \mathrm{~d} 1 \mathrm{a}, \\
\mathrm{h}_{\mathrm{c}} / \mathrm{d}=0.495^{c}\end{array}$ & $v(93.8)$ & 18.5 & pscudosphere ${ }^{b}$ & 17 av & 1.66 & 18.5 & 78 \\
\hline$(20)$ & cylinder & $\begin{array}{l}6.375 d 1 a_{1} c \\
h_{c} / d=0.345^{c}\end{array}$ & $v(94.0)$ & 18.5 & preudosphere ${ }^{\circ}$ & 27 av & 1.66 & 20.0 & 107 \\
\hline$(20)$ & cylinder & $\begin{array}{l}7.50 d 1 a_{1} \\
h_{c} / d=0.235^{c}\end{array}$ & $v(94.0)$ & 18.5 & psoudosphere & $17 \mathrm{av}$ & 1.66 & 22.7 & 150 \\
\hline (20) & pseudocylinder & $h_{c}=1.50,1.177^{d}$ & $U(94.0)$ & 18.7 & pyeudosphere & 17 av & 1.66 & 24.6 & 90 \\
\hline (20) & pseudocylinder & $h_{c}=1.00 .1081^{d}$ & $v(94.0)$ & 18.7 & pseudosphere & 17 av & 1.66 & 34.8 & 200 \\
\hline (11) & cylinder & $\begin{array}{l}5.25 d i a, \\
h_{c} / d-1.42^{e}\end{array}$ & $U(93.3)$ & 18.75 & cylinder & 0.500 & 1.67 & $46.35 \pm 0.2$ & 51 \\
\hline$(11)$ & cylinder & $\begin{array}{l}5.25 d a_{1}{ }^{e} \\
h_{c} / d=1.16^{e}\end{array}$ & $U(93,3)$ & 18.75 & cylindor & 1.000 & 1.67 & $37.71 \pm 0.1$ & $>500$ \\
\hline (12) & cylinder & $\begin{array}{l}3.24 d i a, \\
h_{c} / d=6.78\end{array}$ & $U(93.2)$ & 18.7 & cylinder & 4.85 & 1.60 & $\$ 1.7 \pm 0.9$ & 233 \\
\hline (12) & cylinder & $\begin{array}{l}3.24 d i a, \\
h_{c} / d-4.97\end{array}$ & $U(93.2)$ & 18.7 & cylinder & 5.75 & 1.60 & $37.9 \pm 0.7$ & 1350 \\
\hline (12) & cylinder & $\begin{array}{l}3.24 d 1 a, \\
h_{c} / d=4.41\end{array}$ & $u(93.2)$ & 18.7 & cylinder & 6.25 & 1.80 & $33.6 \pm 0.7$ & 460 \\
\hline (12) & cylinder & ${ }_{h}^{13.00 d i n}=0.073 t$ & $U(93,4)$ & 17.7 & cyllndor & 7.00 & 1.60 & $62.1 \pm 1.0$ & 20 \\
\hline (21) & cylinder & ${ }_{h_{c} / d}^{10.50 d i a}=0.192^{R}$ & $v(93.4)$ & 18.7 & cylinder & 2.00 & 1.68 & so. 0 & 18 \\
\hline$(14)$ & annulus & $\begin{array}{l}12.25 \text { o.d. } x \\
6.00 \text { i.d. } x \\
2.86\end{array}$ & $v(93.4)$ & 18.7 & $\begin{array}{l}\text { (onvelops } \\
\text { core) }\end{array}$ & 2.00 & 21.67 & $73.3 \pm 0.3$ & (max \\
\hline
\end{tabular}

ancorrected for $0.05 \mathrm{in} .^{3}$ central source cavity

b Pile-grade graphite surrounds 25 " thick CS -312

c Interlocking rings; uncorrected for $0.06 \mathrm{in}^{3}$ central source cavity

d Formed of 1/2" inin blocks

e Core of discs 1.20" to 0.075" thick: m corrected umpirically for Incidental roflection, diaphragm supporting part of assembly, and 0.05 in. 3 central cavity

f Core of $0.3 \mathrm{~cm}$ thick plates; empirical correction for dinphragm supporting part of assumbly

G Core of 0.315 " thick plates: empirical correction for diaphragm supporting part of assumbly 
TABLE ICAt.

hiGHLY ENRICHED U METAL, PARTIA.L GRAPHITE REFLECTOR

Reflector same diameter as core where on ends only

Corrected empirically for diaphragm supporting part of assembly, for incidental reflection, and for small source cavity (no correction required for last iten)

\begin{tabular}{|c|c|c|c|c|c|c|c|c|c|c|}
\hline & \multirow[b]{2}{*}{ ref } & \multicolumn{4}{|c|}{ core } & \multicolumn{3}{|c|}{ reflector } & \multirow[b]{2}{*}{$\begin{array}{c}m_{c} \\
\left(\mathrm{~kg} \mathrm{\textrm {v } ^ { 2 3 5 }}\right) \\
\end{array}$} & \multirow[b]{2}{*}{$M_{\max }$} \\
\hline & & shape & $\begin{array}{c}\text { dimensions } \\
(\text { in.) }\end{array}$ & material & $\begin{array}{l}\overline{0}(\text { total U) } \\
\left(\mathrm{g} / \mathrm{cm}^{3}\right)\end{array}$ & $\begin{array}{c}\text { surfaces } \\
\text { reflected }\end{array}$ & $\begin{array}{c}\text { thickness } \\
\text { (in.) }\end{array}$ & $\begin{array}{c}\overline{\bar{o}} \\
\left(\mathrm{~g} / \mathrm{cm}^{3}\right)\end{array}$ & & \\
\hline & (5) & cylinder & $15.00 \mathrm{dia}$ & $u(93.3)$ & $17.9 \pm 0.2$ & top plane & 1.00 & 1.79 & $135.5 \pm 0.5$ & 123 \\
\hline & (5) & cylinder & $15.00 \mathrm{dia}$ & $U(93.3)$ & $17.9 \pm 0.2$ & top plane & 2.00 & 1.79 & $125.4 \pm 0.5$ & 46 \\
\hline & (5) & cylinder & $21.00 \mathrm{dia}$ & $\mathrm{U}(93.2)$ & $18.2 \pm 0.2$ & top plane & 2.00 & 1.73 & $222.3 \pm 0.6$ & 140 \\
\hline \multirow{12}{*}{$\vec{\sigma}$} & (5) & cylinder & $15.00 \mathrm{dia}$ & $U(93.3)$ & $17.9 \pm 0.2$ & both planes & 6.00 & 1.7 & $75.4 \pm 0.3$ & 37 \\
\hline & (5) & cylinder & $21.00 \mathrm{dia}$ & $v(93.2)$ & $18.2 \pm 0.2$ & both planes & 6.00 & 1.7 & $103.5 \pm 0.3$ & 46 \\
\hline & (5) & cylinder & $15.00 \mathrm{dia}$ & $v(93.3)$ & $17.9 \pm 0.2$ & top plane & 7.00 & 1.71 & $113.9 \pm 0.4$ & 43 \\
\hline & (5) & cylinder & $21.00 \mathrm{dia}$ & $\mathrm{U}(33.2)$ & $18.2 \pm 0.2$ & top plane & 7.00 & 1.76 & $190.2 \pm 0.6$ & 95 \\
\hline & (5) & cylinder & $21.00 \mathrm{dia}$ & $\mathrm{U}(93.2)$ & $18.2 \pm 0.2$ & top plane & 8.00 & 1.75 & $188.4 \pm 0.6$ & 101 \\
\hline & (5) & cylinder & $15.00 \mathrm{dia}$ & $\mathrm{U}(93.3)$ & $17.9 \pm 0.2$ & tof plane & 12.00 & 1.70 & $113.4 \pm 0.4$ & 52 \\
\hline & (5) & cylinder & $21.00 \mathrm{dia}$ & $U(93.2)$ & $18.2 \pm 0.2$ & top plane & 12.00 & 1.76 & $185.7 \pm 0.6$ & 67 \\
\hline & (5) & cylinder & $15.00 \mathrm{dia}$ & $\mathrm{U}(93.3)$ & $17.9 \pm 0.2$ & top plane & 24.00 & 1.71 & $113.3 \pm 0.4$ & 54 \\
\hline & (5) & cylinder & $21.00 \mathrm{dia}$ & $\mathrm{U}(93.2)$ & $18.2 \pm 0.2$ & top plane & 14.00 & 1.76 & $185.3 \pm 0.6$ & 76 \\
\hline & (21) & cylinder & $\begin{array}{l}10.50 \mathrm{dia} \\
h_{c} / d=0.226\end{array}$ & $U(93.4)$ & 18.7 & both planes & 2.00 & 1.68 & 58.7 & 20 \\
\hline & (22) & annulus & $\begin{array}{l}21.000 . d . x \\
15.001 . d . \times \\
3.44\end{array}$ & $U(93.16)$ & 17.9 & $\begin{array}{l}\text { (across both } \\
\text { planes) }\end{array}$ & 6.00 & 1.7 & 164.6 & 330 \\
\hline & (23) & annulus & $\begin{array}{l}6.14 \text { o.d. } x \\
3.85 \text { i.d. } x \\
6.36\end{array}$ & $\mathrm{U}(93.15)$ & 18.7 & $\begin{array}{l}\text { top, bottom, } \\
\text { wali (none } \\
\text { inside) }\end{array}$ & $\begin{array}{l}9.5 \\
8.9\end{array}$ & 1.67 & $32.7 \pm 0.3$ & 28 \\
\hline
\end{tabular}


TABLE IC5

HIGHLY ENRICHED U METAL, REFLECTOR OF $\mathrm{D}_{2} \mathrm{O}(99.8 \%)(24)$

\begin{tabular}{|c|c|c|c|c|c|c|c|}
\hline \multicolumn{4}{|c|}{ core } & \multirow[b]{2}{*}{$\begin{array}{l}\text { reflector } \\
\text { thickness } \\
\text { (in.) }\end{array}$} & \multirow{2}{*}{ (sphere) } & \multirow[b]{2}{*}{$\begin{array}{c}\mathrm{m}_{\mathrm{c}} \\
\left(\mathrm{kg} \mathrm{U}^{235}\right) \\
\end{array}$} & \multirow[b]{2}{*}{$M_{\max }$} \\
\hline shape & $\begin{array}{c}\text { dimensions } \\
\text { (in.) } \\
\end{array}$ & material & $\begin{array}{c}\bar{\rho}(\operatorname{total} \mathrm{U}) \\
\left(\mathrm{g} / \mathrm{cm}^{3}\right) \\
\end{array}$ & & & & \\
\hline sphere & $\begin{array}{l}\text { (nesting } \\
\text { shells) }\end{array}$ & $\mathrm{U}(93.9)^{\mathrm{a}}$ & 18.5 & 3.28 & $0.04^{\prime \prime} \mathrm{ss}$ & 23.3 & 36 \\
\hline sphere & $\begin{array}{l}\text { (nesting } \\
\text { shells) }\end{array}$ & $v(93.9)^{a}$ & 18.5 & 4.59 & $0.10^{\prime \prime} \mathrm{Al}$ & 20.5 & 22 \\
\hline sphere & $\begin{array}{l}\text { (nesting } \\
\text { shells) }\end{array}$ & $U(93.9)^{a}$ & 18.5 & 5.50 & $0.04^{\prime \prime} \mathrm{ss}$ & 19.0 & 55 \\
\hline sphere & $\begin{array}{l}\text { (nesting } \\
\text { shells) }\end{array}$ & $\mathrm{U}(93.9)^{a}$ & 18.5 & 6.84 & $0.04 "$ ss & 17.1 & 40 \\
\hline sphere & $\begin{array}{l}\text { (nesting } \\
\text { shells) }\end{array}$ & $\mathrm{U}(93.7)^{\mathrm{a}}$ & 18.5 & 15.3 & $0.2^{\prime \prime} \mathrm{ss}$ & 13.4 & $>400$ \\
\hline $\begin{array}{l}\text { sphere } \\
\text { surrounded } \\
\text { by } 0.010^{\prime \prime C d}\end{array}$ & $\begin{array}{l}\text { (nesting } \\
\text { shells) }\end{array}$ & $\mathrm{U}(93.9)^{\mathrm{a}}$ & 18.5 & 6.7 & $0.04^{\prime \prime}$ ss & 20.9 & $m_{\max }=18.0$ \\
\hline $\begin{array}{l}\text { sphere } \\
\text { surrounded } \\
\text { by } 0.010^{\prime \prime C d}\end{array}$ & $\begin{array}{l}\text { (nesting } \\
\text { shells) }\end{array}$ & $U(93.9)^{a}$ & 18.5 & 15.1 & $0.2^{\prime \prime} \mathrm{ss}$ & 20.2 & $m_{\max }=18.0$ \\
\hline $\begin{array}{l}\text { hollow sphere, } \\
\text { filled with } \mathrm{D}_{2} \mathrm{O}\end{array}$ & $3.60 \mathrm{i} . \mathrm{d}$ & $\mathrm{U}(93.9)$ & 18.5 & 14.9 & $0.2^{\prime \prime}$ ss & 16.4 & 16 \\
\hline $\begin{array}{l}\text { hollow sphere, } \\
\text { filled with } \mathrm{D}_{2} \mathrm{O}\end{array}$ & $4.08 \mathrm{i} . \mathrm{d}$ & $U(93.7)$ & 18.5 & 14.7 & $0.2^{\prime \prime} \mathrm{ss}$ & 17.2 & 32 \\
\hline $\begin{array}{l}\text { hollow sphere, } \\
\text { filled with } \mathrm{D}_{2} \mathrm{O}\end{array}$ & 4.97 i.d. & $U(93.7)$ & 18.5 & 14.4 & $0.2 "$ ss & 18.3 & 18 \\
\hline
\end{tabular}

a Empirical correction for snall central source cavity 


\section{TABLE IC68}

HIGHLY ENRICHED U METAL, COMPLETE REFLFCTOR OF $\mathrm{H}_{2} \mathrm{O}$ ON POLYETHYLENE see also first it tem of Table IIFs

\begin{tabular}{|c|c|c|c|c|c|c|c|c|c|c|}
\hline \multirow[b]{2}{*}{ ref } & \multicolumn{4}{|c|}{ core } & \multicolumn{4}{|c|}{ reflector } & \multirow[b]{2}{*}{$\left(k_{B} u^{235}\right)$} & \multirow[b]{2}{*}{${ }_{\max }^{n_{\max }}$} \\
\hline & shape & $\begin{array}{l}\text { dimensions } \\
\text { (in.) }\end{array}$ & material & $\begin{array}{l}\overline{0}(\operatorname{total} \mathrm{U}) \\
\left(\mathrm{g} / \mathrm{cm}^{3}\right) \\
\end{array}$ & materlal & shape & $\begin{array}{l}\text { thickness } \\
(1 \mathrm{n} .)\end{array}$ & $\begin{array}{c}\bar{D} \\
\left(\mathrm{~g} / \mathrm{cm}^{3}\right) \\
\end{array}$ & & \\
\hline (25) & sphere & $\begin{array}{l}\text { (sholls) } \\
0.831 . d .\end{array}$ & $\mathrm{U}(93.9)$ & 18.5 & $\mathrm{H}_{2} \mathrm{O}$ & cylinder & $>12$ & 1.00 & 23.4 & 49 \\
\hline$(25)$ & $\begin{array}{l}\text { sohere } \\
\text { surrounded } \\
\text { by } 0.010^{*} \mathrm{~cd}\end{array}$ & $\begin{array}{l}\text { (sholls) } \\
\text { U.BS } 1.4 .\end{array}$ & $v(93.9)$ & 18.4 & $\mathrm{H}_{2} \mathrm{O}$ & cylinder & $>12$ & 1.00 & 32.0 & 32 \\
\hline (20) & sphere & $\begin{array}{l}\text { (nesting } \\
\text { shells) }\end{array}$ & $U(93.9)$ & 18.5 & $\mathrm{H}_{2} \mathrm{O}$ & cylinder & $>12$ & 1.00 & 23.2 & 154 \\
\hline (24) & sphere & $\begin{array}{l}\text { (nesting } \\
\text { shells)a }\end{array}$ & U(93.9) & 18.5 & $\mathrm{H}_{2} \mathrm{O}$ & sphere & 3.25 & 1.00 & 23.5 & 35 \\
\hline (24); & 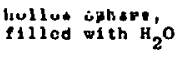 & $3, \operatorname{fn} \backslash A$ & $v(93.9)$ & 18.5 & $\mathrm{H}_{7} \mathrm{O}$ & sphere & 14.6 & 1.00 & 25.1 & 40 \\
\hline (24) & $\begin{array}{l}\text { hollow sphere, } \\
\text { f11led with } \mathrm{H}_{2} \mathrm{O}\end{array}$ & $4.081 . \mathrm{d}$ & $v(93.9)$ & 18.5 & $\mathrm{H}_{2} \mathrm{O}$ & sphere & 14.4 & 1.00 & 26.3 & 80 \\
\hline (24) & $\begin{array}{l}\text { hollow sphere, } \\
\text { filled with } \mathrm{H}_{2}^{\prime} \mathrm{O}\end{array}$ & $4.68 \mathrm{l} . \mathrm{d}$ & $U(93.8)$ & 18.5 & $\mathrm{H}_{2} \mathrm{O}$ & sphere & 14.3 & 1.00 & 27.7 & 19 \\
\hline (20) & cylinder & $\begin{array}{l}3.98 d 1 a, \\
h_{c} / d-1.90^{a}\end{array}$ & $U(93.7)$ & 18.5 & $\mathrm{H}_{2} \mathrm{O}$ & cylunder & $>12$ & 1.00 & 26.7 & 200 \\
\hline (20) & cylinder & $\begin{array}{l}4.75 \text { dia, } \\
h_{c} / d=0.98^{a}\end{array}$ & $U(93.8)$ & 18.5 & $\mathrm{H}_{2} \mathrm{O}$ & cylinder & $>12$ & 1.00 & 23.7 & 101 \\
\hline$(20)$ & cylinder & $\begin{array}{l}5.50 \mathrm{~d} 1 \mathrm{a}, \\
h_{c} / \mathrm{d}=0.66^{a}\end{array}$ & $U(93,8)$ & 18.5 & $\mathrm{H}_{2} \mathrm{O}$ & cylinder & $>12$ & 1.00 & 24.4 & 200 \\
\hline (20) & cylinder & $\begin{array}{l}6.375 \mathrm{dia} \\
h_{c} / d-0.46^{2}\end{array}$ & $U(94,0)$ & 18.5 & $\mathrm{H}_{2} \mathrm{O}$ & cylinder & $>12$ & 1.00 & 25.9 & 130 \\
\hline (20) & cylinder & $\begin{array}{l}7.00 \mathrm{dda} \\
h_{c^{\prime}} \mathrm{d}-0.365^{\mathrm{a}}\end{array}$ & $U(94.0)$ & 18.5 & $\mathrm{H}_{2} \mathrm{O}$ & cylinder & $>12$ & 1.00 & 27.7 & 108 \\
\hline (20) & cylinder & $\begin{array}{l}7.50 \mathrm{dxa} \\
{ }_{\mathrm{c}} / \mathrm{d}=0.300^{\circ}\end{array}$ & $U(94.0)$ & 18.5 & $\mathrm{H}_{2} \mathrm{O}$ & cylinder & $>12$ & 1.00 & 29.0 & 53 \\
\hline (as) & annulus & $\begin{array}{l}6.14 \text { o.d. } x \\
3.851 . d . x \\
5.76 \mathrm{~b}\end{array}$ & $U(93.15)$ & 18.75 & $\mathrm{H}_{2} \mathrm{O}$ & cylinder & $>12$ & 1.00 & $2 \dot{y} .6 \pm 0.3$ & 25 \\
\hline$(26)$ & $\begin{array}{l}\text { hemisholl, } \\
\text { segmented }\end{array}$ & $\begin{array}{l}12.0 \text { o.d. } \\
10.0 \mathrm{i.d.}\end{array}$ & $U(93.5)$ & 18.75 & $\mathrm{H}_{2} \mathrm{O}$ & cylinder & $>6$ & 1.00 & 36 & 20 \\
\hline (12) & cylindor & $\begin{array}{l}3.24 d i a \\
h_{c} / d-12.2\end{array}$ & $u(93.2)$ & 18.7 & $\mathrm{H}_{2} \mathrm{O}$ & crlinder & $>12$ & 1.00 & $93.2 \pm 5$ & 43 \\
\hline (12) & cylinder & $\begin{array}{l}15.000 d a, a^{c} \\
h_{c} / d-0.082^{c}\end{array}$ & $U(93.4)$ & 17.7 & $\mathrm{H}_{2} \mathrm{O}$ & cylinder & $>12$ & 1.00 & $39.0 \pm 0.5$ & 170 \\
\hline (11) & cylindor & $\begin{array}{l}5.25 \text { dia. } \\
h_{c} / d-1.34^{d}\end{array}$ & $v(93.3)$ & 18.75 & polyethylene & cylinder & 0.500 & 0.921 & 43.7 & 140 \\
\hline (11) & cylinder & $\begin{array}{l}5.25 \mathrm{~d} i \mathrm{a}, 0 \mathrm{~d}^{\mathrm{d}} \\
\mathrm{h}^{\prime} / \mathrm{d}-1.00^{-1}\end{array}$ & $U(93.3)$ & 18.75 & polyethylene & cylinder & 1.000 & 0.921 & 32.7 & 140 \\
\hline (12) & cyllndor & $\begin{array}{l}3.24 d 1 a . \\
h_{c} / d=8.0\end{array}$ & $v(93,2)$ & 18.7 & pulyechigl ane & oylendor & $\Delta$ nn & 0.92 & $61.3 \pm 0.9$ & 161 \\
\hline$(12)$ & cylindur & $\begin{array}{l}18.00 \\
h_{r} / d\end{array}$ & $11(93.4)$ & 17.7 & polyethylene & eylinder & 2.00 & 0.92 & $68.4 \pm 0.9$ & 79 \\
\hline (14) & onnulus & $\begin{array}{l}12.250 . d . \times \\
6.001, d . \times \\
2.20\end{array}$ & $U(93.4)$ & 18.7 & polyethylene & $\begin{array}{l}\text { (envelops } \\
\text { core) }\end{array}$ & 3.00 & 0.02 & $36.6 \pm 0.3$ & $>\lim _{c}$ \\
\hline
\end{tabular}

Q Uncorrocted for $0.05 \mathrm{in}^{3}$ central source cavity

bater jilis animulua

c Core of $0.3 \mathrm{~cm}$ plates; empirical correction for small source cevity and diaphrage supporting part of assembly (not used 11 th

a Empirical corroction for small contral caolty and auppont afforta

- corrected for small a ap in tinal confleuration 
TABLE IC6b

HIGHLY ENRICHED U METAL, COMPLETE REFLECTOR OF PARAFFIN

Parafin reflector cylindrical, $>8^{\prime \prime}$ thick, $\bar{\rho}=0.89 \mathrm{~g} / \mathrm{cm}^{3}$

\begin{tabular}{|c|c|c|c|c|c|c|}
\hline \multirow[b]{2}{*}{ ref } & \multicolumn{4}{|c|}{ core } & \multirow[b]{2}{*}{$\begin{array}{l}m_{c} \\
\left.v^{235}\right)\end{array}$} & \multirow[b]{2}{*}{$M_{\max }$} \\
\hline & shape & $\begin{array}{c}\text { dimensions } \\
(\text { in.) }\end{array}$ & material & $\begin{array}{c}\bar{p}(\operatorname{tota1} U) \\
\left(\mathrm{g} / \mathrm{cm}^{3}\right)\end{array}$ & & \\
\hline$(20)$ & sphere & $\begin{array}{l}\text { (nesting } \\
\text { shells)a }\end{array}$ & $U(93.9)$ & 18.5 & 22.2 . & 62 \\
\hline (25) & sphere & $\begin{array}{l}\text { (shells) } \\
0.831 . \mathrm{d} .\end{array}$ & $U(93.9)$ & 18.5 & 22.8 & 69 \\
\hline$(20)$ & cylinder & $\begin{array}{l}3.25 d i a, b \\
h_{c} / d=4.4\end{array}$ & $U(93.7)$ & 18.5 & 35 & 11 \\
\hline (20) & cylinder & $\begin{array}{l}3.98 d i a, \\
h_{c} / d=1.80^{b}\end{array}$ & $U(93.7)$ & 18.5 & 25.0 & 77 \\
\hline$(20)$ & cylinder & $\begin{array}{l}4.75 \mathrm{~d} 1 \mathrm{a}, \\
\mathrm{h}_{\mathrm{c}} / \mathrm{d}=0.915^{\mathrm{b}}\end{array}$ & $U(93.8)$ & 18.5 & 22.2 & 108 \\
\hline$(20)$ & cylinder & $\begin{array}{l}5.50 \mathrm{~d} i \mathrm{a}, \\
\mathrm{h}_{\mathrm{c}} / \mathrm{d}=0.605^{\mathrm{b}}\end{array}$ & $U(93.8)$ & 18.5 & 22.4 & 123 \\
\hline$(20)$ & cylinder & $\begin{array}{l}6.375 \mathrm{dia},{ }^{b} \\
h_{c} / d=0.45^{b}\end{array}$ & $U(94.0)$ & 18.5 & 24.5 & 200 \\
\hline (20) & cylinder & $\begin{array}{l}7.50 \text { dia, } \\
h_{c} / d=0.280^{b}\end{array}$ & $U(94.0)$ & 18.5 & 26.9 & 86 \\
\hline
\end{tabular}

a Uncorrected for 0.05 in. $^{3}$ central sourcc cavity

b Interlocking rings $0.4^{\prime \prime}$ thick, uncorrected for 0.05 in. ${ }^{3}$ central source cavity 
TABLE ICGC

highly ENRiched U Metal, Partial Reflictor of $\mathrm{H}_{2} \mathrm{O}$, pOlyethylene, lucite, OR Paraffin (5) Reflector same diameter as core

Core of $0.3 \mathrm{~cm}$ plates and rings; $m_{c}$ corrected empirically for diaphragm supporting part of assembly, for incidental reflection, and for small central source cavit

\begin{tabular}{|c|c|c|c|c|c|c|c|c|}
\hline \multirow[b]{2}{*}{$\begin{array}{r}\text { cylinder } \\
\text { dia (in.) }\end{array}$} & \multicolumn{2}{|l|}{ core } & \multicolumn{4}{|c|}{ reflector } & \multirow[b]{2}{*}{$\mathrm{kg} \mathrm{u^{235 } )}$} & \multirow[b]{2}{*}{$M_{\max }$} \\
\hline & material. & $\begin{array}{c}\overline{\bar{p}(\operatorname{tota} 1 \mathrm{u})} \\
\left(\mathrm{g} / \mathrm{cm}^{3}\right) \\
\end{array}$ & material & $\begin{array}{r}\text { surfaces } \\
\text { reflected } \\
\end{array}$ & $\begin{array}{c}\text { thickness } \\
\text { (in.) }\end{array}$ & $\begin{array}{c}\bar{\mu} \\
\left(\mathrm{g} / \mathrm{cm}^{3}\right) \\
\end{array}$ & & \\
\hline 15.00 & $\mathrm{U}(93.3)$ & $17.9 \pm 0.2$ & $\mathrm{H}_{2} \mathrm{O}^{\mathrm{a}}$ & top plane & 6.00 & 1.00 & $109.6 \pm 0.4$ & 250 \\
\hline 21.00 & $v(93.2)$ & $18.2 \pm 0.2$ & $\mathrm{H}_{2} \mathrm{O}^{\mathfrak{a}}$ & top plane & 6.00 & 1.00 & $188.5 \pm 0.7$ & 99 \\
\hline 15.00 & $v(93.3)$ & $17.9 \pm 0 . \dot{2}$ & polyethylene & top plane & 1.00 & 0.008 & $208.0 \pm 0.5$ & 167 \\
\hline 21.00 & $v(93.2)$ & $18.2 \pm 0.2$ & polyethylene & top plane & 1.00 & 0.925 & $228.4 \pm 0.6$ & 125 \\
\hline 15.00 & $v(93.3)$ & $17.9 \pm 0.2$ & polyethylene & top plane & 2.00 & 0.925 & $113.6 \pm 0.4$ & 57 \\
\hline 21.00 & $U(93.2)$ & $18.2 \pm 0.2$ & polyethylene & top plane & 2.00 & 0.925 & $198.5 \pm 0.6$ & 35 \\
\hline 15.00 & $v(93.3)$ & $17.9 \pm 0.2$ & polyethylene & both planes & 2.00 & 0.925 & $73.1 \pm 0.3$ & 73 \\
\hline 21.00 & $U(93.2)$ & $18.2 \pm 0.2$ & polyethylene & both planes & 2.00 & 0.925 & $127.4 \pm 0.3$ & 90 \\
\hline 15.00 & $v(93.3)$ & $17.9 \pm 0.2$ & polyethylene & top plane & 3.00 & 0.925 & $109.3 \pm 0.4$ & 77 \\
\hline 21.00 & $v(93.2)$ & $18.2 \pm 0.2$ & polyethylene & top plane & 3.00 & 0.925 & $190.3 \pm 0.6$ & 280 \\
\hline 15.00 & $v(93.3)$ & $17.9 \pm 0.2$ & polyethylene & top plane & 4.00 & 0.925 & $108.5 \pm 0.4$ & 75 \\
\hline 21.00 & $v(93.2)$ & $18.2 \pm 0.2$ & polyothylene & top plane & 4.00 & 0.925 & $188.5 \pm 0.6$ & 160 \\
\hline 15.00 & $\mathrm{v}(93.3)$ & $17.9 \pm 0.2$ & polyethylene & top plane & 6.00 & 0.925 & $308.7 \pm 0.4^{b}$ & 99 \\
\hline 21,00 & $\mathrm{v}(93.2)$ & $18.2 \pm 0.2$ & polyethylene & top plane & 6.00 & 0.925 & $187.9 \pm 0.6^{c}$ & 195 \\
\hline 15.00 & $\mathrm{v}(93.3)$ & $17.9 \pm 0.2$ & polyethylene & top plane & 8.00 & 0.925 & $108.5 \pm 0.4$ & 110 \\
\hline 21.00 & $v(\theta 3.2)$ & $18.2 \pm 0.2$ & polyethylene & top plane & 8.00 & 0.925 & $187.8 \pm 0.6$ & 245 \\
\hline 15.00 & $v(93,3)$ & $17.9 \pm 0.2$ & polyethylene & top plane & 10.00 & 0.925 & $108.5 \pm 0.4$ & 120 \\
\hline 21.00 & $U(93.2)$ & $18.2 \pm 0.2$ & polyethylene & top plane & 10.00 & 0.925 & $187.6 \pm 0.6$ & 102 \\
\hline 15.00 & $u(93.3)$ & $17.9 \pm 0.2$ & lucite & top plane & 6.00 & 1.28 & $106.4 \pm 0.4$ & 52 \\
\hline 21.00 & $\mathrm{u}(93.2)$ & $18.2 \pm 0.2$ & lucite & top plane & 6.00 & 1.18 & $182.1 \pm 0.6$ & 74 \\
\hline 15.00 & $v(93.3)$ & $17.9 \pm 0.2$ & paraftin & lop plane & 6.00 & 0.87 & $109.2 \pm 0.4$ & 130 \\
\hline 21.00 & $U(93,2)$ & $18.2 \pm 0.2$ & parafin & top plane & 6.00 & 0.87 & $188.5 \pm 0.6$ & 49 \\
\hline
\end{tabular}

\footnotetext{
"Empirical correction for effect of 1/16" Al tank containing water, via influence of the tank containing lucite

b Critical mass $129.0 \pm 0.5\left(M_{\max }=170\right)$ when $0.015 "$ cd between core and reflector

c Erjtical mass $22.4 \pm 0.6\left(M_{\text {max }}=38\right)$ when 0.015 " Cd between core and reflector
} 
TABLE IC7

HIGHLY ENRICHED $U$, MIXED REFLECTOR ${ }^{\text {a }}$

\begin{tabular}{|c|c|c|c|c|c|c|c|c|c|c|}
\hline \multirow[b]{2}{*}{ ref } & \multicolumn{4}{|c|}{ core } & \multicolumn{4}{|c|}{ reflector } & \multirow[b]{2}{*}{$\begin{array}{r}{ }^{m_{c}} \\
\left(k g u^{235}\right) \\
\end{array}$} & \multirow[b]{2}{*}{$\underline{\operatorname{miax}} \cdot$} \\
\hline & shape & $\begin{array}{l}\text { dimensions } \\
\text { (in.) }\end{array}$ & mater1al & $\begin{array}{l}\bar{\rho}(\text { total } v) \\
\left(\mathrm{g} / \mathrm{cm}^{3}\right) \\
\end{array}$ & shape & $\begin{array}{c}\text { thickness } \\
(1 \mathrm{In} .)\end{array}$ & material & $\begin{array}{c}\bar{D} \\
\left(\mathrm{~g} / \mathrm{cm}^{3}\right) \\
\end{array}$ & & \\
\hline (27) & sphere & $\begin{array}{l}\text { (nesting } \\
\text { she11s) }\end{array}$ & $U(93,9)$ & 18.4 & sphere & 1.88 & $\begin{array}{llll}40 & \nabla / 0 & \mathrm{Cu} \\
32 & w / 0 & \mathrm{NI} \\
28 & \nabla / 0 \mathrm{Zn}\end{array}$ & 8.55 & 26.7 & 89 \\
\hline (27) & sphere & $\begin{array}{l}\text { (nest1ng } \\
\text { shells) }\end{array}$ & $u(93.9)$ & 18.75 & sphere & 2.02 & $\begin{array}{llll}40 & \nabla / 0 & \mathrm{Cu} \\
32 & \nabla / 0 & \mathrm{Ni} \\
28 & \nabla / 0 & \mathrm{Zn}\end{array}$ & 8.55 & 25.7 & 46 \\
\hline (28) & sphere & $\begin{array}{l}\text { (she11s) } \\
0.83 \text { i.d. }\end{array}$ & $u(93.9)$ & 18.45 & $\begin{array}{l}\text { Inside: sphere } \\
\text { outside: }\end{array}$ & 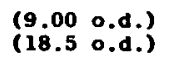 & $\begin{array}{l}v(k) \\
\text { Al. }\end{array}$ & $\begin{array}{r}19.0 \\
2.7\end{array}$ & 20.2 & 22 \\
\hline (29) & sphere & $\begin{array}{l}\text { (shells) } \\
0.831 . d .\end{array}$ & $U(93.9)$ & 18.5 & $\begin{array}{l}\text { Ins1de: } \\
\text { sphere } \\
\text { outside: }\end{array}$ & 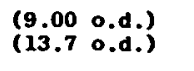 & $\begin{array}{l}\mathrm{v}(\mathrm{H}) \\
\mathrm{A1}\end{array}$ & $\begin{array}{r}19.0 \\
2.7\end{array}$ & 21.8 & 65 \\
\hline (29) & sphere & $\begin{array}{l}\text { (shel1s) } \\
0.83 \text { i.d. }\end{array}$ & $U(93.9)$ & 18.5 & $\begin{array}{l}\text { Inside: } \\
\text { outside: }\end{array}$ & 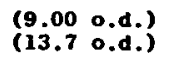 & $\begin{array}{l}U(N) \\
B e\end{array}$ & $\begin{array}{l}19.0 \\
1.84\end{array}$ & 17.7 & 300 \\
\hline (30) & sphere & $\begin{array}{l}\text { (thick } \\
\text { shells) }\end{array}$ & $U(93.2)$ & 18.4 & $\begin{array}{l}\text { Inside: sphere } \\
\text { outside: sphere }\end{array}$ & $\begin{array}{l}0.50 \\
1.30_{5}\end{array}$ & $\begin{array}{l}\mathrm{U}(\mathrm{N}) \\
\mathrm{Be}\end{array}$ & $\begin{array}{l}29.0 \\
1.84\end{array}$ & 23.0 & \\
\hline (11) & sphere & $\begin{array}{l}\text { (nesting } \\
\text { shells) }\end{array}$ & $v(93.5)$ & 28.8 & $\begin{array}{l}\text { Inside: } \text { sphere } \\
\text { outside: } \\
\text { sphere }\end{array}$ & $\begin{array}{l}2.00 \\
2.00\end{array}$ & $\begin{array}{l}\text { T-alloyc } \\
\text { cast iron }\end{array}$ & $\begin{array}{r}17.39 \\
7.16\end{array}$ & $21.0 \pm 0.5$ & 20 \\
\hline (11) & cylinder & $\begin{array}{l}5.25 d i a \\
h_{c} / d-0.99\end{array}$ & $0(93.3)$ & 18.75 & $\begin{array}{l}\text { Inside: cylinder } \\
\text { outside: cylinder }\end{array}$ & $\begin{array}{l}0.500 \\
0.500\end{array}$ & $\begin{array}{l}\mathrm{Be} \\
\mathrm{Pe}\end{array}$ & $\begin{array}{l}1.84 \\
7.78\end{array}$ & 32.4 & 102 \\
\hline (14) & annulus & $\begin{array}{l}12.250 . d . . \\
6.001 . d . . \\
h_{c}=1.98\end{array}$ & $v(93.4)$ & 18.7 & $\begin{array}{l}\text { 1nside: (envelops } \\
\text { outside: core) }\end{array}$ & $\begin{array}{l}1.00 \\
2.00\end{array}$ & $\begin{array}{l}\text { U(N) } \\
\text { polyethylene }\end{array}$ & $\begin{array}{l}19.0 \\
0.92\end{array}$ & 50.9 & $\operatorname{ming}_{c}$ \\
\hline (31) & cylinder & $15.00 \mathrm{dia}$ & U(93.3) & $" 17.7$ & (cyl, top plane only) & $2.0(31 \mathrm{lb})$ & concrete $^{e}$ & 2.3 & $127.9^{f}$ & 96 \\
\hline (31) & cylinder & $15.00 \mathrm{dla}$ & $U(93.3)$ & 17.7 & (cyl, top plane only) & $4.0\left(\begin{array}{ll}58 & 1 b\end{array}\right)$ & concrete $^{\theta}$ & 22.3 & $119.5^{\mathrm{t}}$ & 10 \\
\hline (31) & cylinder & $15.00 \mathrm{~d} 1 \mathrm{a}$ & U(93.3) & 17.7 & (cyl, top plane only) & $6.0(891 b)$ & concrete $^{e}$ & 22.3 & $117.5^{P}$ & 20 \\
\hline (31) & cylinder & $15.00 \mathrm{dIa}$ & $v(93.3)$ & 17.7 & (cyl, top plane only) & 8.0 (116 1b) & concrete $^{e}$ & $\sim 2.3$ & $116.4^{f}$ & 29 \\
\hline (31) & cylinder & $15.00 \mathrm{dla}$ & U\{93.3\} & 17.7 & (cyl, top plane only) & $12.0(1781 b)$ & concrete $^{e}$ & $\sim 2.3$ & $116.1^{7}:$ & 35 \\
\hline (31) & cylinder & $15.00 \mathrm{dla}$ & U(93.3) & 17.7 & (cyl, top plane only) & $28.0(406 \mathrm{lb})$ & concrete $^{\theta}$ & $\sim 2.3$ & $115.8^{r}$ & 40 \\
\hline
\end{tabular}

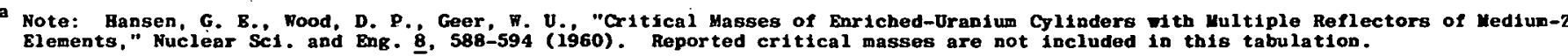

b Uncorrected for $0.05 \mathrm{in}^{3}{ }^{3}$ central source cavity

c Composition $90 \mathrm{~W} / \mathrm{N}, 7 \mathrm{W/0} \mathrm{N1}, 3 \mathrm{~W} / \mathrm{O} \mathrm{C}$

d corrected for small gap in pinal configuration

e Class A concrete: 1548 1b 3/4" rock, 1563 lb sand, 517 lb Portland cement, 40.3 gal water

${ }^{f}$ Unreflected, $m_{c}-152.8\left(w_{\max }-168\right)$; curves of $1 / 1$ us mass paralleled for this series 
TABLE IIAI

U(93.3) METAL CYLINDERS DILUTED $W=T H \mathrm{Fe}, \mathrm{Ni}$, CU, OR $\mathrm{Zn}$, 15" DIAMETER, UNREFLECTED

Thickness of U193.3) plates $0.3 \mathrm{~cm}$

Plate of diluent at base, portion of sandwich at top, unless noted otherwise

Average composition is that of final stack

Correctec for influence of supports of split stack

Communiceted by G. A. Jarvis

\begin{tabular}{|c|c|c|c|c|c|c|c|c|c|}
\hline diluent (A) & $\begin{array}{r}\text { vol } \% \\
\mathrm{U}(93.3) \\
\end{array}$ & $\begin{array}{r}\text { repeate } \\
\text { thickn } \\
0(93.3) \\
\end{array}$ & $\begin{array}{r}\text { ayers, } \\
(\mathrm{cm})\end{array}$ & $\begin{array}{c}\bar{D}(\mathrm{U}) \\
\left(\mathrm{g} / \mathrm{cm}^{3}\right) \\
\end{array}$ & $\begin{array}{c}\bar{p}(\mathrm{~A}) \\
\left(\mathrm{g} / \mathrm{cm} \mathrm{m}^{3}\right) \\
\end{array}$ & $\begin{array}{c}b_{c} \\
\left(i_{0}\right) \text {. }\end{array}$ & $h_{c^{\prime}}$ & $\left(\mathrm{kg} \mathrm{v^{235 } )}\right.$ & $\begin{array}{c}m_{\max } \\
(\mathrm{kg} \\
\left.\mathrm{v}^{235}\right)\end{array}$ \\
\hline $\mathrm{Fe}$ & 72.8 & 2.4 & 0.95 & 13.28 & 2.08 & 4.33 & $0.28 \subseteq$ & 155.3 & $15 I$ \\
\hline $\mathrm{Fe}$ & 62.3 & 1.5 & 0.95 & 11.36 & 2.88 & 5.21 & 0.347 & 159.9 & 157 \\
\hline $\mathrm{Fe}$ & 49.0 & 0.9 & 0.95 & 8.97 & 3.91 & 7.02 & $0.46 \varepsilon$ & 170.0 & 169 \\
\hline $\mathrm{Fe}$ & 49.0 & $0.9^{\mathrm{a}}$ & 0.95 & 8.97 & 3.91 & 7.00 & $0.46=$ & 169.6 & 163 \\
\hline $\mathrm{Fe}$ & 39.1 & 0.6 & 0.95 & 7.18 & 4.68 & 9.84 & 0.656 & 190.8 & 187 \\
\hline $\mathrm{Fe}$ & 38.4 & $0.5^{\mathrm{a}}$ & 0.95 & 7.01 & 4.70 & 9.96 & 0.654 & 188.7 & 182 \\
\hline $\mathrm{Ni}$ & 72.4 & 2.7 & 0.95 & 13.15 & 2.35 & 4.33 & 0.283 & 153.7 & 151 \\
\hline $\mathrm{Ni}$ & 61.8 & 1.5 & 0.95 & 11.33 & 3.29 & 5.14 & 0.343 & 157.5 & 157 \\
\hline $\mathrm{Ni}$ & 48.1 & 0.7 & 0.95 & 8.82 & 4.47 & 6.82 & 0.455 & 162.6 & 157 \\
\hline $\mathrm{Mi}$ & 39.1 & 0.6 & 0.95 & 7.28 & 5,26 & 9.20 & 0.611 & 178.8 & 175 \\
\hline $\mathrm{Ni}$ & 38.3 & $0.6^{\mathrm{a}}$ & 0.95 & 7.07 & 5.34 & 9.28 & 0.617 & 177.3 & 169 \\
\hline cu & 79.4 & 3.6 & 0.95 & 13.96 & 1.775 & 3.85 & 0.250 & 150.2 & 145 \\
\hline $\mathrm{Cu}$ & 72.3 & 2.4 & 0.95 & 12.56 & 2.40 & 4.26 & 0.284 & 151.8 & $i 45$ \\
\hline ou & 66.4 & 1.8 & 0.95 & 12.13 & 2.92 & 4.68 & 0.312 & 153.4 & 151 \\
\hline $2 u$ & 61.2 & 1.5 & 0.95 & $1: .21$ & 3.37 & 5.06 & $0.33=8$ & 153.5 & 151 \\
\hline $\mathrm{Cu}$ & 57.4 & 1.2 & 0.95 & 10.50 & 3.70 & 5.54 & 0.369 & 157.1 & 151 \\
\hline $\mathrm{Cu}$ & 50.9 & 0.9 & 0.95 & 9.33 & 4.28 & 6.39 & 0.426 & 161.1 & 157 \\
\hline $\mathrm{Cu}$. & 39.2 & 0.6 & 0.95 & 7.22 & 5.33 & 8.33 & $0.5 E 5$ & 162.5 & 157 \\
\hline $\mathrm{Cu}$. & 31.8 & 0.9 & 1.90 & 5.93 & 6.03 & 10.92 & 0.758 & 175.0 & 169 \\
\hline 7.n & 38.5 & 0.6 & 0.95 & 7.09 & 4.32 & 9.43 & 0.659 & 1.80 .7 & 175 \\
\hline
\end{tabular}

a U(93.3) plate at base of stack (sandwiches inverted) 


\section{TABLE IIA2}

U(93.3) METAL CYLINDERS DILUTED WITH MO, Ta, OR W, 15" DIAMETER, UNREFLECTED

Thickness of $U(93.3)$ plates $0.3 \mathrm{~cm}$

$U$ plate at base, portion of sandwich at top Average composition is that of final stack

Corrected for influence of supports of split stack

Communicated by G. A. Jarvis

\begin{tabular}{|c|c|c|c|}
\hline & vol \% & & $\begin{array}{l}\text { yers, } \\
\text { (cm) }\end{array}$ \\
\hline diluent $(A)$ & $\mathrm{U}(93 . \tilde{3})$ & $0(93.3)$ & $\bar{A}$ \\
\hline Mo & 89.2 & 0.6 & 0.08 \\
\hline Mo & 79.1 & 0.3 & 0.08 \\
\hline $\mathbf{T a}$ & 74.5 & 0.3 & 0.1 \\
\hline Ta & 59.4 & 0.3 & 0.2 \\
\hline Ta & 49.2 & 0.3 & 0.3 \\
\hline$w$ & 73.1 & 0.3 & 0.1 \\
\hline$\mapsto$ & 57.4 & 0.3 & 0.2 \\
\hline w & 47.3 & 0.3 & 0.3 \\
\hline$w$ & 40.2 & 0.3 & 0.4 \\
\hline$w$ & 35.0 & 0.3 & 0.5 \\
\hline 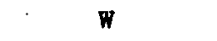 & 30.9 & 0.3 & 0.6 \\
\hline
\end{tabular}

\begin{tabular}{r}
$\begin{array}{c}\bar{p}(\mathrm{U}) \\
\left(\mathrm{g} / \mathrm{cm}^{3}\right)\end{array}$ \\
\hline 16.18 \\
15.15 \\
13.43 \\
10.77 \\
8.94 \\
13.21 \\
10.40 \\
8.63 \\
7.29 \\
6.46 \\
5.64
\end{tabular}

\begin{tabular}{c}
$\begin{array}{c}\bar{p}(\mathrm{~A}) \\
\left(\mathrm{g} / \mathrm{cm}^{3}\right)\end{array}$ \\
\hline 1.08 \\
2.09 \\
4.08 \\
6.52 \\
8.16 \\
4.92 \\
7.83 \\
9.72 \\
10.99 \\
12.15 \\
12.80
\end{tabular}

\begin{tabular}{c}
$\begin{array}{c}\mathrm{h}_{\mathrm{c}} \\
(\text { in.) }\end{array}$ \\
\hline 3.56 \\
3.96 \\
4.43 \\
5.73 \\
7.31 \\
4.33 \\
5.55 \\
6.74 \\
8.31 \\
9.85 \\
12.23
\end{tabular}

$\frac{h_{c} / d}{0.238}$

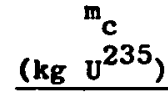

155.8

$m_{\max }$

0.238
0.264

155.1

151

0.295

160.7

166.7

0.382

0.487

176.5

154.4

155.8 .

0.370

0.480

157.2

163.7

$\begin{array}{lll}0.656 & 171.9 & 169\end{array}$

186.5 .

$\cdot 151$

151

157

169

151

151

151

157

181 


\section{TABLE IIA3}

U(93.3) METAL CTLINDERS DILUTED WITH Al, $\mathrm{Zr}$, OR Hf, 15" DIAMETER, UNREFLECTED

Thickness of $\mathrm{U}(93.3)$ plates $0.3 \mathrm{~cm}$

$U$ plate at base, portion of sandwich at top

Average composition is that of final stack

Corrected for influence of supports of split stack

Communicated by G. A. Jarvis

\begin{tabular}{|c|c|c|c|c|c|c|c|c|c|}
\hline diluent (A) & $\begin{array}{r}\text { vol } \% \\
\mathrm{u}(93.3) \\
\end{array}$ & $\begin{array}{c}\text { repeate } \\
\text { thickn } \\
\text { o(93.3) }\end{array}$ & $\begin{array}{c}\begin{array}{c}\text { ayers, } \\
(\mathrm{cm})\end{array} \\
A \\
\end{array}$ & $\begin{array}{c}\bar{\rho}(\mathrm{U}) \\
\left(\mathrm{g} / \mathrm{cm}^{3}\right) \\
\end{array}$ & $\begin{array}{r}\bar{p}(\mathrm{~A}) \\
\left(\mathrm{g} / \mathrm{cm}^{3}\right) \\
\end{array}$ & $\begin{array}{c}h_{c} \\
(i n .) \\
\end{array}$ & $\mathrm{h}_{\mathrm{c}} / \mathrm{d}$ & $\left(\begin{array}{c}\mathrm{m}_{\mathrm{c}} \\
\left(\mathrm{kg} \mathrm{\textrm {U } ^ { 2 3 5 }}\right) \\
\end{array}\right.$ & $\left(\begin{array}{r}\left.\max _{\max } \mathrm{v}^{235}\right) \\
\end{array}\right.$ \\
\hline AI & 78.6 & 0.3 & 0.08 & 14.20 & 0.555 & 4.14 & 0.276 & 159.0 & 157 \\
\hline Al & 64.8 & 0.3 & 0.16 & 11.68 & 0.912 & 5.22 & 0.348 & 164.9 & 163 \\
\hline Al & 55.2 & 0.3 & 0.24 & 9.97 & 1.166 & 6.39 & 0.426 & 172.2 & 169 \\
\hline Al & 48.0 & 0.3 & 0.32 & 8.70 & 1.358 & 7.67 & 0.512 & 180.4 & 175 \\
\hline Al & 42.6 & 0.3 & 0.40 & 7.75 & 1.502 & 9.23 & 0.615 & 193.2 & 187 \\
\hline $\mathrm{zr}$ & 71.3 & 0.3 & 0.1 & 13.18 & 1.810 & 4.44 & 0.296 & 158.2 & 157 \\
\hline $\mathbf{z r}$ & 56.6 & 0.3 & 0.2 & 10.31 & 2.70 & 5.84 & 0.389 & 162.5 & 157 \\
\hline $\mathrm{Zr}$ & 46.5 & 0.3 & 0.3 & 8.45 & 3.32 & 7.42 & 0.495 & 169.4 & 163 \\
\hline $\mathbf{z r}$ & 39.6 & 0.3 & 0.4 & 7.18 & 3.74 & 9.22 & 0.614 & 178.7 & 175 \\
\hline Hf & 97.3 & $\left(\begin{array}{l}3.6 \\
3.9\end{array}\right)^{a}$ & 0.1 & 17.55 & 0.349 & 3.27 & 0.218 & 155.1 & 151 \\
\hline Hf & 93.4 & 1.5 & 0.1 & 16.80 & 0.837 & 3.45 & 0.230 & 156.6 & 151 \\
\hline Hf & 8.5 .1 & 0.6 & 0.1 & 15.31 & 1.904 & 3.82 & 0.255 & 158.1 & 157 \\
\hline Hf & 74.1 & 0.3 & 0.1 & 13.30 & 3.30 & 4.48 & 0.299 & 161.2 & 157 \\
\hline
\end{tabular}

a The two thicknesses of $U$ alternate in successive sandwiches 


\section{TABLE IIBI}

U(93.3) - GRAPHITE CYLINDERS, UNREFLECTED

Thlckness of $U(93.3)$ plates $0.3 \mathrm{~cm}$

U plate at base, portion of sandwich at top

Average composition is that of final stack

Corrected for influence of supports of split stack

Communicated by G. A. Jarvis

\begin{tabular}{|c|c|c|}
\hline \multirow{2}{*}{$\begin{array}{r}\operatorname{vol} \% \\
\text { U(93.3) } \\
\end{array}$} & \multicolumn{2}{|c|}{$\begin{array}{l}\text { repeated layers, } \\
\text { thickness }(\mathrm{cm})\end{array}$} \\
\hline & & graphite \\
\hline \multicolumn{3}{|c|}{ 15" diameter cylinders: } \\
\hline 86.0 & 2.4 & 0.40 \\
\hline 82.2 & 1.8 & 0.40 \\
\hline 79.2 & 1.5 & 0.40 \\
\hline 75.5 & 1.2 & 0.40 \\
\hline 69.7 & 0.9 & 0.40 \\
\hline 60.7 & 0.6 & 0.40 \\
\hline 53.6 & 0.9 & 0.80 \\
\hline 43.5 & 0.3 & 0.40 \\
\hline 33.8 & 0.6 & 1.20 \\
\hline 28.0 & 0.3 & 0.80 \\
\hline
\end{tabular}

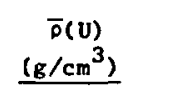

$\bar{p}(\mathrm{C})$ $\underline{\left(\mathrm{g} / \mathrm{cm}^{3}\right)}$

15.56

$14.78 \quad 0.282$

$14.28 \quad 0.330$

$13.97 \quad 0.399$

$12.70 \quad 0.485$

11.06

9.97

8.07

6.22

5.26

0.631

0.758

0.921

1.075

1.188

8.94

$\begin{array}{lll}47.7 & 0.3 & 0.32\end{array}$

$\begin{array}{lll}31.8 & 0.3 & 0.64\end{array}$

$23.7 \quad 0.3$

$19.00 \quad 0.3$

$15.79 \quad 0.3$

$13.53 \cdot 0.3$

$11.89-0.3$

10.470 .3

9.44

0.3

0.95

1.27

1.59

1.90

2.22

2.54

2.86

$2.93 \quad 0.021$ av 0.71

5.97

4.44

3.56

2.96

2.54

2.23

1.965

0.830

1.123

1.248

1.345

1.392

1.434

1.467

1.540

1.551

$1(0.002)$

0.539

, 573

$573 \quad 35.9$

$35: 9$

$\begin{array}{ll}5.46 & 0.260 \\ 7.81 & 0.372\end{array}$

$10.48 \quad 0.499$

$13.08 \quad 0.623$

$16.49 \quad 0.785$

20.1

0.958

$23.9 \quad 1.138$

$28.3 \quad 1.345$

1.734

0.0083 .0 .0076 av 0.74

U(93.15) 1011

48.0

$.004^{\prime \prime}$

.

$\begin{array}{ll}267 & 259 \\ 260 & 247 \\ 258 & 247 \\ 258 & 247 \\ 263 & 259 \\ 274 & 270 \\ 292 & 282 \\ 303 & 294 \\ 350 & 341\end{array}$

$\dot{m}_{\max }$

$\mathrm{kg} \mathrm{u^{235 } )}$

$\begin{array}{ll}154.5 & 145 \\ 155.0 & 145 \\ 154.7 & 150 \\ 156.3 & 150 \\ 156.0 & 145 \\ 156.5 & 150 \\ 157.7 & 153 \\ 163.1 & 157 \\ 172.3 & 169 \\ 193.6 & 187\end{array}$

303

285

(58):

critical. 
TABLE II IB2a

GRAPHITE-MODERATED HIGHLY-ENRI GHED U, REFLECTOR OF GRAPHITE

\begin{tabular}{|c|c|c|c|c|c|c|c|c|c|c|c|}
\hline ref & \multicolumn{6}{|c|}{ core } & \multicolumn{3}{|c|}{ reflector } & $\underline{\left(\mathrm{kg} \mathrm{\textrm {U } ^ { 2 3 5 }}\right)}$ & $\begin{array}{r}m_{\max } \\
\left(\mathrm{kg} \mathrm{v} \mathrm{v}^{235}\right) \\
\end{array}$ \\
\hline (22) & $\begin{array}{l}21.00 d 1 a \\
h_{c} / d=0.112\end{array}$ & $\begin{array}{l}U(93.3) \\
(47.7 v / 0)\end{array}$ & $0.30 \mathrm{~cm}$ & $0.32 \mathrm{~cm}$ & 8.94 & 0.83 & $\begin{array}{l}\text { both planes } \\
\left(21.0^{\prime \prime} \text { dia) }\right.\end{array}$ & 6.00 & 1.7 & $112.1^{\mathrm{a}}$ & 105.8 \\
\hline (22) & $\begin{array}{l}21.00 d 1 a \\
h_{c} / d-0.373\end{array}$ & $\begin{array}{l}U(93.3) \\
\left(19.0^{\circ} v / 0\right)\end{array}$ & $0.30 \mathrm{~cm}$ & $1.27 \mathrm{~cm}$ & 3.56 & 1.34 & $\begin{array}{l}\text { both planes } \\
\text { (21.0" d1a) }\end{array}$ & 6.00 & 1.7 & $148.4^{\mathrm{a}}$ & 141.1 \\
\hline (22) & $\begin{array}{l}21.00 \text { dia } \\
n_{c} / d-1.04\end{array}$ & $\begin{array}{l}\mathrm{U}(93.3) \\
(10.47 \mathrm{v} / 0)\end{array}$ & $0.35 \mathrm{~cm}$ & $2.54 \mathrm{~cm}$ & 1.96 & 1.54 & $\begin{array}{l}\text { both planes } \\
\left(21.0^{+1} d^{\prime} a\right)\end{array}$ & 6.00 & 1.7 & $228.5^{a}$ & 222.7 \\
\hline (22) & $\begin{array}{l}21.00 d 1 a \\
h_{c} / d-1.3 s\end{array}$ & $\begin{array}{l}U(93,3) \\
(9.44 \mathrm{v} / 0)\end{array}$ & $0.30 \mathrm{~cm}$ & $2.86 \mathrm{~cm}$ & 1.77 & 1.55 & $\begin{array}{l}\text { both planes } \\
\left(21.0^{p} \text { dia) }\right.\end{array}$ & 6.00 & 1.7 & $276.0^{\mathrm{a}}$ & 258.6 \\
\hline (22) & $\begin{array}{l}21.00 d 1 a \\
h_{c} / d-2.10\end{array}$ & $\begin{array}{l}u(93.3) \\
(8.76 \mathrm{v} / 0)\end{array}$ & $0.30 \mathrm{~cm}$ & $3.18 \mathrm{~cm}$ & 1.62 & 1.57 & $\begin{array}{l}\text { both planes } \\
\left(21.0^{\prime \prime} \text { dxa) }\right.\end{array}$ & 6.00 & 1.7 & $377.6^{\mathrm{a}}$ & 364.4 \\
\hline (32) & $\begin{array}{l}40.0 \times 36.5 \\
\times 36.0\end{array}$ & $U(93.2)$ & $0.001 "$ & $\left(c / v^{235}\right.$ & - 3369) & 1.50 & complete & 12.00 & 1.55 & $7.38^{c}$ & critical \\
\hline (32) & $\begin{array}{l}40.0 \times 33.0 \\
\times 33.0\end{array}$ & $U(93.2)$ & $0.001 "$ & $\left(c, u^{235}\right.$ & $-2538)$ & 1.50 & complete & 12.00 & 1.55 & $8.24^{c}$ & crittcal \\
\hline (32) & $\begin{array}{l}48.0 \times{ }^{48.0} \\
\times 45.6 \mathrm{~d}^{4}\end{array}$ & $U(93.2)$ & 0.301" & $\left(c / v^{235}\right.$ & - 4685) & 1.34 & complete & 12.00 & 1.55 & $9.07^{c}$ & crittcal \\
\hline (32) & $\begin{array}{l}42.0 \times 39.0 \\
\times 40.0\end{array}$ & $U(93.2)$ & $0.001 "$ & $\left(\mathrm{c} / \mathrm{v}^{235}\right.$ & $-2972)$ & 1.34 & complete & 12.00 & 1.55 & $9.52^{c}$ & critical \\
\hline
\end{tabular}

" No correction for 0.020 " thick ss dipphragm across median plane of assexbly

b Enpirical correction for 0.063 " ss diaphragm supporting part of assembly

c Core and raflector contaln $0.061 \mathrm{~V} / \mathrm{C}$. $\mathrm{Al}$ (1100F) as a atrix of 3" square tubes

d Three extra 3" square tubes are averaged into this dimension of core 


\section{TABLE IIB2b}

GRAPHITE-HODERATED HIGHY-ENRICHED U, REFLECTOR OF Be (PLUS SOME GRAPHITE)

Core and reflector contain $A 1(1100)$ at $\bar{p}-0.165 \mathrm{~g} / \mathrm{cm}^{3}$ as matrix of $3^{\prime \prime}$ square tubes; forms are pseudocylinders One-inch thick unloaded graphite across face 1 of core

Core uranium is $\mathrm{U}(93.2) ; \overline{\mathrm{p}}(\mathrm{Be})=1.66 \mathrm{~g} / \mathrm{cm}^{3}$

$U$ foil in core 0.002 " thick up to $16.3 \mathrm{~kg} \mathrm{U}^{235}$, beyond which 0.005 " thick foll intermixed

All assemblies critical

\begin{tabular}{|c|c|c|c|c|c|c|c|c|c|}
\hline \multirow[b]{2}{*}{ ref } & \multicolumn{3}{|c|}{ Core } & \multicolumn{5}{|c|}{ reflector } & \multirow[b]{2}{*}{$\begin{array}{r}\begin{array}{r}\mathrm{m}_{\mathrm{c}} \\
\left(\mathrm{kg} \mathrm{v}^{235}\right)\end{array} \\
\end{array}$} \\
\hline & $\begin{array}{c}\text { dimensions } \\
\text { (in.) }\end{array}$ & $\mathrm{c} / \mathrm{u}^{235}$ & $\begin{array}{c}\bar{p}(\mathrm{C}) \\
\left(\mathrm{g} / \mathrm{cm}^{3}\right) \\
\end{array}$ & & $\begin{array}{l}\text { Be thickness } \\
\text { waII }\end{array}$ & $\begin{array}{l}\text { (in.) } \\
\text { face I }\end{array}$ & tace 2 & $\begin{array}{l}\text { graphite thickness } \\
\text { against core } \\
\text { face } 2 \text { (in.) } \\
\end{array}$ & \\
\hline (33) & 24.6 av dia $\times 30.6$ & 125 & 1.42 & $.4 .88 \mathrm{av}$ & & 4.00 & 8.00 & 0.40 & 53.6 \\
\hline (33) & 24.6 av dia $\times 30.6$ & 125 & 1.42 & $5.39 \mathrm{av}$ & & 4.00 & 4.00 & 0.40 & 53.6 \\
\hline (33) & 31.9 av dia $\times 31.0$ & 395 & 1.42 & $5.10 \mathrm{av}$ & & 4.50 & 3.20 & 0.00 & 28.7 \\
\hline (33) & 36.3 av dia $\times 31.0$ & 395 & 1.29 & $5.51 \mathrm{av}$ & . & $\begin{array}{l}3.00 \\
(1.27 " \mathrm{C} \\
\text { faces of }\end{array}$ & $\begin{array}{l}3.00 \\
\text { outside } \\
\text { Be) }\end{array}$ & 0.00 & 33.5 \\
\hline (33) & 38.4 av dia $\times 31.0$ & 398 & 1.30 & $5.04 \mathrm{av}$ & & 3.35 & 3.35 & 0.00 & 37.6 \\
\hline (33) & 38.4 av dia $\times 30.0$ & 1022 & 1.48 & 4.72 av & & 3.85 & $\begin{array}{l}0.00 \\
9.1 \mathrm{C}\end{array}$ & 1.00 & 16.2 \\
\hline (33) & 38.4 av dia $\times 30.0$ & 1022 & 1.48 & $4.72 \mathrm{av}$ & & 3.85 & 3.85 & 1.00 & 16.2 \\
\hline (34) & 55.0 av dia $\times 52.0$ & 1350 & 1.17 & $\begin{array}{l}\text { Inside: } \\
\text { outside: }\end{array}$ & $\begin{array}{l}C, 2.00 \mathrm{av} \\
\mathrm{Be}, 6.50 \mathrm{av}\end{array}$ & $\begin{array}{l}0.00 \\
\text { (no C on }\end{array}$ & $\begin{array}{r}0.00 \\
\text { face) }\end{array}$ & 0.00 & 32.0 \\
\hline
\end{tabular}




\section{TABLE IICl}

LUCITE MODERATED U(93.16), UNREFLECTED

Al matrix throughout core and as incidental reflector, $\bar{p}$ (Al) $=0.165 \mathrm{~g} / \mathrm{cm}^{3}$

Probable error in $m_{c}$ about $\pm 1 \%$ for critical systems

Communicated by J. C. Hoogterp

\begin{tabular}{|c|c|c|c|c|c|c|}
\hline $\begin{array}{l}\text { thicknesses of } \\
\text { alternating layers (in.) }\end{array}$ & $\begin{array}{r}\bar{p}\left(\mathrm{u}^{235}\right) \\
\left(\mathrm{g} / \mathrm{cm}^{3}\right) \\
\end{array}$ & $\frac{\text { av atom }}{\mathrm{H} / \mathrm{U}^{235}}$ & $\frac{\text { c ratio }}{c / 0^{235}}$ & $\begin{array}{l}\text { dimensicns } \\
\text { (1n.) }\end{array}$ & 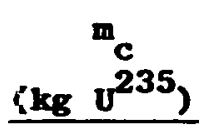 & $M_{\max }$ \\
\hline $0.012 \mathrm{U}, 1 / 16$ lucite & 2.12 & 5.99 & 3.74 & $23.5 \times 12 \times 10.87$ & 106.5 & critical \\
\hline $0.012 \mathrm{U}, 1 / 16$ luc1te & 2.31 & 5.99 & $\mathbf{3 . 7 4}$ & $23.5 \times 12 \times 9.26$ & 98.8 & critical \\
\hline $0.006 \mathrm{U}, 1 / 16$ lucite & 1.317 & 12.12 & 7.57 & $15 \times 11.4 \times 12$ & 44.2 & critical \\
\hline $0.002 \mathrm{U}, 1 / 16$ lucite & 0.491 & 35.4 & 22.1 & $15 \times 12 \times 11.46$ & 16.61 & critical \\
\hline $0.004 \mathrm{U}, 1 / 8$ lucite & 0.476 & 35.6 & 22.3 & $15 \times 12 \times 12$ & 16.83 & 128 \\
\hline $0.008 \mathrm{v}, 1 / 4$ lucite & 0.477 & 35.4 & 22.1 & $15 \times 12 \times 12$ & 16.89 & critical \\
\hline $0.012 \mathrm{U}, 3 / 8$ lucite & 0.489 & 35.3 & 22.2 & $15 \times 12 \times 11.65$ & 16.78 & critical \\
\hline $0.016 \mathrm{U}, 1 / 2$ lucite & 0.484 & 35.2 & 22.0 & $15 \times 12 \times 12.06$ & 17.22 & critical \\
\hline $0.022 \mathrm{U}, 11 / 16$ lucite & 0.494 & 35.1 & 22.0 & $15 \times 12 \times 12$ & 17.48 & critical \\
\hline $0.030 \mathrm{v}, 15 / 16$ lucite & 0.495 & 35.1 & 22.0 & $15 \times 12 \times 12.47$ & 18.22 & critical \\
\hline
\end{tabular}


TABLE IIC2a

LUCITE MODERATED U(93.16), $\geq 6$ " THICK LUCITE REFLECTOR

Al matrix throughout core and reflector, $\bar{n}(\mathrm{Al})-0.165 \mathrm{~g} / \mathrm{cm}^{3}$

Probable error in $m_{c}$ about $\pm 1 \%$ for critical systems

Communicated by J. C. Hoogterp

\begin{tabular}{|c|c|c|c|c|c|c|c|c|}
\hline \multicolumn{5}{|c|}{ core } & \multicolumn{2}{|c|}{ reflector } & \multirow[b]{2}{*}{$\left(\mathrm{kg} \mathrm{u}^{\mathrm{m}_{\mathrm{c}}} \mathrm{u}^{235}\right)$} & \multirow[b]{2}{*}{$n_{\max }$} \\
\hline $\begin{array}{l}\text { thicknesses of } \\
\text { alternating layers (in.) }\end{array}$ & $\begin{array}{l}\overline{0}\left(\mathrm{u}^{235}\right) \\
\left(\mathrm{g} / \mathrm{cm}^{3}\right) \\
\end{array}$ & $\frac{\text { av atoon } 1}{\mathrm{H} / \mathrm{U}^{235}}$ & $\frac{c \text { ratto }}{c / u^{235}}$ & $\begin{array}{l}\text { critical size } \\
(\mathrm{L} \times \mathrm{H} \times \mathrm{W}-\mathrm{in.}) \\
\end{array}$ & $\begin{array}{c}\bar{p} \text { (lucite) } \\
\left(\mathrm{g} / \mathrm{cm}^{3}\right) \\
\end{array}$ & $\begin{array}{l}\text { thickness/face } \\
\left(\mathrm{L}^{\mathrm{a}} \times \mathrm{B} \times \mathrm{W}-1 \mathrm{n} .\right) \\
\end{array}$ & & \\
\hline $0.006 \mathrm{U}, 1 / 16$ lucite & 1.311 & 12.2 & 7.6 & $15 \times 6 \times 8.23$ & 1.007 & $8.25 \times\left(\begin{array}{l}6 \\
9\end{array}\right) \times 9$ & 15.90 & critical \\
\hline $0.006 \mathrm{v}, 1 / 16$ lucite & 1.213 & 13.3 & 8.3 & $15 \times 6 \times 8$ & 1.048 & $8.25 \times 6 \times 6$ & 14.32 & critical \\
\hline $0.006 \mathrm{U}, 1 / 16$ lucite & 1.109 & 12.1 & 7.6 & $15 \times 9 \times 7.5$ & 1.037 & $8.25 \times 6 \times 6$ & 18.41 & critical \\
\hline $0.006 \mathrm{U}, 1 / 16$ lucite & 0.950 & 12.0 & 7.5 & $15 \times 9 \times 10.5$ & 1.036 & B.25 $\times 6 \times 6$ & 22.06 & critical \\
\hline $0.002 \mathrm{U}, 1 / 16$ lucite & 0.363 & 35.6 & 22.3 & $15 \times 9 \times 10.5$ & 1.036 & $8.25 \times 6 \times 6$ & 8.43 & critical \\
\hline $0.002 \mathrm{U}, 1 / 16$ lucite & 0.452 & 35.3 & 22.0 & $15 \times 6 \times 10.5$ & 1.044 & $8.25 \times 6 \times 6$ & 7.00 & critical \\
\hline $0.002 \mathrm{v}, 1 / 16$ lucite & 0.517 & 34.3 & 21.5 & $15 \times 6 \times 8$ & 1.040 & $8.25 \times 6 \times 6$ & 6.10 & 254 \\
\hline $0.004 \mathrm{U}, 1 / 8$ lucite & 0.518 & 34.3 & 21.5 & $15 \times 6 \times 8.03$ & 1.040 & $8.25 \times 6 \times 6$ & 6.14 & 725 \\
\hline $0.008 \mathrm{U}, 1 / 4$ lucite & 0.518 & 34.3 & 21.4 & $15 \times 6 \times 8.10$ & 1.048 & $8.25 \times 6 \times 6$ & 6.19 & critical \\
\hline $0.012 \mathrm{U}, 3 / 8$ lucite & 0.518 & 34.3 & 21.5 & $15 \times 6 \times 8.13$ & 1.048 & $8.25 \times 6 \times 6.12$ & 6.21 & critical \\
\hline $0.016 \mathrm{v}, 1 / 2$ lucite & 0.521 & 34.3 & 21.5 & $15 \times 6 \times 8.39$ & 1.050 & $8.25 \times 6 \times 6.06$ & 6.45 & critical \\
\hline $0.024 \mathrm{v}, 3 / 4$ lucite & 0.532 & 33.5 & 20.9 & $15 \times 6 \times 9.11$ & 1.035 & $8.25 \times 6 \times 6.06$ & 7.14 & critical \\
\hline $0.030 \mathrm{U}, 15 / 16$ lucite & 0.509 & 35.3 & 22.0 & $15 \times 6 \times 10.26$ & 1.031 & $8.25 \times 6 \times 6.09$ & 7.70 & critical \\
\hline $0.030 \mathrm{U}, 13 / 16$ lucite & 0.582 & 30.6 & 19.1 & $15 \times 6 \times 9.48$ & 1.031 & $8.25 \times 6 \times 6.26$ & 8.13 & 519 \\
\hline $0.030 \mathrm{~V}, 11 / 16$ lucite & 0.685 & 26.0 & 16.2 & $15 \times 6 \times 8.04$ & 1.030 & $8.25 \times 6 \times 6.98$ & 8.12 & 893 \\
\hline $0.030 \mathrm{v}, 9 / 16$ lucite & 0.818 & 21.2 & 13.2 & $15 \times 6 \times 8.34$ & 1.031 & $8.25 \times 6 \times 6.83$ & 10.06 & critical \\
\hline $0.030 \mathrm{v}, 7 / 16$ lucite & 1.021 & 16.6 & 10.4 & $15 \times 6 \times 7.89$ & 1.030 & $8.25 \times 6 \times 7.05$ & 11.89 & 170 \\
\hline $0.008 \mathrm{~J}, 15 / 16$ lucite & 0.138 & 133.3 & 83.2 & $15 \times 6 \times 17.09$ & 1.001 & $8.25 \times 6 \times 6.04$ & 3.47 & critical \\
\hline $0.008 \mathrm{v}, 11 / 16$ lucite & 0.186 & 97.8 & 61.1 & $15 \times 6 \times 12.35$ & 1.000 & $8.25 \times 6 \times 8.33$ & 3.38 & critical \\
\hline $0.008 \mathrm{v}, 9 / 16$ lucite & 0.229 & 79.4 & 49.6 & $15 \times 6 \times 10.79$ & 1.000 & $8.25 \times 6 \times 9.11$ & 3.65 & 28 \\
\hline
\end{tabular}

a Reflector thickness on ends averaged to allow for 1/2" irregularity 
TABLE IIC2b

LUCITE MODERATED U(93.16) SLABS, 6" THICK LUCITE REFLECTORS ON TWO LARGE SURFACES ONLY

Alternating layers of $0.002 " U$ and $1 / 16$ " lucite in core

Al matrix throughout core and reflector, $\bar{\rho}$ (Al) $-0.165 \mathrm{~g} / \mathrm{cm}^{3}$

Probable error in $m_{c}$ about $\pm 1 \%$ for critical systems

Communicated by J. C. Hoogte=p

\begin{tabular}{|c|c|c|c|c|c|c|}
\hline \multirow{2}{*}{ 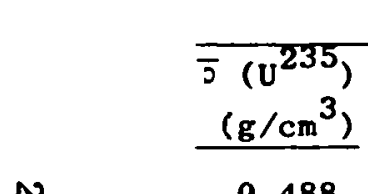 } & \multicolumn{3}{|c|}{ core } & reflector & \multirow[b]{2}{*}{  } & \multirow[b]{2}{*}{$M_{\max }$} \\
\hline & $\begin{array}{l}\text { av a tomi } \\
\mathrm{H} / \mathrm{U}^{235} \\
\end{array}$ & $\begin{array}{c}\text { c rat1o } \\
\mathrm{c} / \mathrm{U}^{235} \\
\end{array}$ & $\begin{array}{c}\text { dimensions } \\
(\text { in.) }\end{array}$ & $\begin{array}{c}\bar{p}(\text { lucite }) \\
\left(\mathrm{g} / \mathrm{cm}^{3}\right) \\
\end{array}$ & & \\
\hline $\begin{array}{ll}N & 0.488\end{array}$ & 35.3 & 22.1 & $15^{a} \times 6 \times 14.53$ & 0.977 & 10.45 & 2320 \\
\hline 0.422 & 36.0 & 22.5 & $32 \times 6 \times 13.06$ & 1.007 & 17.34 & critical \\
\hline 0.372 & 35.7 & 22.3 & $32 \times 6 \times 17.03$ & 1.006 & 19.96 & 2320 \\
\hline 0.478 & 35.0 & 22.5 & $32 \times 5.24 \times 12$ & 1.022 & 15.79 & 102 \\
\hline 0.491 & 35.9 & 22.4 & $32 \times 5 \times 12$ & 1.022 & 15.44 & 1821 \\
\hline 0.479 & 37.3 & 23.3 & $32 \times 3 \times 23.03$ & 1.041 & 17.37 & critical \\
\hline 0.479 & 35.6 & 22.2 & $32 \times 3 \times 28.5$ & 1.002 & 21.5 & critical \\
\hline 0.431 & 35.7 & 22.3 & $32 \times 3 \times 54$ & 1.041 & 36.6 & critical \\
\hline 0.474 & 36.2 & 22.6 & $32 \times 2.71 \times 48$ & 1.040 & 32.4 & 187 \\
\hline 0.473 & 36.1 & 22.6 & $32 \times 2.69 \times 52.8$ & 1.037 & 35.2 & critical \\
\hline 0.498 & 36.2 & 22.6 & $32 \times 2.50 \times 58.9$ & 1.041 & 38.5 & 38.6 \\
\hline
\end{tabular}

a Reflector overhangs fuel $1 / 2$ " on both sides 


\section{TABLE IIC3}

POLYETHYLENE-MODERATED U(93.15) PARALLELEPIPEDS, BERYLLIUM REFLECTOR

CORE: U(93.15) foil interleaved with polyethylene slabs Reflector: Brush QMV S-200-C beryllium, $\bar{\rho}=1.85 \mathrm{~g} / \mathrm{cm}^{3}$

Reference (59)

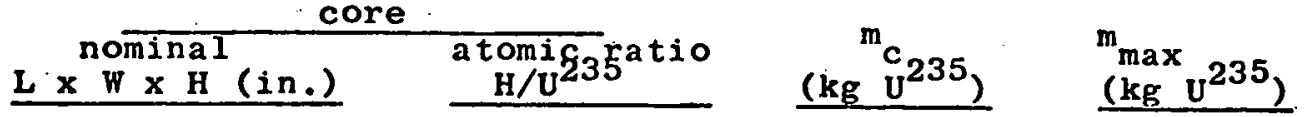

Be reflector 12.5 " thick, 0.0012 " thick $U$, polyethylene $\bar{\rho}=0.961 \mathrm{~g} / \mathrm{cm}^{3}$ :

$\begin{array}{rllll}6.0 \times 6.125 \times 5.75 & 375^{\mathrm{a}} & 0.299 & 0.291 \\ 6.0 \times 6.125 \times 4.75 & 316^{\mathrm{a}} & 0.292 & 0.291 \\ 6.0 \times 6.125 \times 3.75 & 242^{\mathrm{a}} & 0.301 & 0.297\end{array}$

Be reflector $12.0^{\prime \prime}$ thick, 0.0012 " thick $U$, polyethylene $\bar{p}=0.947 \mathrm{~g} / \mathrm{cm}^{3}$ :

$6.5 \times 6.625 \times 4.75 \quad 340 \quad 0.313 \quad 0.311$

Be reflector 11.5" thick, $0.0020^{\prime \prime}$ thick $U$, polyethylene $\bar{\rho}=0.883 \mathrm{~g} / \mathrm{cm}^{3}$ :

$\begin{array}{llll}8.0 \times 8.125 \times 7.75 & 540 & 0.456 & 0.451 \\ 8.0 \times 8.125 \times 6.50 & 489 & 0.422 & 0.414 \\ 8.0 \times 8.125 \times 5.00 & 411 & 0.386 & 0.376 \\ 8.0 \times 8.125 \times 3.63 & 318 & 0.360 & 0.339 \\ 8.0 \times 8.125 \times 2.75 & 248 & 0.352 & 0.339 \\ 8.0 \times 8.125 \times 2.25 & 190 & 0.376 & 0.339\end{array}$

${ }^{a} \mathrm{U}$ foll on all six sides of core 


\section{TABLE IIDI}

LUCITE-GRAPHITE MODERATED U(93.16), UNREFLECTED

Lucite thickness 1/16" per indicated thicknesses of $U$ and graphite

Al matrix throughout core and as incidental reflector, $\bar{p}(\mathrm{Al})=0.165 \mathrm{~g} / \mathrm{cm}^{3}$

Probable error in $m_{c}$ about $\pm 1 \%$ for critical systems

Communicated by J. C. Hoogterp

\begin{tabular}{|c|c|c|c|c|c|c|c|}
\hline & $\begin{array}{l}\text { thicknesses of layers } \\
\text { with } 1 / 16^{\prime \prime} \text { lucite (1n.) } \\
\end{array}$ & $\begin{array}{l}\bar{F}\left(\mathrm{U}^{235}\right) \\
\left(\mathrm{g} / \mathrm{cm}^{3}\right) \\
\end{array}$ & $\frac{\text { av a ton }}{\mathrm{H} / \mathrm{U}^{235}}$ & $\frac{\mathrm{cratio}}{\mathrm{c} / \mathrm{v}^{235}}$ & $\begin{array}{l}\text { dimensions } \\
\text { (in.) }\end{array}$ & $\left(\begin{array}{c}\left.\mathrm{kg} \mathrm{v}_{\mathrm{c}} \mathrm{u}^{235}\right) \\
\end{array}\right.$ & $\mathrm{u}_{\max }$ \\
\hline & $0.012 \mathrm{U}, 0.120$ graphite & 0.916 & 6.02 & 24.1 & $23.5 \times 18.14 \times 18$ & 115.2 & critical \\
\hline & $0.012 \mathrm{U}, 0.280$ graphite & 0.518 & 6.04 & 48.7 & $23.5 \times 28.02 \times 24$ & 134.2 & 317 \\
\hline & $0.006 \mathrm{U}, 0.280$ graphite & 0.258 & 12.41 & 98.2 & $32 \times 24.70 \times 24.70$ & 82.4 & critical \\
\hline & $0.006 \mathrm{U}, 0.280$ graphite & 0.258 & 12.41 & 98.2 & $32 \times 13.61$ av radius ${ }^{2}$ & 78.6 & critical \\
\hline & 0.006 U: 0.280 graphite & 0.258 & 12.27 & 98.7 & $23.5 \times 28.5 \times 28.79$ & 81.4 & critical \\
\hline & $0.002 \mathrm{U}, 0.120$ graphite & 0.337 & 35.2 & 51.8 & $15 \times 15 \times 15.69$ & 19.51 & critical \\
\hline & $0.002 \mathrm{U}, 0.120$ graphite & 0.337 & 35.2 & 48.2 & $15 \times 15 \times 16.69$ & 20.7 & critical \\
\hline & $0.002 U, 0.280$ graphite & 0.224 & 35.0 & 101.5 & $15 \times 21 \times 21$ & 24.2 & critical \\
\hline
\end{tabular}

a Pseudocylinder with 3" nodule 
TABLE IID2a

LUCITE-GRAPHITE MODERATED U(93.16), LUCITE REFLECTED

U thickness 0.006 " and lucte thickness $1 / 16$ " per indicated thickness of graphite in core

Al matrix throughout core and reflector, $\bar{n}(A 1)=0.165 \mathrm{~g} / \mathrm{cm}^{3}$

All systems critical, probable error in $m_{c}$ about $\pm 1 \%$

Communicated by J. C. Hoogterp

\begin{tabular}{|c|c|c|c|c|c|c|c|c|c|c|}
\hline \multicolumn{7}{|c|}{ core } & \multicolumn{3}{|c|}{ reflector } & \multirow[b]{2}{*}{$\left(\begin{array}{c}\left.\mathrm{kg} \mathrm{v}^{\mathrm{m}} \mathrm{u}^{235}\right) \\
\end{array}\right.$} \\
\hline $\begin{array}{l}\text { graphite } \\
\text { per } 0.006^{\prime \prime}\end{array}$ & $\begin{array}{l}\text { thickness } \\
\text { U, } 1 / 16^{" 1}\end{array}$ & $\begin{array}{l}\text { (in.) } \\
\text { lucite }\end{array}$ & $\begin{array}{l}\bar{D}\left(\mathrm{U}^{235}\right) \\
\left(\mathrm{g} / \mathrm{cm}^{3}\right) \\
\end{array}$ & $\frac{\text { av atom }}{H / v^{235}}$ & $\frac{\mathrm{rat10}}{\mathrm{c} / \mathrm{v}^{235}}$ & $\begin{array}{c}\text { critical size } \\
(L \times H \times W-i n .)\end{array}$ & $\frac{\overline{\bar{o} \text { (1uc1te) }}}{\text { ends }}$ & $\frac{\left(\mathrm{g} / \mathrm{cm}^{3}\right)}{\text { sides }}$ & $\begin{array}{l}\text { thrckness/face } \\
\left(\mathrm{L}^{\mathrm{a}} \mathrm{x} \text { H } \times \mathrm{W}-\mathrm{in} .\right) \\
\end{array}$ & \\
\hline & 0.280 & & 0.255 & 12.46 & 100.3 & $23.5 \times 24 \times 24$ & 1.021 & 0.938 & $.6 \times 1.50 \times 1.50$ & 43.2 \\
\hline & 0.280 & & 0.223 & 12.48 & 100.6 & $23.5 \times 21 \times 21$ & 1.021 & 0.969 & $26 \times 4 \times 4$ & 37.8 \\
\hline & 0.280 & & 0.252 & 12.12 & 101.2 & $23.5 \times 18 \times 18$ & 1.042 & 0.984 & $\sim 6 \times 3.75 \times 3.75$ & 31.5 \\
\hline & 0.280 & & 0.244 & 12.12 & 101.2 & $23.5 \times 18 \times 18$ & 1.042 & 1.021 & $.6 \times 7.50 \times 7.50$ & 30.5 \\
\hline & 0.280 & & 0.252 & 12.05 & 101.5 & $23.5 \times 17.25 \times 17.4$ & 1.042 & 0.982 & $.6 \times 6.38 \times 6.32$ & 29.0 \\
\hline & 0.280 & & 0.251 & 12.10 & 101.6 & $23.5 \times 9.57$ av radius ${ }^{b}$ & 1.042 & 0.980 & $\sim 6 \times 7.10 \mathrm{av}^{\mathrm{b}}$ & 27.8 \\
\hline & $0.004 \mathrm{av}$ & & 0.934 & 12.05 & 8.89 & $15 \times 9 \times 10.5$ & 1.036 & 1.036 & $8.25 \times 6 \times 6$ & 21.7 \\
\hline
\end{tabular}

a Low-density 1.5" extension of 6" thick reflector; 8.25" end reflector thickness is averaged over 1/2" irregularity

b Pseudocylinder wth 3" module

c Average of nonuniformly-distributed $0.120 "$-thick graphite 


\section{TABLE IID2b}

LUCITE-GRAPHITE MODERATED U(93.16) SLABS, 6" THICK LUCITE REFLETTORS ON TWO LARGE SURFA.CES ONLY

Core consists of the successive layers: $0.006^{\prime \prime} \mathrm{U}, 1 / 16^{\prime \prime}$ Iucite, $0.280^{\prime \prime}$ graphite Al matrix throughout core and reflector, $\bar{\rho}(A 1)=0.165 \mathrm{~g} / \mathrm{cm}^{3}$

All systems critical, probable error in $m_{c}$ about $\pm 1 \%$

Communicated by J. C. Hoogterp

core

\begin{tabular}{|c|c|c|c|c|}
\hline $\begin{array}{l}f\left(\mathrm{U}^{235}\right) \\
\left(\mathrm{g} / \mathrm{cm}^{3}\right) \\
\end{array}$ & $\frac{\text { av a to }}{\mathrm{H} / \mathrm{U}^{235}}$ & $\frac{r a t i o}{c_{1} \mathrm{U}^{235}}$ & & $\begin{array}{c}\text { dimensions } \\
\text { (in.) }\end{array}$ \\
\hline 0.239 & 13.0 & 106.2 & 32 & $\times 13.5 \times 29.8$ \\
\hline 0.239 & 13.2 & 105.4 & 32 & $\times 12 \times 35.4$ \\
\hline 0.239 & 13.2 & 106.9 & 32 & $\times 9 \times 110$ \\
\hline 0.25 .4 & 12.4 & 99.1 & 64 & $\times 9 \times 39.1$ \\
\hline 0.254 & 12.5 & 99.2 & 64 & $\times 8.25 \times 52$ \\
\hline
\end{tabular}

$\begin{array}{cc}\begin{array}{c}\text { reflector } \\ (1 \text { lucize }) \\ \left({\left.\mathrm{g} / \mathrm{cm}^{3}\right)}^{3}\right.\end{array} & \frac{\mathrm{m}_{\mathrm{c}}}{\left(\mathrm{kg} \mathrm{u}^{235}\right)} \\ 1.001 & 50.4 \\ 1.032 & 53.3 \\ 1.029 & 124.0 \\ 1.042 & 93.8 \\ 1.033 & 115.9\end{array}$


TABLE IIBI

DIPPUSE U(93.1) REPLECTED BY THICK $\mathrm{O}_{2} \mathrm{O}$ OR BO (CAVITY ASSEYBLLBS)

$u^{235}$ enrichment of all uranium $1893.25 \mathrm{w} / 0$; detalled degeriptione is Rof. (60)

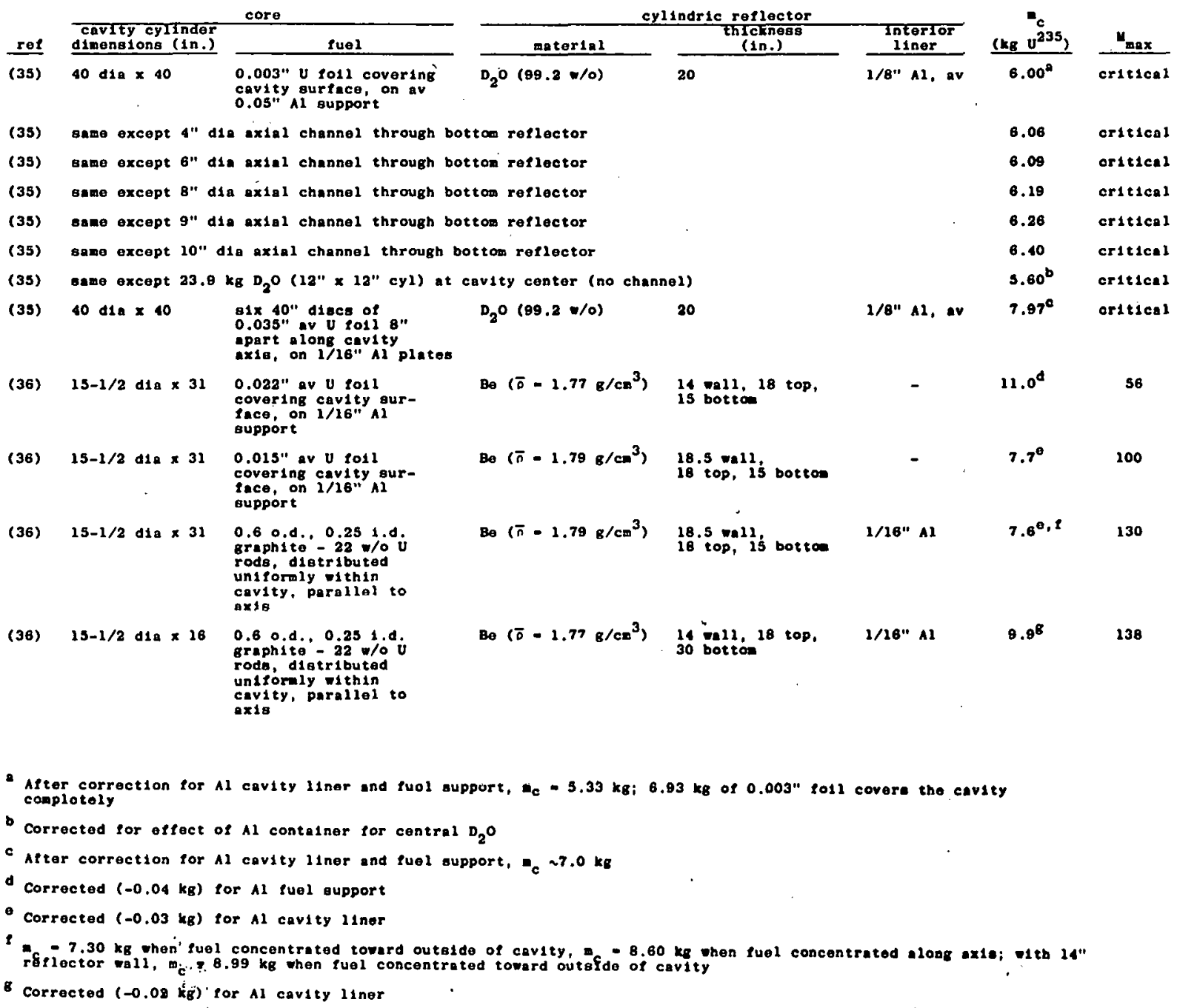


TABLE IIE2

$U(93.65) O_{2} F_{2}-D_{2} O$ SOLUTIONS, BARE, $D_{2} O$ OR GRAPHITE REFLECTED

All systems critica:-

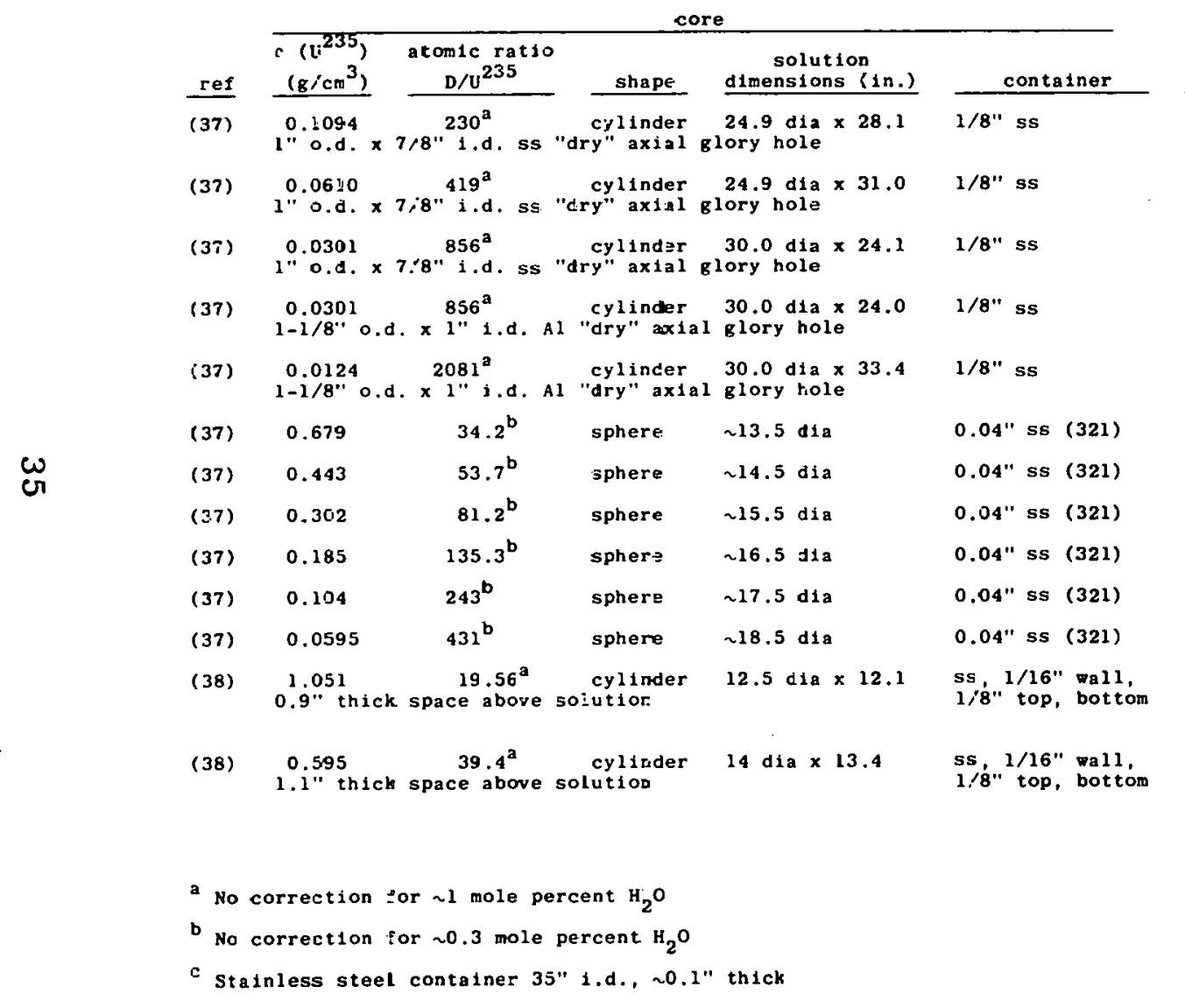

\begin{tabular}{|c|c|c|c|}
\hline \multicolumn{3}{|c|}{ reflector } & \multirow{2}{*}{$\left(\begin{array}{c}\mathrm{mg}_{\mathrm{c}} \\
\mathrm{k}\end{array}\right.$} \\
\hline shape & $\begin{array}{c}\text { anensions } \\
\text { in.) } \\
\end{array}$ & composition & \\
\hline & none & & 24.5 \\
\hline & none & & 15.04 \\
\hline & none & & 8.37 \\
\hline & none & & 8.33 \\
\hline & none & & 4.78 \\
\hline sphere ${ }^{c}$ & 10.7 thick $\mathrm{D}_{2} \mathrm{O}$ & $\sim 99.5 \% \mathrm{D}_{2} \mathrm{O}$ & 14.19 \\
\hline sphere $^{c}$ & 10.2 tiatck $\mathrm{D}_{2} \mathrm{O}$ & $\sim 99.5 \% \mathrm{D}_{2} \mathrm{O}$ & 11.56 \\
\hline sphere ${ }^{c}$ & 9.7 thlek $\mathrm{D}_{2} \mathrm{O}$ & $\sim 99.5 \% \mathrm{D}_{2} \mathrm{O}$ & 9.57 \\
\hline sphere ${ }^{c}$ & 9.2 thick $\mathrm{D}_{2} \mathrm{O}$ & $\sim 99.5 \% \mathrm{D}_{2} \mathrm{O}$ & 7.05 \\
\hline sphere ${ }^{c}$ & 8.7 thick $\mathrm{D}_{2} \mathrm{O}$ & $\sim 99.5 \% \mathrm{D}_{2} \mathrm{O}$ & 4.77 \\
\hline sphere ${ }^{c}$ & 8.2 thick $\mathrm{D}_{2} \mathrm{O}$ & $\sim 99.5 \% \mathrm{D}_{2} \mathrm{O}$ & 3.20 \\
\hline cyl1nder & $\begin{array}{l}32 \text { dis. } x 31.75 \\
9.67 \mathrm{rall}, 10 \text { base, } \\
8.5 \text { tap }\end{array}$ & $\begin{array}{l}\text { graph1te }(\mathrm{Cs}-312) \\
0=1.67 \mathrm{~B} / \mathrm{cm}^{3}\end{array}$ & 25.51 \\
\hline cylinder & $\begin{array}{l}32 \mathrm{did} \times 31.75 \\
8.92 \text { tal1, io base, } \\
7 \text { top. }\end{array}$ & $\begin{array}{l}\text { graph1te }(\mathrm{Cs}-312) \\
\mathrm{c}=1.67 \mathrm{~B} / \mathrm{cm}^{3}\end{array}$ & 20.11 \\
\hline
\end{tabular}

\footnotetext{
No correction for $\sim 0.3$ mole percent $\mathrm{H}_{2} \mathrm{O}$
} 
TABLE IIFIa

$\mathrm{U}(14.67) \mathrm{O}_{2} \mathrm{SO}_{4}-\mathrm{H}_{2} \mathrm{O}$ SOLUTION, SPHERE

Solution volume 14.95 liters, container 12" dia, 1/32" thick, type 347 ss sphere

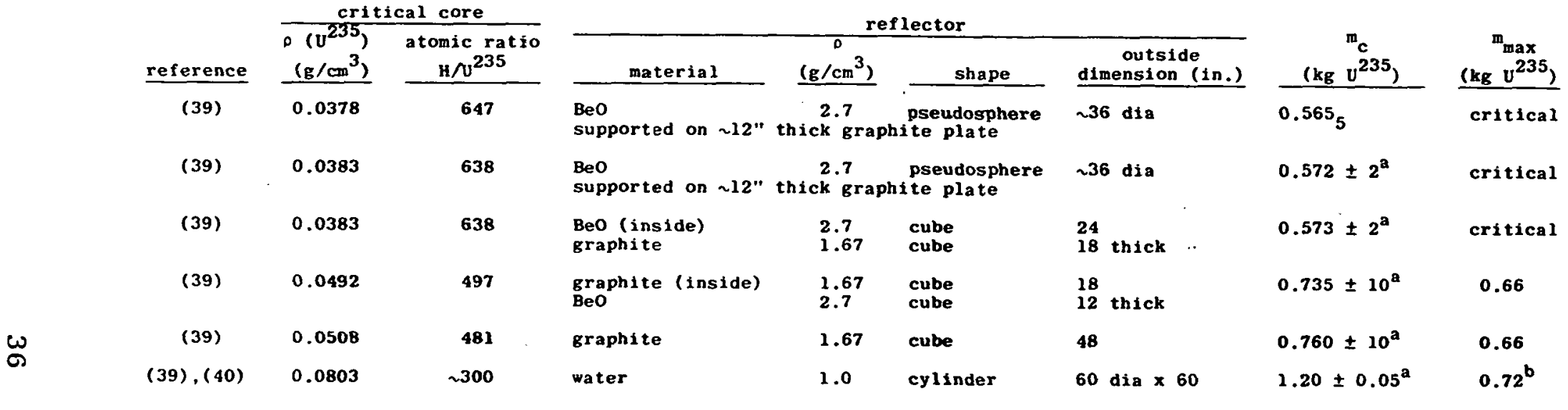

a Large detector displacing reflector near core, re-entrant tube in core Although this measurement does not satisfy the criterion $m_{\max } / m_{c} \geq 0.75$, multiplication curves with several detector types
and locations lead to greater reliability than usual 


\section{TABLE IIFIb}

U(93.5) -PHOSPHAT 3 AQUEOUS SOLUTION, CYLINDERS, 3.0" THICK Fe REFLECTOR

Solutions of $\mathrm{UO}_{3}$ dissolved in 4.26 molar $\mathrm{H}_{3} \mathrm{PO}_{4}$

Solution cylinder $12.4^{\prime \prime} \mathrm{d} i \mathrm{a} ; 1 / 8^{\prime \prime}$ ss (347) container included in thickness of mild steel reflector

\begin{tabular}{|c|c|c|c|c|c|c|c|}
\hline reference & $\begin{array}{c}\text {. }\left(U^{235}\right) \text { of } \\
\text { solution }\left(\mathrm{g} / \mathrm{cm}^{3}\right) \\
\end{array}$ & $\begin{array}{c}\text { atomic ratio } \\
\mathrm{H} / \mathrm{U}^{235} \\
\end{array}$ & $\begin{array}{l}\bar{\rho}(347 \mathrm{ss}) \text { in } \\
\text { core }\left(\mathrm{g} / \mathrm{cm}^{3}\right)^{b} \\
\end{array}$ & $\begin{array}{l}\text { solution } \\
\mathrm{h}_{\mathrm{c}}(\mathrm{in} .) \\
\end{array}$ & $\begin{array}{l}\text { core } \\
\mathrm{h}_{\mathrm{c}} / \mathrm{d}\end{array}$ & $\begin{array}{c}\mathrm{m}_{\mathrm{c}} \\
\left(\mathrm{kg} \mathrm{U}^{235}\right)\end{array}$ & $\begin{array}{r}m_{\max } \\
\left(k g v^{235}\right) \\
\end{array}$ \\
\hline$(41),(42)$ & 0.112 & 212 & 0 & 6.5 & $C .52$ & $1.43 \pm 0.02$ & 1.35 \\
\hline$(42)$ & 0.112 & 212 & 0.725 & 10.0 & $C . .81$ & $2.02 \pm 0.02$ & 1.93 \\
\hline$(42)$ & 0.112 & 212 & 1.140 & 17.1 & 1.38 & $3.26 \pm 0.03$ & 3.10 \\
\hline$(41),(42)$ & 0.101 & 235 & 0 & 6.8 & 0.55 & $1.36 \pm 0.02$ & 1.27 \\
\hline$(42)$ & 0.101 & 235 & 0.725 & 10.8 & 0.87 & $1.97 \pm 0.02$ & 1.85 \\
\hline$(41),(42)$ & 0.090 & 265 & 0 & 7.1 & ").57 & $1.25 \pm 0.02$ & 1.23 \\
\hline (42) & 0.090 & 265 & 0.725 & 12.1 & 0.97 & $1.95 \pm 0.04$ & 1.85 \\
\hline$(41),(42)$ & 0.075 & 321 & 0 & 7.7 & 0.62 & $1.14 \pm 0.02$ & 1.10 \\
\hline (42) & 0.075 & 321 & 0.725 & 15.6 & 1.26 & $2.10 \pm \begin{array}{l}0.12 \\
0.03\end{array}$ & 1.83 \\
\hline
\end{tabular}

a Reflector nearly in contact with top of solution

b Plates of 1/16" thick type 347 stainless steel distributed throughout the solution as vertical grids 
TABLE IIF2

ENRICHED-URANIUU GYDRIDE COMPOSITION"

Cores are homogeneous except that of last entry

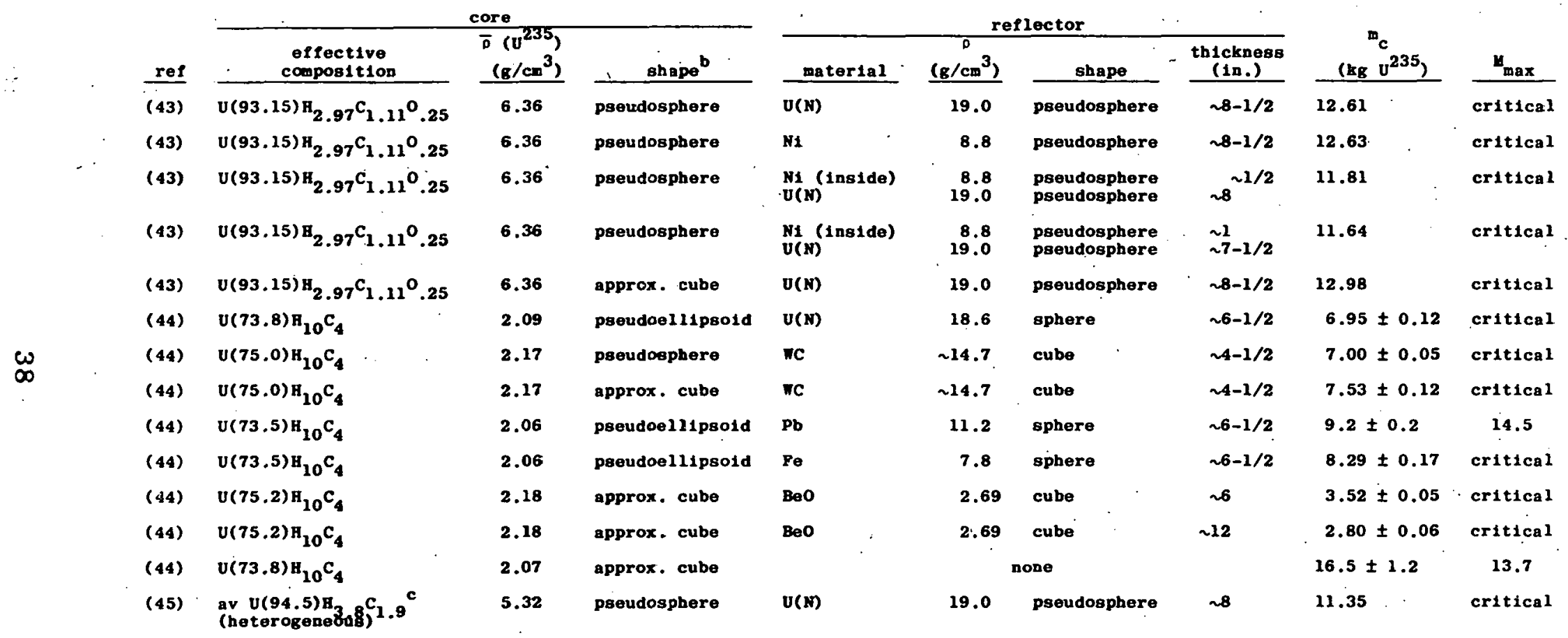

a Heterogeneous nixtures of $\mathrm{U}(72) \mathrm{H}_{10} C_{4}$ and polyethylene extending to the average composition UB80 39 have not been tabulated becaugenens nutures or

b All cores bullt of $1 / 2^{\prime \prime}$ cubic units

c core composed of $1 / 2$ " cubes of $0(94.5$ ) metal and of polgethylene, Intermixed to average $30 \mathrm{v} / 0 \mathrm{U}$ 
TABLE IIF3

LATTICES OF U(94) YETAL UNITS, $\mathrm{H}_{2} \mathrm{O}$ YODERATED, $\mathrm{H}_{2} \mathrm{O}$ REFLECTF"

Contered in water cylinder, 35-1/2" d1a $\times 23^{\prime \prime}$ deep

Relerence (46)

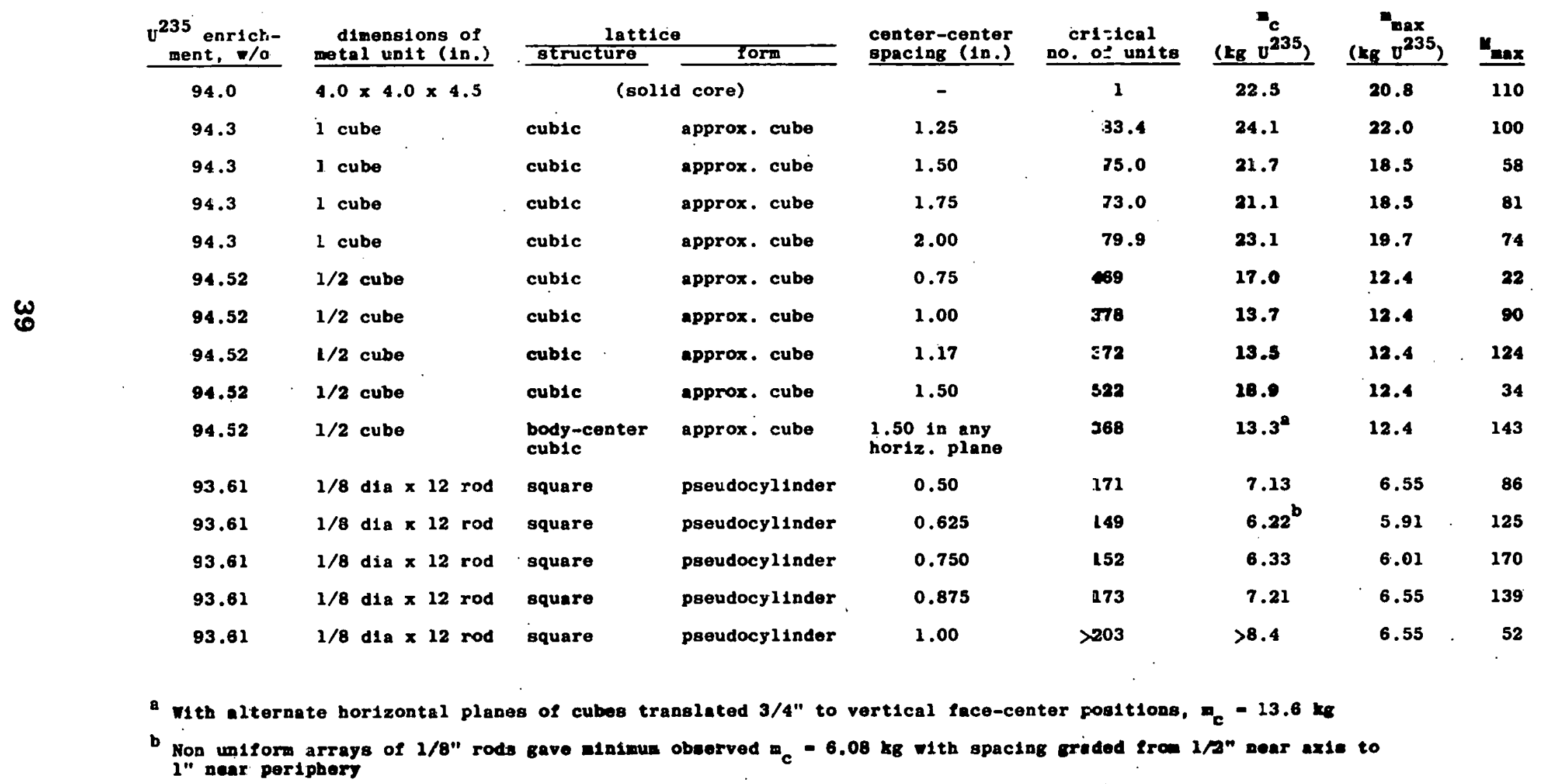




\section{TABLB ITIAI}

\section{PLUTONIUI-MBTAL SPHBRES}

Hemispheres of $\mathrm{Pu}(1.0 \mathrm{~W} / \mathrm{OGa})$ are conted -1 th $0.005^{\prime \prime}$ thick $\mathrm{N}$, unless otherwise noted

\begin{tabular}{|c|c|c|c|c|c|c|c|c|}
\hline \multirow[b]{2}{*}{ rep } & \multicolumn{2}{|c|}{ core } & \multicolumn{4}{|c|}{ reflector } & \multirow[b]{2}{*}{$\begin{array}{c}m_{c} \\
\left(\mathrm{~kg}^{\mathrm{P} v}\right) \\
\end{array}$} & \multirow[b]{2}{*}{$y_{\max }$} \\
\hline & $-1 / 0 \mathrm{pu}^{240}$ & $\begin{array}{l}\overline{\bar{p}}(\text { total Pu }) \\
\left(\mathrm{s} / \mathrm{cm}^{3}\right) \\
\end{array}$ & material & shape & $\begin{array}{l}\text { th1ckness } \\
( \pm n .)\end{array}$ & $\begin{array}{c}\bar{D} \\
\left(\mathrm{~g} / \mathrm{cm}^{3}\right) \\
\end{array}$ & & \\
\hline$(47)(55)$ & 4.5 & 15.45 & & none & & & $16.85 \pm 0.10^{\mathrm{a}}$ & critical \\
\hline (55) & 20.2 & 25.57 & & none & & & $19.26 \pm 0.15^{b}$ & critical \\
\hline (47) (55) & 1.5 & 15.63 & $v(N)$ & pseud os phere & $9-1 / 2$ av & 19.0 & $5.73 \pm 0.02^{c}$ & critical \\
\hline (7) (55) & 4.82 & 15.36 & $U(N)$ & sphero & 7.72 & 19.0 & $5.99 \pm 0.03^{c}$ & critical \\
\hline (2) & 1.35 & 15.58 & $v(N)$ & sphers & 4.60 & 19.0 & 6.22 & 94 \\
\hline (48). & 4.9 & 25.62 & $v(N)$ & sphero & $1.625 \pm 18$ & 18.92 & 8.39 & $m_{\max }>m_{c} e$ \\
\hline (49) (57) & 5.1 & 15.25 & Th & $\begin{array}{l}\text { cylinde } r \\
\text { (21"am } x \text { 21") }\end{array}$ & $8.4 \mathrm{~m} 1 \mathrm{n}$ & 11.58 & $9.24^{a}$ & critical \\
\hline (48) & 4.9 & 15.62 & $n^{p}$ & spher' & $1.850 \pm 18$ & 17.21 & 8.39 & $x>$ \\
\hline (so) & 1.35 & 15.58 & cu & sphers & 5.00 & 8.88 & $6.88^{8}$ & 25 \\
\hline (19) & 4.9 & 15.74 & Al (2014) & sphere & $3.12 \pm 0.03$ & 2.82 & 11.04 & $m_{m g x}>m_{c} e$ \\
\hline (48) & 4.9 & 15.62 & Be $(98-/ 0)$ & sphere & $1.452 \pm 1 \%$ & 2.83 & 8.39 & $x>\infty_{c}^{e}$ \\
\hline (25) & 1.35 & 15.58 & water & cylinder & $>12$ & 1.00 & $7.9^{b}$ & 15 \\
\hline (55) & 5.20 & 19.74 & vater & cylinder & $>10$ & 1.00 & $5.79 \pm 0.3^{1}$ & critical \\
\hline (10) & 1.0 & 15.6 & $\begin{array}{l}\text { Inside: } U(N) \\
\text { outside: } A 1\end{array}$ & $\begin{array}{l}\text { sphera } \\
\text { sphere }\end{array}$ & 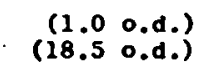 & $\begin{array}{r}19.0 \\
2.7\end{array}$ & $6.46^{\mathrm{h}}$ & $u_{\max }-6.15$ \\
\hline (10) & 1.35 & 15.58 & $\begin{array}{l}\text { Inside: } U(N) \\
\text { outside: } \mathbb{P C}\end{array}$ & $\begin{array}{l}\text { sphere } \\
\text { paralielepipe } \\
\left(12.75^{\prime \prime} \times 12.75^{\prime \prime}\right. \\
\text { x 10.62") }\end{array}$ & od 0.45 & $\begin{array}{r}19.0 \\
\sim 14.7\end{array}$ & $6.13^{h}$ & critical \\
\hline
\end{tabular}

aThree major parts; corrected empirically to unreflected, uniform s:phere; $0.3 \nabla / 0 \mathrm{Pu}^{241}$.

bThree wajor parts; corrected empirically to unreflected, uniform sphere; $3.1 \% / 0 \mathrm{Pu}^{241}, 0.4 \nabla / 0 \mathrm{Pu} 242$.

cTwo major parts, corrected emplrically for effects of $\mathrm{N1}$ and cavities .

$d_{0.30} \approx / 0 \mathrm{Pu}^{241}$.

offect of a small compensating gap was extrapolated to zero.

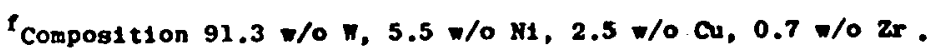

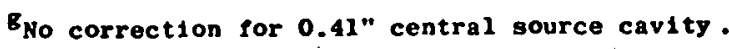

ho correction for 0.83 " central source cavity.

$10.30=/ 0 \mathrm{Pu}^{241}, 0.02 \mathrm{w} / 0 \mathrm{Pu}^{242}$; no Ga. 


\section{TABLE IIIA2}

PLUTONIUM IN METAL-POLYETHYLENE REFLECTOR, SPHERICAL GEOMETRY

Ball of $\mathrm{Pu}\left(94.54 \mathrm{w} / \mathrm{O} \mathrm{Pu}^{239}, 5.11 \mathrm{w} / 0 \mathrm{Pu}^{240}, 0.35 \mathrm{w} / \mathrm{O} \mathrm{Pu}^{241}\right)$ in three major Ni-coated parts, $0.95 \mathrm{w} / \mathrm{O} \mathrm{Ga}$

close-fitting metal or graphite shell, 5.335-cm nominal i.d., $6.335-\mathrm{cm}$ o.d., polyethylene shell $6.342-\mathrm{cm} 1 . \mathrm{d}$.

Critical mass $9.76 \mathrm{~kg}$ pu in each case

Reference (61)

metal or graphite shell material mass (kg)

heryll1um

0.783

graphite

a luminum

Iron

molybdenum

0.738

1.140

3.414

4.291

polye thylene critical parameters ${ }^{a}$ density $\left(\mathrm{g} / \mathrm{cm}^{3}\right) \quad$ mass $(\mathrm{kg})$

$$
0.926 \text { av }
$$

1.261

$\frac{u_{\max }}{549}$
critical
critical
critical
274




TABLE IIIA3

PLUTONIUM-METAL CYLIKDERS

Cores of $\mathrm{Pu}(1 \mathrm{w} / 0 \mathrm{Ga})$ containing $\sim 5 \% \mathrm{Pu}^{240}$; no correction for $\mathrm{NI}$ coating

Reference (12)

See also the last item of Table vB

\begin{tabular}{|c|c|c|c|c|c|c|c|}
\hline \multicolumn{3}{|c|}{ core } & \multicolumn{3}{|c|}{ cylindric reflector } & \multirow[b]{2}{*}{$\left.{ }_{(\mathbf{k g}}^{m_{c}} \mathrm{Pu}\right)$} & \multirow[b]{2}{*}{$\begin{array}{c}m_{\max } \\
\left(\mathbf{k g}_{\mathrm{g}} \mathrm{Pu}\right) \\
\end{array}$} \\
\hline $\begin{array}{r}\text { cylinder } \\
\text { dia (in.) } \\
\end{array}$ & $h_{c} / d$ & $\begin{array}{c}\bar{\rho}(\text { total } P u) \\
\left(\mathrm{g} / \mathrm{cm}^{3}\right) \\
\end{array}$ & material & $\begin{array}{c}\bar{\rho} \\
\left(\mathrm{g} / \mathrm{cm}^{3}\right) \\
\end{array}$ & $\begin{array}{l}\text { thickness } \\
\text { (in.) } \\
\end{array}$ & & \\
\hline $2.25^{a}$ & 8.75 & 15.44 & $\mathrm{u}(\sim 0.3)^{\mathrm{b}}$ & 18.7 & 3.0 & $20.0 \pm 0.1$ & 19.2 \\
\hline $2.25^{a}$ & 7.13 & 15.44 & graphite & 1.60 & 7.0 & $16.3 \pm 0.1$ & 15.7 \\
\hline $2.21^{\mathrm{a}}$ & 12.52 & 15.44 & water & 1.00 & $>12$ & $27.1 \pm 1.5$ & 21.3 \\
\hline $6.0^{c}$ & 0.258 & 14.3 & $U(N)$ & 18.7 & 3.0 & $10.14 \pm 0.07$ & 9.9 \\
\hline $6.0^{c}$ & 0.390 & 14.3 & graphite & 1.60 & 1.0 & $15.44 \pm 0.07$ & 15.4 \\
\hline $6.0^{c}$ & 0.273 & 14.3 & graphite & 1.60 & 7.0 & $10.8 \pm 0.07$ & 10.7 \\
\hline $6.0^{c}$ & 0.280 & 14.3 & water & 1.00 & $>12$ & $11.1 \pm 0.2$ & 9.9 \\
\hline $11.0^{c, e}$ & 0.095 & $13.1^{e}$ & water $d$ & 1.00 & $>12$ & $21.4 \pm 0.8$ & 20.0 \\
\hline $16.0^{\mathrm{c}, \mathrm{e}}$ & 0.049 & $13.1^{e}$ & water & 1.00 & $>12$ & $34.1 \pm 1.2$ & 26.5 \\
\hline
\end{tabular}

a Pu pieces 0.5 " to 3.0" thick, each coated with $0.005^{\prime \prime}$ thick Ni

b Reflector wall lined with 0.030 " thick steel

c Pu pieces 5.934" dia $\times 0.123 "$ in thin Ni cans with outside dimensions 5.967" $\times 0.135 "$

d Core sealed in lucite container before immersion in water

e Average diameter and density of cylinders constructed of overlapping layers of closepacked plates 
TABLE IIIBI

DILUTED PU CYLINDERS, 6.0" DIAMETER, UNREFLECTED

$\mathrm{Pu}(1 \mathrm{w} / \mathrm{O} \mathrm{Ga}), 25 \% \mathrm{Pu}^{240}$, ag discs $5.934 "$ dia $\times 0.123 "$, in thin Ni cans of outside dimensions $5.967 " \mathrm{dia} \times 0.135^{\prime \prime}, \cap^{\prime}(\mathrm{Pu})=15.61 \mathrm{~g} / \mathrm{cm}^{3}$.

Diluent plates $5.967 "$ dia $\times 1 / 8$ " or $1 / 4$ "

Reference (51)

\begin{tabular}{|c|c|c|c|c|c|c|c|c|c|c|}
\hline \multirow[b]{2}{*}{ diluent (A) } & \multirow{2}{*}{$\begin{array}{c}\mathrm{vol} \% \\
\mathrm{Pu}\end{array}$} & \multicolumn{2}{|c|}{$\begin{array}{l}\text { repeated layers, } \\
\text { nom thickness (in.) }\end{array}$} & \multicolumn{3}{|c|}{ core-average $\cap\left(\mathrm{g} / \mathrm{cm}^{3}\right)^{2}$} & \multirow[b]{2}{*}{$h_{c}\left(1 n_{.}\right)$} & \multirow{2}{*}{${ }_{c_{c} / d^{a}}$} & \multirow{2}{*}{$\begin{array}{c}\mathrm{m}_{\mathrm{c}} \\
(\mathrm{kg} \mathrm{Pu}) \\
\end{array}$} & \multirow{2}{*}{$\begin{array}{r}\operatorname{m}_{\max } \\
\left(\mathbf{k g}_{8} \mathrm{Pu}\right) \\
\end{array}$} \\
\hline & & Pu & A & $\overline{\bar{p}(P u)}$ & $\overline{\bar{p}}(\mathrm{~A})$ & $\overline{\bar{p}(\mathrm{N1})}$ & & & & \\
\hline none & 91.4 & $1 / 8$ & - & 14.27 & - & 0.65 & 3.23 & 0.54 & $21.4^{\mathrm{b}}$ & 20.2 \\
\hline$U(0.28)$ & 63.0 & $1 . / 4$ & $1 / 8$ & 9.83 & 5.97 & 0.45 & 6.07 & 1.01 & 27.3 & 25.2 \\
\hline steel $^{c}$ & 62.7 & $1 / 4$ & $1 / 8$ & 9.78 & 2.50 & 0.45 & 7.32 & 1.22 & 32.8 & 27.1 \\
\hline Th & 62.7 & $\therefore / 4$ & $1 / 8$ & 9.78 & 3.62 & 0.45 & 7.85 & 1.31 & 35.2 & 27.1 \\
\hline
\end{tabular}

a Based on 6.00" diameter

b Also reported in (51) are reflector saving values for $1 / 2$ " thick discs of polyethylene, Be, graphite, $\mathrm{Hg}, \mathrm{Al}, \mathrm{TI}, \mathrm{Fe}, \mathrm{Co}, \mathrm{Ni}, \mathrm{Cu}, \mathrm{Mo}, \mathrm{W}, \mathrm{Th}, \mathrm{U}(\mathrm{N})$, and $\mathrm{U}(0.28)$, on the top $\rightarrow P$ this Pu stack

C Stainless steel, type 304 


\section{TABLE IIIB2}

DILUTED PU CYLINDERS, 6.0" DIAMETER, 2.0" THICK U( 0.3) REFLECTOR

$\mathrm{Pu}(1 \mathrm{w} / \mathrm{O} \mathrm{Ca}), 25 \% \mathrm{Pu}^{240}$, as discs $5.934 "$ dia $\times 0.123 "$, in thin Ni cans of outside dimensions $5.967 " \mathrm{dia} \times 0.135 " ; n^{\prime}(\mathrm{Pu})=15.61 \mathrm{~g} / \mathrm{cm}^{3}$

Diluent plates $5.97 "$ dia $x 1 / 8$ " or $1 / 4 "$

Steel guide sleeve, 0.030 " thick, within reflector cylinder; $\bar{r}(U)=19.0$

Reference (51)

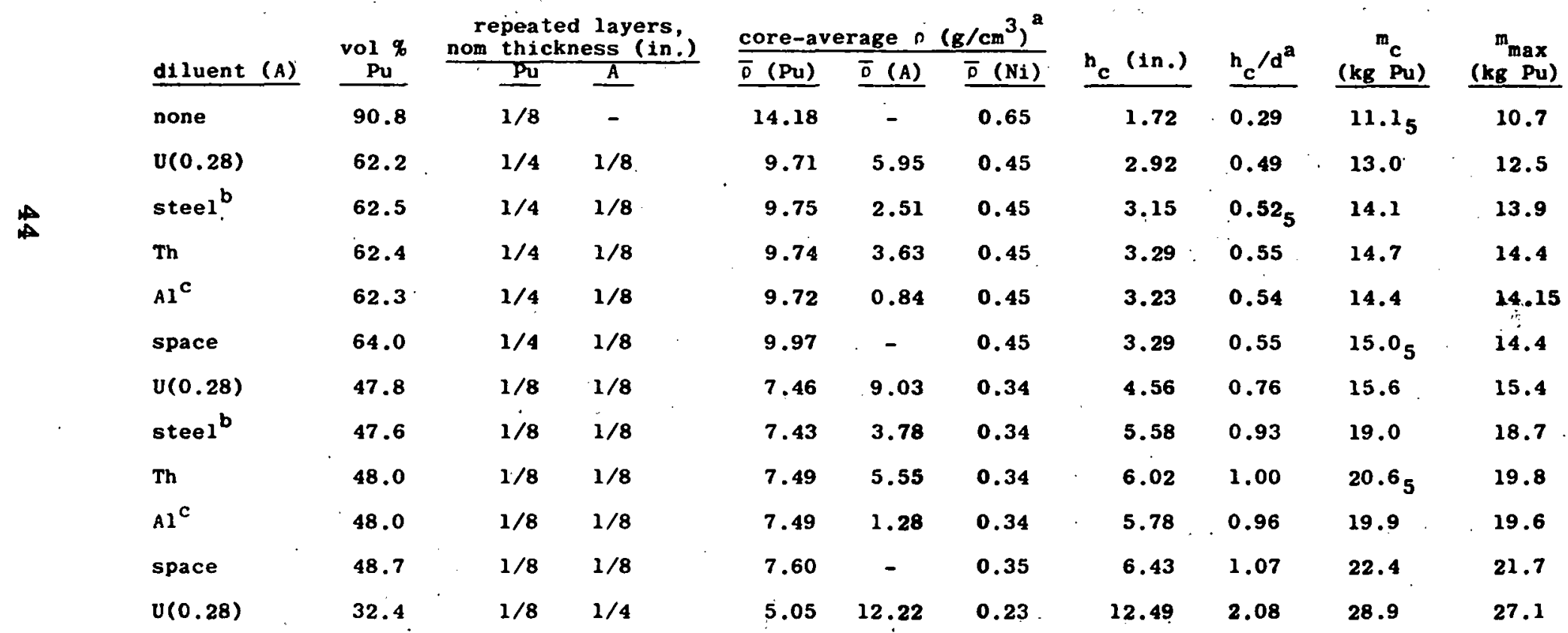

a Based on 6.00" diameter to include reflector clearance

b Stainless steel, type 304

c Aluminum, type $1100 \mathrm{~F}$ 
TABLE IIIB3

DILUTED PU CYLINDERS, 6.0" DIAMET ZR, 4.5" THICK U( 0.3) REFLECTOR

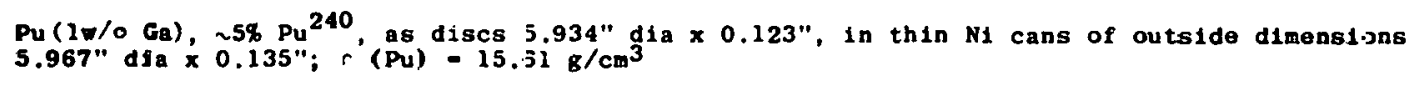

Diluent filates $5.97 "$ "dia $\times 1 / 8$ " or $1 / 4 "$

Steel gulde sleeve, 0.030 " thick, within reflector cyltnder; $\bar{n}(U)=19.0$

Reference (51)

\begin{tabular}{|c|c|c|c|c|c|c|c|c|c|c|c|}
\hline & & vol $\%$ & $\begin{array}{r}\text { repea } \\
\text { nom th1 }\end{array}$ & $\begin{array}{l}\text { layers, } \\
\text { ess (in.) }\end{array}$ & core-ay & rage $D$ & $\left.\mathrm{~g} / \mathrm{cm}^{3}\right)^{a}$ & & & & $a_{\max }$ \\
\hline & diluent (A) & $\mathrm{Pu}$ & Pu & $A$ & $\underline{\bar{\rho}(\mathrm{Pu})}$ & $\bar{D}(A)$ & $\bar{\rho}(\mathrm{N1})$ & $h_{c}(1 n)$. & ${ }_{c} / d^{*}$ & (kg Pu) & $(\mathrm{kg} \mathrm{Pu})$ \\
\hline & none & 91.4 & $1 / 8$ & - & 14.26 & - & 0.65 & 1.42 & 0.24 & 9.3 & 9.0 \\
\hline & $v(0,28)$ & 61.8 & $1 / 4$ & $1 / 8$ & 9.65 & 5.88 & 0.44 & 2.40 & 0.40 & 10.6 & 9.0 \\
\hline & steel ${ }^{b}$ & 60.6 & $1 / 4$ & $1 / 8$ & 9.46 & 2.48 & 0.44 & 2.59 & 0.43 & 11.2 & 10.6 \\
\hline & Th & 62.5 & $1 / 4$ & $1 / 8$ & 9.75 & 3.65 & 0.45 & 2.62 & 0.44 & 11.7 & 10.8 \\
\hline 3 & ${ }_{A 1}{ }^{C}$ & 62.5 & $1 / 4$ & $1 / 8$ & 9.75 & 0.84 & 0.45 & 2.58 & 0.43 & $21.5_{5}$ & 10.8 \\
\hline & space & 63.7 & $1 / 4$ & $1 / 8$ & 9.95 & - & 0.45 & 2.59 & 0.43 & 11.8 & 10.8 \\
\hline & $v(0.28)$ & 47.5 & $1 / 8$ & $1 / 8$ & 7.42 & 9.08 & 0.34 & 3.72 & 0.62 & $12.6_{5}$ & 12.55 \\
\hline & steol ${ }^{b}$ & 47.5 & $1 / 8$ & $1 / 8$ & 7.42 & 3.81 & 0.34 & 4.26 & 0.71 & 14.5 & 14.35 \\
\hline & Th & 47.7 & $1 / 8$ & $1 / 8$ & 7.44 & 5.55 & 0.34 & 4.51 & 0.75 & 15.4 & 15.2 \\
\hline & $\mathrm{Al}^{\mathrm{C}}$ & 47.5 & $1 / 8$ & $1 / 8$ & 7.42 & 1.27 & 0.34 & 4.34 & 0.72 & 14.8 & 14.35 \\
\hline & space & 49.0 & $1 / 8$ & $1 / 8$ & 7.65 & - & 0.35 & 4.46 & 0.74 & 25.7 & 15.4 \\
\hline & $v(0.28)$ & 32.4 & $1 / 6$ & $1 / 4$ & 5.06 & 12.28 & 0.23 & 7.89 & 1.33 & $18.5_{5}$ & 18.0 \\
\hline & steol ${ }^{b}$ & 32.3 & $1 / 8$ & $1 / 4$ & 5.04 & 5.14 & 0.23 & 10.87 & 1.83 & 25.3 & 24.3 \\
\hline & Th & 32.5 & $1 / 8$ & $1 / 4$ & 5.07 & 7.70 & 0.23 & 12.80 & 2.15 & 30.0 & 28.0 \\
\hline & $A 1^{C}$ & 32.4 & 1.8 & $1 / 4$ & 5.05 & 2.75 & 0.23 & 11.42 & 1.90 & 26.4 & 26.1 \\
\hline & space & 32.7 & $1 / 8$ & $1 / 4$ & 5.11 & - & 0.23 & 12.84 & 2.14 & 30.1 & 27.0 \\
\hline
\end{tabular}

a Based on 6.00" dimeter to include reflector clearance

b Stainless steel, type 304

c Alumiaum, tppe 1100F 
TABLE IIIB4

DILUTED PU CYLINDERS, 6.0" DIAMETER, 7.5" THICK U( 0.3) REFLECTOR

$\mathrm{Pu}(1 \mathrm{w} / \mathrm{O} \mathrm{Ga}), 25 \% \mathrm{Pu}^{240}$, as discs 5.934" dia $\times 0.123 "$, in thin Ni cans of outside dimensions

5.967" d1a $\times 0.135^{\prime \prime} ; O^{\prime}(\mathrm{Pu})=15.61 \mathrm{~g} / \mathrm{cm}^{3}$

D1 luent plates $5.97^{\prime \prime}$ d1a $x 1 / 8 "$ or $1 / 4^{\prime \prime}$

Steel guide sleeve 0.030 " thick, within reflector cylinder; $\bar{p}(U)=19.0$

Reference (51)

\begin{tabular}{|c|c|c|c|c|c|c|c|c|c|c|}
\hline \multirow[b]{2}{*}{ diluent (A) } & \multirow{2}{*}{$\begin{array}{c}\text { vol } \% \\
\text { Po }\end{array}$} & \multicolumn{2}{|c|}{$\begin{array}{l}\text { repeated layers, } \\
\text { nom thickness (1n.) }\end{array}$} & \multicolumn{3}{|c|}{ core-average $\circ\left(\mathrm{g} / \mathrm{cm}^{3}\right)^{2}$} & \multirow[b]{2}{*}{$h_{c}\left(1 n_{.}\right)$} & \multirow{2}{*}{$\mathrm{h}_{\mathrm{c}} / \mathrm{d}^{\mathrm{a}}$} & \multirow{2}{*}{$\begin{array}{r}\begin{array}{c}m_{c} \\
\left(k_{g}\right. \\
\left.P_{u}\right)\end{array} \\
\end{array}$} & \multirow{2}{*}{$\begin{array}{r}m_{\max } \\
(\mathbf{k g} \mathbf{P u}) \\
\end{array}$} \\
\hline & & Pu & es (12.) & $\overline{\bar{D}(P u)}$ & $\overline{\bar{\rho}(A)}$ & $\overline{\bar{f}\left(\mathrm{~N}_{1}\right)}$ & & & & \\
\hline none & 91.2 & $1 / 8$ & - & 14.23 & - & 0.65 & 1.37 & 0.23 & $8.9_{5}$ & 8.0 \\
\hline$v(0.28)$ & 62.6 & $1 / 4$ & $1 / 8$ & 9.77 & 5.94 & 0.45 & 2.31 & $0.38_{5}$ & $10.3_{5}$ & 10.3 \\
\hline steed $^{b}$ & 62.5 & $1 / 4$ & $2 / 8$ & 9.76 & 2.51 & 0.45 & 2.43 & $0.40_{5}$ & 10.9 & 10.8 \\
\hline Th & 62.5 & $1 / 4$ & $1 / 8$ & 9.76 & 3.63 & 0.45 & 2.49 & $0.41_{5}$ & 11.1 & 10.8 \\
\hline$A 1^{C}$ & 62.5 & $1 / 4$ & $1 / 8$ & 9.76 & 0.84 & 0.45 & 2.47 & 0.41 & $11.0_{5}$ & 10.8 \\
\hline space & 63.5 & $1 / 4$ & $1 / 8$ & 9.92 & - & 0.45 & 2.47 & 0.41 & $11.2_{5}$ & 10.8 \\
\hline$v(0.28)$ & 47.7 & $1 / 8$ & $3 / 8$ & 7.44 & 9.05 & 0.34 & 3.51 & $0.58_{5}$ & $11.9_{5}$ & 11.7 \\
\hline $\operatorname{steel}^{b}$ & 47.7 & $1 / 8$ & $1 / 8$ & 7.45 & 3.80 & 0.34 & 3.97 & $0.66_{5}$ & $13.5_{5}$ & 13.3 \\
\hline $\mathbf{T h}$ & 48.1 & $1 / 8$ & $1 / 8$ & 7.51 & 5.57 & 0.34 & 4.14 & 0.69 & $14.2_{5}$ & 14.2 \\
\hline$A I^{C}$ & 48.0 & $1 / 8$ & $1 / 8$ & 7.50 & 1.28 & 0.34 & 3.98 & $0.66_{5}$ & 13.7 & 13.5 \\
\hline space & 48.8 & $1 / 8$ & $1 / 8$ & 7.62 & - & 0.35 & 4.18 & 0.70 & 14.6 & 14.4 \\
\hline$v(0.28)$ & 32.4 & $1 / 8$ & $1 / 4$ & 5.06 & 12.27 & 0.23 & 7.29 & 1.22 & 16.9 & 16.7 \\
\hline steel $^{b}$ & 32.1 & $1 / 8$ & $1 / 4$ & 5.01 & 5.11 & 0.23 & 9.49 & 1.58 & 21.8 & 21.7 \\
\hline Th & 32.5 & $1 / 8$ & $1 / 4$ & 5.07 & 7.49 & 0.23 & 10.83 & 1.80 & $25.1_{5}$ & 25.0 \\
\hline${ }_{A 1}{ }^{C}$ & 32.4 & $1 / 8$ & $3 / 4$ & 5.05 & $1.75^{\circ}$ & 0.23 & 9.65 & 1.61 & $22.3_{5}$ & 22.3 \\
\hline space & 32.8 & $1 / 8$ & $1 / 4$ & 5.12 & - & 0.23 & 10.58 & 1.76 & $24.8_{5}$ & 24.4 \\
\hline$v(0.28)$ & 24.4 & $1 / 8$ & $3 / 8$ & 3.81 & 13.87 & 0.17 & 17.24 & 2.87 & 30.1 . & 27.0 \\
\hline
\end{tabular}

a Based on 6.00" diameter to include reflector clearance

b Stainiess steel, type 304

c Aluminum, type 1100F 


\section{TABLE IIIB5}

DILUTED Pu CYLINDERS, 6.0" DIAMETER, 2.0" THICK Th REFLECTOR

$\mathrm{Pu}(1 \mathrm{w} / \mathrm{O} \mathrm{Ga}), 25 \% \mathrm{Pu}^{240}$, as discs $5.934 "$ dia $\times 0.123 "$, In thin Ni cans of outside dimensions $5.967 " \mathrm{dia} \times 0.135^{\prime \prime} ; D^{\prime}(\mathrm{Pu})=15.61 \mathrm{~g} / \mathrm{cm}^{3}$

Diluent plates $5.97^{\prime \prime}$ dia $x 1 / 8 "$ or $1 / 4 "$

Steel guide sleeve, $0.030 "$ thick, within reflector cylinder; $\overline{0}(\mathrm{Th})=11.9 \mathrm{~g} / \mathrm{cm}^{3}$

Reference (51)

\begin{tabular}{|c|c|c|c|c|c|c|c|c|c|c|}
\hline & vol \% & $\begin{array}{c}\text { repea } \\
\text { nom this }\end{array}$ & $\begin{array}{l}\text { ted layers, } \\
\text { akness (in.) }\end{array}$ & core-av & rage $p$ & $\left.\mathrm{~g} / \mathrm{cm}^{3}\right)^{a}$ & & & & $m_{\max }$ \\
\hline diluent $(\mathrm{A})$ & $\mathbf{P u}$ & $\mathrm{Pu}$ & A & $\bar{\rho}(\mathrm{Pu})$ & $\bar{D}(A)$ & $\bar{\rho}(\mathrm{Ni})$ & $h_{c}(1 n)$. & $h_{c} / d^{a}$ & (kg Pu) & $(\mathbf{k g} \mathrm{Pu})$ \\
\hline none & 91.3 & $1 ; 8$ & - & 14.25 & - & 0.65 & 2.25 & $0.37_{5}$ & 14.7 & 14.5 \\
\hline$U(0.28)$ & 63.1 & $1 ; 4$ & $1 / 8$ & 9.85 & 5.29 & 0.45 & 3.90 & 0.65 & 17.6 & 16.3 \\
\hline steel $^{b}$ & 62.8 & $1: 4$ & $1 / 8$ & 9.81 & 2.51 & 0.45 & 4.32 & 0.72 & 19.4 & 18.1 \\
\hline Th & 62.8 & 1,4 & $1 / 8$ & 9.81 & 3.63 & 0.45 & 4.44 & 0.74 & 20.0 & 18.1 \\
\hline$A 1^{C}$ & 62.8 & $1 . / 4$ & $1 / 8$ & 9.81 & 0.84 & 0.45 & 4.46 & 0.74 & $20.0_{5}$ & 18.1 \\
\hline space & 64.0 & $1 / 4$ & $1 / 8$ & 9.99 & - & 0.45 & 4.80 & 0.80 & 22.0 & 19.9 \\
\hline$U(0.28)$ & 47.5 & 1.8 & $1 / 8$ & 7.41 & 9.01 & 0.34 & 6.55 & 1.09 & $22.2_{5}$ & $2 ! .65$ \\
\hline steel $^{b}$ & 50.4 & $1 / 8$ & $1 / 8$ & 7.87 & 4.01 & 0.36 & 8.50 & 1.42 & 30.6 & 26.1 \\
\hline Th & 47.9 & $1 / 8$ & $1 / 8$ & 7.48 & 5.54 & 0.34 & 9.78 & 1.63 & 33.5 & 26.1 \\
\hline$A 1^{C}$ & 48.1 & $1 / 8$ & $1 / 8$ & 7.51 & 1.29 & 0.34 & 10.15 & 1.69 & 34.9 & 36.1 \\
\hline
\end{tabular}

a Based on 6.00" diameter to include reflector clearance

b Stainless steel, type 304

c Aluminum, type $1100 \mathrm{~F}$ 
TABLE IIIB6

DILUTED PU CYLINDFRS, 6.0" DIAMETER, 4.5" THICK Th REFLECTOR

$\mathrm{Pu}(1 \mathrm{w} / 0 \mathrm{Ga}), 25 \% \mathrm{Pu}^{240}$, as discs 5.934" $\mathrm{dia} \times 0.123^{\prime \prime}$, In thin $\mathrm{N} 1$ cans of outside dimensions $5.967 " \mathrm{dia} \times 0.135^{\prime \prime} ; D^{\prime}(\mathrm{Pu})=15.61 \mathrm{~g} / \mathrm{cm}^{3}$

Diluent plates $5.97^{\prime \prime}$ dia $\times 1 / 8$ " or $1 / 4^{\prime \prime}$

Steel guide sleeve, $0.030^{\prime \prime}$ thick, within reflector cylinder; $\bar{p}$ (Th) $-11.9 \mathrm{~g} / \mathrm{cm}^{3}$

Reference (51)

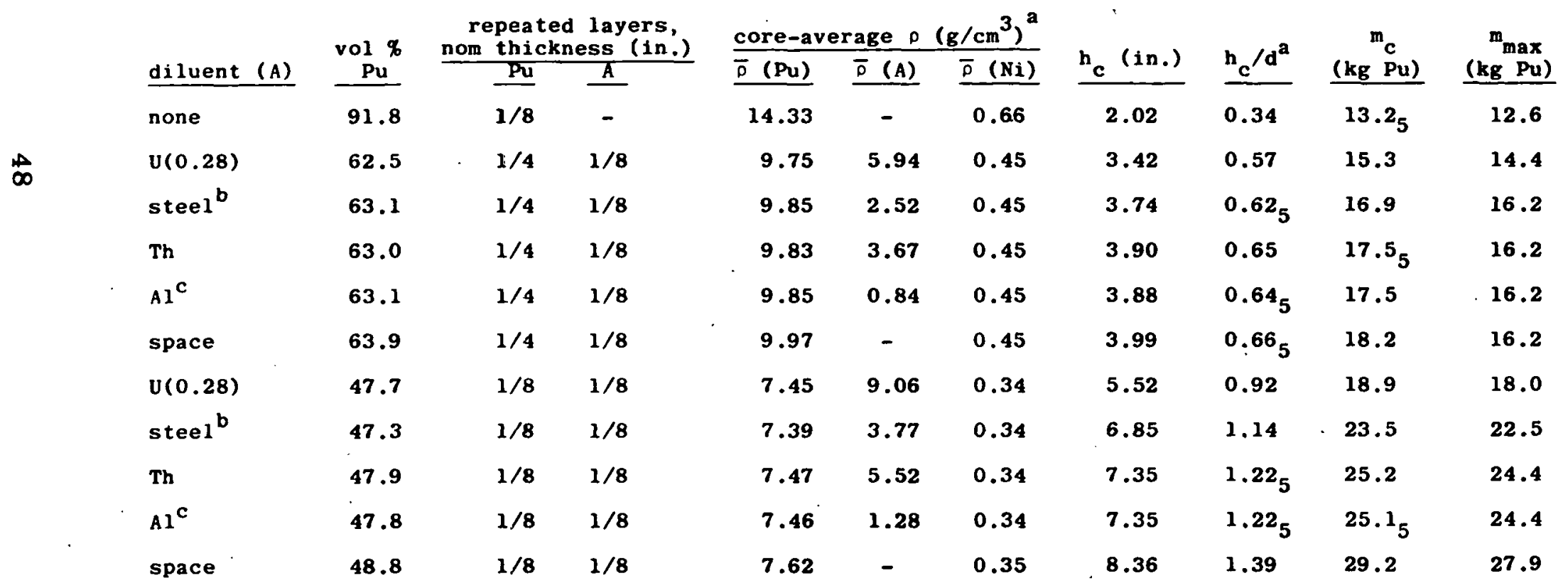

a Based on 6.00" diameter to include reflector clearance

b stainless steel, type 304

c Aluminum, type $1100 \mathrm{~F}$ 
TABLE IIIB7

DILUTED PU CYLINDERS, 6.0" DIAMETER, 7.5" THICK Th REFLECTOR



Diluent plates 5.97 " dia $\times 1 / 3^{\prime \prime}$ or $1 / 4^{\prime \prime}$

Steel guide sleeve, $0.030^{\prime \prime}$ thick, within reflector cylinder; $\overline{0}(\mathrm{Th})-11.9 \mathrm{~g} / \mathrm{cm}^{3}$

Reference (51)

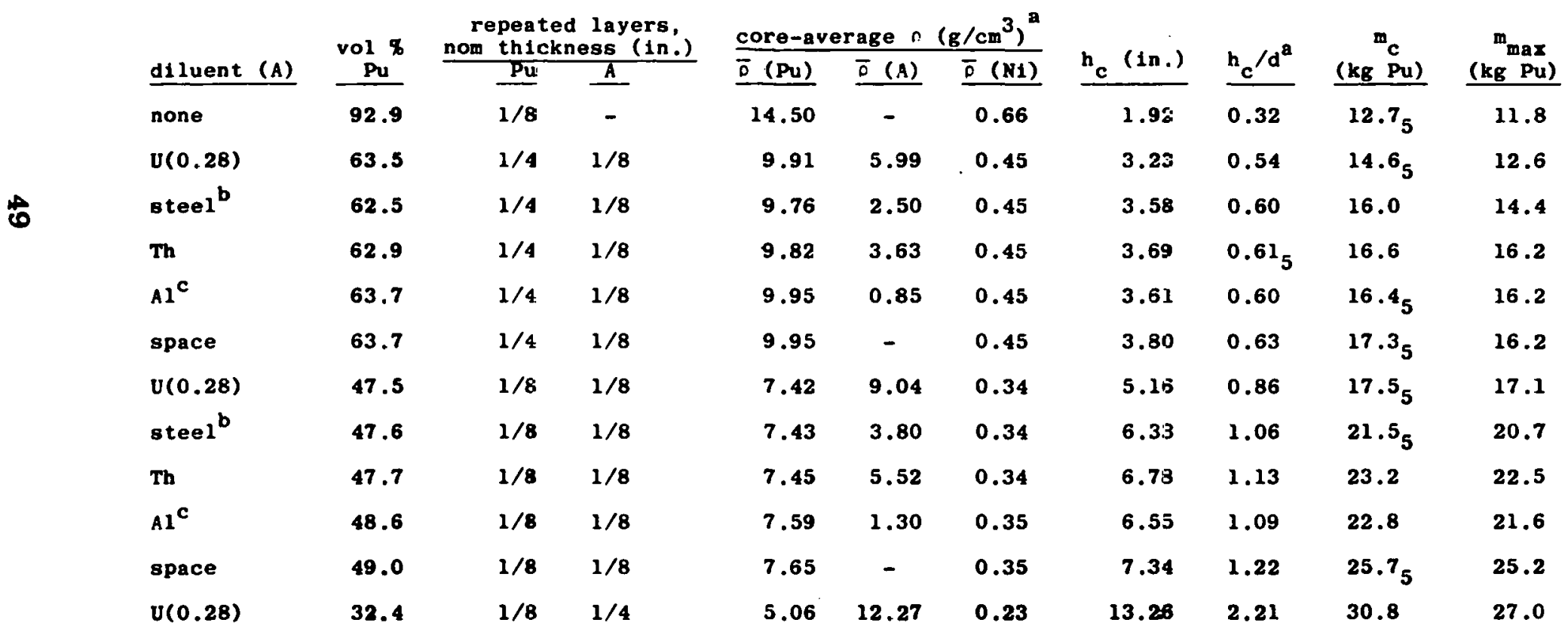

\footnotetext{
a Based on 6.00" diameter to include reflector clearance

b Stalnless steel, type 304

c Aluminum, type $1100 \mathrm{~F}$
} 
TABLE IV

U-233 METAL SPHERES

Reflected cores consist of hemispheres coated with $0.005^{\prime \prime}$ thick N1

\begin{tabular}{|c|c|c|c|}
\hline & co & & \\
\hline 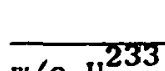 & composition & $v^{238}$ & $\bar{D}(\mathrm{U})$ \\
\hline
\end{tabular}

(1)(55) $98.11 \quad 1.25$

(52) (55) $98.11^{b}$

(48)

98.2

1.25

0.61

28.42

0.61

18.42

0.7

18.64

0.7

18.62

0.7

18.64

0.7

18.62

0.7

18.64

0.7

18.62

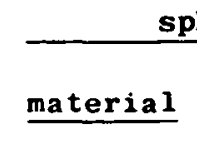

sph

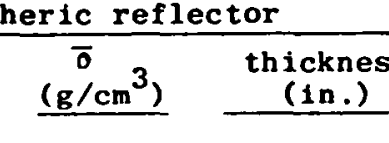

$\frac{\left(\mathrm{kg} \mathrm{v^{233 }}\right)}{16.22 \pm 0.06^{\mathrm{m}}}$

$\frac{m_{\max }}{\text { critical }}$

U(N)

none

19.0

7.84

$5.63 \pm 0.03^{c}$

critical

U(N)

$18.922 .09 \pm 1 \%$

$7.47^{d}$

(3.622" dia)

$>m_{c}{ }^{e}$

$18.920 .906 \pm 1 \%$

$9.84^{d}$

(3.972" dia)

$>m_{c} e$

$w-a 110 y^{f}$

17.21

$2.28 \pm 1 \%$

$7.47^{d}$

(3.622" dia)

W-alloy

17.2

$0.960 \pm 19$

$9.84^{d}$

(3.972" dia)

Be (98\%)

1.83

$1.652 \pm 1 \%$

$7.47^{d}$

(3.622" dia)

He (98\%)

1.83

$0.805 \pm 1 \%$

$9.84^{d}$

(3.972" d1a) $>m_{c}{ }^{e}$

$>m_{c} e$

$>\operatorname{mo}_{c} e$

$>m_{c}{ }^{e}$

a Corrected for effects of $N i$ coating, supports and small asphericity; $0.03 w / 0.0^{235}$

b Analysis available for one hemisphere only, $0.03 \approx / 0 v^{235}$

c corrected for effects of $N i$ coating, oversize core and compensating gap between core and reflector

d Corrected for effects of $v_{i}$ and clearances between assembly parts

e Effect of small compensating gap was adjusted to zero; reflector thickness modified

f Composition $91.3 \mathrm{w} / 0 \mathrm{~W}, 5.5 \mathrm{w} / \mathrm{ON}, 2.5 \mathrm{w} / \mathrm{O} \mathrm{Cu}, 0.7 \mathrm{w} / \mathrm{Or}$ 
TABLE VA

PU OR U-233 METAL SPHERES HITHIN U( ( 93) METAL SPHERES

\begin{tabular}{|c|c|c|c|c|c|}
\hline \multirow[b]{2}{*}{ ref } & \multicolumn{3}{|c|}{ contral ball } & \multicolumn{2}{|c|}{$\mathrm{U}\left(\right.$ (93) sholl $\overline{0}-28.8 \mathrm{~g} / \mathrm{cm}^{3}$} \\
\hline & composition & $\begin{array}{l}\bar{D}\left(\mathrm{Pu}, \mathrm{C}^{(i)}\right) \\
\left(\mathrm{g} / \mathrm{cm}^{3}\right) \\
\end{array}$ & $\begin{array}{r}\text { diain } \\
\text { (iit.) }\end{array}$ & $\begin{array}{l}\text { anricking } \\
\forall / 0 \mathrm{U}^{2}\end{array}$ & $\begin{array}{l}\text { critical } \\
\text { thickness (in.) }\end{array}$ \\
\hline $\begin{array}{l}(48) \\
(55)\end{array}$ & $\begin{array}{l}\text { Pu( }(1 \text { w/o } \bar{g}), \\
4.9 \% \text { Pu240 }\end{array}$ & 25.62 & 3.970 & 93.2 & $0.655 \pm 1 \%$ \\
\hline (53) & $\begin{array}{l}\text { Pu }\left(i: \nabla / z_{4} \dot{b}^{a}\right) \text {. } \\
1.5: P^{2}\end{array}$ & 15.56 & $\begin{array}{l}3.510 \\
0.421 . d .\end{array}$ & 93.18 & 1.006 \\
\hline $\begin{array}{l}(53) \\
(57)\end{array}$ & 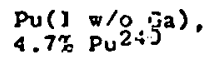 & 25.13 & 2.506 & 93.17 & 1.939 \\
\hline $\begin{array}{l}(53) \\
(57)\end{array}$ & $\begin{array}{l}\text { Pus(: } w /\left(y, \bar{j}^{a}\right) . \\
1.5 \% \text { Pu }\end{array}$ & 25.14 & 2.506 & 93.17 & 1.929 \\
\hline $\begin{array}{l}(53) \\
(57)\end{array}$ & $\begin{array}{l}P_{2}(100 \%) \\
4.81 P_{0}\end{array}$ & 18.80 & 2.502 & 93.17 & 1.644 \\
\hline (s4) & $\begin{array}{l}\text { Pu (100\%) } \\
2.34 \% \text { Pu240 }\end{array}$ & 29.48 & 2.230 & 93.2 & 0.974 \\
\hline (54) & $\begin{array}{l}P_{21}(100 \%) \\
4.73 \% \text { Pri } 240\end{array}$ & 29.42 & 2.130 & 93.2 & 0.988 \\
\hline (54) & $\begin{array}{l}\mathrm{Pu}(100 \%) \\
16.1 \% \mathrm{Pu} 210\end{array}$ & 19.43 & 2.130 & 93.2 & 2.039 \\
\hline $\begin{array}{l}(49) \\
(55)\end{array}$ & $\begin{array}{l}\mathrm{v}^{233} \\
\left(0 s .3 \times \operatorname{sio}^{\circ}\right.\end{array}$ & 28.62 & 3.972 & 93.2 & $0.481 \pm 2 x$ \\
\hline $\begin{array}{l}(19) \\
(55)\end{array}$ & $\begin{array}{l}u^{223} \\
(9 \varepsilon, 11 \otimes, 0)^{0}\end{array}$ & 18.64 & 3.622 & 93.2 & $0.783 \pm 1 \%$ \\
\hline $\begin{array}{l}(53) \\
(57)\end{array}$ & $\begin{array}{l}u^{253} \\
(97.0-/ 0)^{s}\end{array}$ & 17.78 & 2.504 & 93.17 & 1.886 \\
\hline
\end{tabular}

\begin{tabular}{|c|c|c|c|}
\hline \multirow{2}{*}{$\begin{array}{c}U(N) \text { reflector } \\
\text { sphere } \\
\overrightarrow{0}-19.0 \mathrm{~g} / \mathrm{cm}^{3} \\
\text { th1ckness }(1 \mathrm{n} .: \\
\end{array}$} & \multicolumn{2}{|c|}{$m_{c}$} & \multirow[b]{2}{*}{$H_{\max }$} \\
\hline & $\mathrm{kg}$ Pu or $v^{233}$ & $\mathrm{~kg} \mathrm{u^{235 }}$ & \\
\hline none & 8.386 & $12.73 \pm 0.18^{\circledR}$ & $m_{\max }>m_{c}^{b}$ \\
\hline none & 5.72 & $28.8 \pm 0.3^{2}$ & 65 \\
\hline none & $2.02{ }_{2}$ & $36.7 \pm 0.1^{2}$ & 130 \\
\hline none & $2.02_{4}$ & $36.3_{5} \pm 0.1^{2}$ & 118 \\
\hline none & 2.527 & $26.8 \pm 0.1^{c}$ & 233 \\
\hline 7.45 & 1.615 & $8.87^{d}$ & critical \\
\hline 7.43 & 1.610 & $9.09^{d}$ & critical \\
\hline 7.38 & 1.612 & $9.90^{d}$ & $\begin{array}{l}\max _{\max }- \\
0.750^{235}\end{array}$ \\
\hline none & 9.83 & $8.64 \pm 0.11^{*}$ & $m_{\max }>a_{c}^{b}$ \\
\hline none & 7.46 & $13.84 \pm 0.19^{\circ}$ & $m_{\max }>m_{c}^{b}$ \\
\hline none & $2.37 \mathrm{y}$ & $34.8 \pm 0.2^{2}$ & 138 \\
\hline
\end{tabular}

acorrected for Effecte of 0.005 " thuck $N 1$ on $P U$ or $U^{233}$ hemlsphereiz and for clearances between essembly parts

beffect of smal: compensating gap was adjusted to zero; reflector thickness modifled

cCorrocted for offects of $0.005 "$ trlck cu about Pu sphere and for alearances between aesembly parts

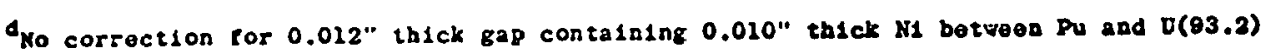

$0_{1.25} \nabla / 0 u^{234}, 0.03 \approx / \circ u^{235}, 0.61 \approx / 0 u^{238}$

$t_{0.0}=/ 0 \mathrm{~J}^{234}, 0.2 \nabla / 0 \mathrm{v}^{238}, 0.95 \nabla / 0 \nabla$ 
TABLE VB

PU METAL CYLINDER WITHIN U(93.2) METAL CYLINDER, THICK U(N) REFLECTOR

The $\mathrm{Pu}(1 \mathrm{w} / \mathrm{O} \mathrm{Ga})$ contains $26 \% \mathrm{Pu}^{240}$; Pu pieces coated with 0.005 " thick $N$

Dimensions of $\mathrm{Pu}$ and outside dimensions of $\mathrm{U}(93.2)$ are such that $h / d$ values are the same

Cores are approximately centered in a $U(N)$ cylinder, $18.0^{\prime \prime}$ dia $\times 10^{\prime \prime}$, of density $19.0 \mathrm{~g} / \mathrm{cm}^{3}$

Reference (53)

\begin{tabular}{|c|c|c|c|c|c|c|c|}
\hline \multirow{2}{*}{\multicolumn{2}{|c|}{$\begin{array}{c}h_{c} / d \\
\left(P u \text { and } v^{235}\right) \\
\end{array}$}} & \multirow{2}{*}{$\begin{array}{c}\text { Pu cavity } \\
\text { dimensions (in.) }\end{array}$} & \multirow{2}{*}{$\begin{array}{r}\bar{D}(\mathrm{Pu}) \\
\left(\mathrm{g} / \mathrm{cm}^{3}\right) \\
\end{array}$} & \multirow{2}{*}{$\begin{array}{l}\bar{p}(U-93.2) \\
\left(\mathrm{g} / \mathrm{cm}^{3}\right) \\
\end{array}$} & \multicolumn{2}{|c|}{$m_{c}{ }^{a}$} & \multirow[b]{2}{*}{$\mathrm{m}_{\max }$} \\
\hline & & & & & kg Pu & $\mathrm{kg} \mathrm{U^{235 }}$ & \\
\hline & 0.20 & 4.315 dia $\times 0.875$ & 14.98 & 18.66 & 3.14 (f 1 xed) & $13.0 \pm 0.2$ & $11.9 \mathrm{~kg} \mathrm{v}^{235}$ \\
\hline & 0.30 & $4.315 \mathrm{dia} \times 1.290$ & 15.29 & 18.30 & 4.73 (fixed) & $5.3 \pm 0.2$ & $4.2 \mathrm{~kg} \mathrm{v}^{235}$ \\
\hline & 1.00 & 2.235 dia $\times 2.231$ & 14.83 & 18.58 & 2.13 (fixed) & $9.7 \pm 0.2$ & $8.2 \mathrm{~kg} \mathrm{v}^{235}$ \\
\hline & 0.44 & $4.315 \mathrm{dia} c y l$ & 15.34 & none & $6.91 \pm 0.04$ & - & $6.47 \mathrm{~kg} \mathrm{Pu}$ \\
\hline
\end{tabular}

a No correction for effect of $\mathrm{Ni}$ or 0.06 in. ${ }^{3}$ central source cavity 


\section{REF ERENCES}

(1) G. E. Hansen, Status of Computational and Experimental Correlations for Los Alamos Fast-Neutron Critical Assembiles, Physics of Fast and Intermediate Reactors, TAEA, Vieñna (1962).

(2) G. E. Hansen and D. P. Wood, Precision Critical-Mass Determinations for Oralloy and Plutonium in spherical TubaIloy Tampers, LA-1356 Revised (to be issued).

(3) J. D. Orndoff and H. C. Paxton, Measurements on Untamped Oralloy Assembly, LA-1209 (February 1951).

(4) V. Josephson, R. W. Paine, Jr. and L. L. Woodward, Oralloy Shape Factor Measurements, LA-1155 (August 1950).

(5) G. E. Hansen, D. P. Wood and B. Peña, Reflector Savings of Moderating Materials on Large Diameter U(93.2\%) STabs, LAMS-2744 (June 1962).

(6) H. C. Paxton, Bare Critical Assemblies of Oralloy at Intermediate Concentrations of U-235, LA-1671 May 1954).

(7) Private communication, D. M. Barton (September 1958).

(8) V. Josephson, Critical Mass Measurements on Oy in Tu and WC Tampers, LA-III4 deleted (May 1950).

(9) J. D. Orndoff, H. C. Paxton and G. E. Hansen, Critical Masses of Oralloy at Reduced Concentrations and Densities, LA-1251 (May 195I).

(10) Private communication, C. P. Baker (December 1947).

(11) G. E. Hansen, H. C. Paxton and D. P. Wood, Critical Masses of Oralloy in Thin Reflectors, LA-2203 (July 1958). 


\section{REF ERENCES (Continued)}

(12) G. E. Hansen, H. C. Paxton and D. P. Wood, Critical Plutonium and Enriched-Uranium-Metal Cylinders of Extreme Shape, Nuclear Sci. and Eng . 8, 570-577 (1960).

(13) J. J. Neuer, Critical Assembly of Uranium Metal at an Average U235 Concentration of 16-1/4\%, LA-2085 (January 1957).

(14) Private communication, J. J. Neuer, G. A. Newby, H. C. Paxton and T. F. Wimett (March 1954).

(15) Private communication, H. C. Paxton and C. B. Stewart (May 1953).

(16) Private communication, D. P. Wood, L. C. Osborn and B. Peña (April 1960).

(17) Private communication, V. Josephson and R. W. Paine, Jr. (March 1951).

(18) Private communication, J. C. Hoogterp and D. P. Wood (September 1955).

(19) D. P. Wood and B. Peña, Critical Mass Measurements of Oy and $\mathrm{Pu}$ Cores in Spherical Aluminum Reflectors, LAMS-2579 (June 1961).

(20) E. C. Mallary, Oralloy Cylindrical Shape Factor and Critical Mass Measurements in Graphite, Paraffin, and Water Tampers, LA-1305 (October 1951).

(21) J. C. Hoogterp, Critical Masses of Graphite-Tamped Heterogeneous Oy-Graphite Systems, LA-1732 (May I954).

(22) Private communication, G. A. Jarvis (November 1961).

(23) Private communication, D. P. Wood (October 1961).

(24) Private communication, F. F. Hart and C. B. Stewart (December 1953).

(25) Private communication, R. W. Paine, Jr., D. P. Wood and R. S. Dike (April 1951). 


\section{REFERENCES（Continued）}

(26) F. F. Hart, Safety Tests for Melting and Casting Ora11oy, LA-1623 (December 1953).

(27) Private communication, K. Gallup, G. E. Hansen (July 1951).

(28) Private communication, R. E. Schreiber (September 1061).

(29) Private communication, E. C. Mallary (March 1952).

(30) Private communication, J.J. Neuer, H. C. Paxton, R. H. White and T. F. Wimett (March 1954).

(31) Private communication, G. A. Jarvis (June 1963).

(32) H. Iskenderian and C. C. Byers, Physics Calculations on Four Los Alamos Graphite Moderated Critical Assemblies, Trans. ANS 1 , No. 1, p. 149 (June 1958); also private communication, C. C. Byers (September 1957).

(33) G. E. Hansen, J. C. Hoogterp, J. D. Orndoff and H. C. Paxton, Beryllium-Reflected, Graphite-Moderated Critical Assembiles, LA-2141 (October 1957).

(34) Private communication, S. J. Balestrini (November 1963).

(35) Private communication, C. C. Byers (March 1962).

(36) Private communication, G. A. Jarvis and C. C. Byers (October 1961).

(37) R. N. Olcott, Homogeneous Heavy Water Moderated Critical Assemblies, Part 1, Experimenta1, Nuclear Sci. and Eng. I, 327-341 (1956).

(38) G. E. Hansen and W. H. Roach, Interpretation of Neutron Resonance Detector Activities in Critical Uranyl Fluoride - Heavy Water Solutions, Proc. Brookhaven Conf. on Resonance Absorption of Neutrons in Nuclear Reactors, Upton, New York, BNL 433 (C-24), pp. 13-25 (September 1956); also private communication, C. C. Byers (January 1956). 


\section{REF ERENCES（Continued）}

(39) Los Alamos Scientific Laboratory of the University of California, An Enriched Homogeneous Nuclear Reactor, RSI 22, 489-499 (July 1951); a 1so L. D. P. King, Wate $\bar{r}$ Boilers, LA-1034 (December 1947).

(40) R. E. Carter and J. C. Hinton, Water Tamper Measurements, LA-241 (March 1945).

(41) J. C. Allred, P. J. Bendt and, R. E. Peterson, Critical Measurements on $\mathrm{UO}_{3}-\mathrm{H}_{3} \mathrm{PO}_{4}$ Solutions, Nuclear sci. and Eng. 4, 498-500 (1958).

(42) Private communication, J. C. Allred, P. J. Bendt, H. C. Paxton and R. E. Peterson (April 1953).

(43) G. A. Linenberger, J. D. Orndoff and H. C. Paxton, Enriched-Uranium Hydride Critical Assemblies, Nuclear Sci. and Eng . T, 44-57 (1960).

(44) Private communication, M. G. Holloway and C. P. Baker (December 1947).

(45) H. C. Paxton and G. A. Linenberger, Polythene-25 Critical Assembly and Neutron Distribution studies, LA-749 (September 1949).

(46) J. C. Hoogterp, Critical Masses of Oralloy Lattices Immersed in Water, LA-2026 (November 1955).

(47) G. A. Jarvis, G. A. Linenberger, J. D. Orndof $f$ and H. C. Paxton, Two Plutonium-Metal Critical Assemblies, Nuclear Sc1. and Eng. 8, 525-531 (1960).

(48) E. A. Plassmann and D. P. Wood, Critical Reflector Thicknesses for Spherical U233 and Pu239 Systems, Nuclear Sci. and Eng . 8, 615-620 (1960).

(49) Private communication, D. P. Wood and C. C. Byers (December 1960).

(50) Private communication, H. C. Paxton (October 1951).

(51) D. P. Wood, C. C. Byers and L. C. Osborn, Critical Masses of Plutonium Diluted with Other Metals, Nuclear Sci. and Eng. 8, 578-587 (1960). 


\section{REFERENCES（Continued）}

(52) Private communication, G. E. Hansen (October 1963).

(53) Private communication, H. C. Paxton, G. E. Hansen, D. P. Wood and E. A. Plassmann (May 1960).

(54) D. M. Barton, W. Bernard and G. E. Hansen, Critical Masses of Composites of $\mathrm{Oy}$ and Pu-239-240 in Flattop Geonietry, LAMS-2489 (December 1960).

(55) G. E. Hansen and H. C. Paxton, Reevaluated Critical Specifications of Some Los Alamos Fast-Neutron Systems, LA-4208 (September 1969).

(56) L. J. Sapir, H. H. Helmick and J. D. Orndoff, BIG TEN, a 10\% Enriched Uranium Critical Assembly: Kinetic Studies, ANS Trans., 15, No. 1, 312 (June 1972); also private communication, G. E. Hansen and H. C. Paxton (December 1974).

(57) Private communication, G. E. Hansen (November 1966).

(58) J. C. Hoogterp, G. E. Hansen and H. C. Paxton, Bare

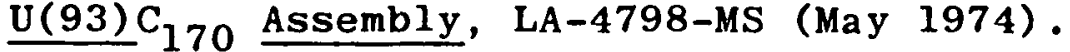

(59) G. A. Jarvis and C. B. Mills, Critical Mass Reduction, LA-3651 (March 1967).

(60) H. C. Paxton, G. A. Jarvis and C. C. Byers, ReflectorModerated Critical Assemblies, LA-5963 (July 1975).

(61) Private communication; E. A. Plassmann (October 1975). 